\title{
A flavor kit for BSM models
}

\author{
Werner Porod ${ }^{1}$, Florian Staub ${ }^{2, a}$, Avelino Vicente ${ }^{3}$ \\ ${ }^{1}$ Institut für Theoretische Physik und Astronomie, Universität Würzburg, 97074 Würzburg, Germany \\ ${ }^{2}$ BCTP and Physikalisches Institut der Universität Bonn, Nußallee 12, 53115 Bonn, Germany \\ ${ }^{3}$ IFPA, Dep. AGO, Université de Liège, Bat B5, Sart-Tilman, 4000 Liège 1, Belgium
}

Received: 12 May 2014 / Accepted: 19 July 2014 / Published online: 28 August 2014

(C) The Author(s) 2014. This article is published with open access at Springerlink.com

\begin{abstract}
We present a new kit for the study of flavor observables in models beyond the standard model. The setup is based on the public codes SARAH and SPheno and allows for an easy implementation of new observables. The Wilson coefficients of the corresponding operators in the effective lagrangian are computed by SPheno modules written by SARAH. New operators can also be added by the user in a modular way. For this purpose a handy Mathematica package called the PreSARAH has been developed. This uses FeynArts and FormCalc to derive generic form factors at tree- and 1-loop levels and to generate the necessary input files for SARAH. This framework has been used to implement $\mathrm{BR}\left(\ell_{\alpha} \rightarrow \ell_{\beta} \gamma\right), \mathrm{BR}\left(\ell_{\alpha} \rightarrow 3 \ell_{\beta}\right), \mathrm{CR}(\mu-e, A), \mathrm{BR}(\tau \rightarrow$ $P \ell), \operatorname{BR}\left(h \rightarrow \ell_{\alpha} \ell_{\beta}\right), \operatorname{BR}\left(Z \rightarrow \ell_{\alpha} \ell_{\beta}\right), \operatorname{BR}\left(B_{s, d}^{0} \rightarrow \ell \bar{\ell}\right)$, $\mathrm{BR}\left(\bar{B} \rightarrow X_{s} \gamma\right), \mathrm{BR}\left(\bar{B} \rightarrow X_{s} \ell \bar{\ell}\right), \operatorname{BR}\left(\bar{B} \rightarrow X_{d, s} \nu \bar{v}\right)$, $\operatorname{BR}\left(K^{+} \rightarrow \pi^{+} v \bar{v}\right), \operatorname{BR}\left(K_{L} \rightarrow \pi^{0} v \bar{v}\right), \Delta M_{B_{s}, B_{d}}, \Delta M_{K}$, $\varepsilon_{K}, \mathrm{BR}(B \rightarrow K \mu \bar{\mu}), \mathrm{BR}(B \rightarrow \ell v), \mathrm{BR}\left(D_{s} \rightarrow \ell v\right)$ and $\mathrm{BR}(K \rightarrow \ell \nu)$ in SARAH. Predictions for these observables can now be obtained in a wide range of SUSY and non-SUSY models. Finally, the user can use the same approach to easily compute additional observables.
\end{abstract}

\section{Contents}

1 Introduction ................. 2

2 General strategy: calculation of flavor observables in a nutshell . . . . . . . . . . . . 2

3 Setup .................. 4

3.1 Flavorkit: usage and goals ...... . 4

3.2 Download and installation ........ 5

3.3 Basic usage . . . . . . . . . . . . 5

3.4 Limitations . . . . . . . . . . . 6

4 Advanced usage I: implementation of new observables using existing operators . . . . . . . . . .

a e-mail: fnstaub@th.physik.uni-bonn.de
5 Advanced usage II: implementation of new operators 9

5.1 Introduction . . . . . . . . . . . . 9 9

5.2 Input for PreSARAH . . . . . . . . . . 9

5.3 Operators with massless gauge bosons . . . . 12

5.4 Combination and normalization of operators . 13

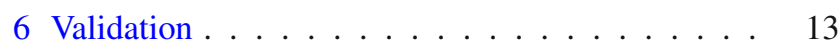

7 Conclusion . . . . . . . . . . . . . . 16

Appendix A: Lagrangian . . . . . . . . . . . . 17

Appendix B: Operators available by default in the SPheno output of SARAH . . . . . . . . 18

B.1 2-Fermion-1-Boson operators . . . . . . 18

B.2 4-Fermion operators . . . . . . . . . 21

Appendix C: Application: flavor observables implemented in SARAH . . . . . . . . . . . . . . 24

C.1 Lepton flavor observables . . . . . . . . . . . 24

C.1.1 $\ell_{\alpha} \rightarrow \ell_{\beta} \gamma$............ 24

C.1.2 $\ell_{\alpha} \rightarrow 3 \ell_{\beta} \ldots . . . . . . .24$

C.1.3 Coherent $\boldsymbol{\mu}-\boldsymbol{e}$ conversion in nuclei . . 26

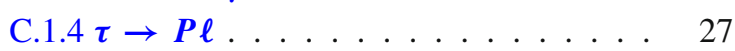

C.1.5h $\rightarrow \ell_{\alpha} \ell_{\beta} \ldots \ldots . . . . . . .30$

C.1.6Z $\rightarrow \ell_{\alpha} \ell_{\beta}$........... 30

C.2 Quark flavor observables . . . . . . . . . 31

C.3 $\boldsymbol{B}_{\boldsymbol{s}, \boldsymbol{d}}^{\mathbf{0}} \rightarrow \ell^{+} \ell^{-} \ldots \ldots . \ldots . . . . . . .31$

C. $4 \bar{B} \rightarrow X_{s} \gamma \ldots \ldots . \ldots \ldots 33$

C.5 $\bar{B} \rightarrow X_{s} \ell^{+} \ell^{-} \ldots \ldots . \ldots . \ldots 34$

C.6 $\boldsymbol{B}^{+} \rightarrow \mathrm{K}^{+} \ell^{+} \ell^{-} \ldots \ldots . \ldots . . . . .36$

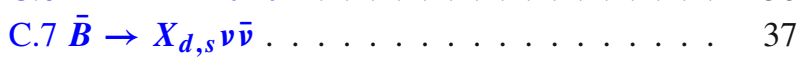

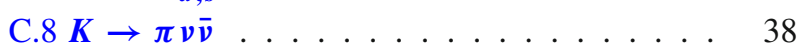

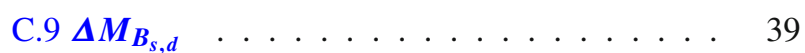

C.10 $\Delta \boldsymbol{M}_{\boldsymbol{K}}$ and $\boldsymbol{\varepsilon}_{\boldsymbol{K}} \ldots \ldots \ldots$. . . . . . . . . 40

C.11P $\rightarrow$ lv.............. 42

Appendix D: Models . . . . . . . . . . . 43

D.1 Supersymmetric models . . . . . . . . . 43

D.2 Non-supersymmetric models . . . . . . . . 44

References ............... . 44 


\section{Introduction}

With the exploration of the terascale, particle physics has entered a new era. On the one hand, the discovery of a Higgs boson at the LHC $[1,2]$ seemingly completed the Standard Model (SM) of particle physics, even though there is still quite some room for deviations from the SM predictions. The observed mass of about $125 \mathrm{GeV}$ in combination with a top quark mass of $173.34 \mathrm{GeV}$ [3] implies within the SM that we potentially live in a meta-stable vacuum [4]. This, together with other observations, like the dark matter relic density or the unification of gauge forces, indicates that there is physics beyond the SM (BSM). Although no sign of new physics has been found so far at the LHC, colliders are not the only places where one can search for new physics. Low energy experiments focused on flavor observables can also play a major role in this regard, since new particles leave their traces via quantum effects in flavor violating processes such as $b \rightarrow s \gamma, B_{s} \rightarrow \mu^{+} \mu^{-}$or $\mu \rightarrow e \gamma$. In the last few years there has been a tremendous progress in this field, both on the experimental as well as on the theoretical side. In particular, observables from the Kaon- and B-meson sectors, rare lepton decays and electric dipole moments have put stringent bounds on new flavor mixing parameters and/or additional phases in models beyond the SM.

There are several public tools on the market which predict the rates of several flavor observables: superiso [5-7], SUSY_Flavor [8,9], NMSSM-Tools [10], Micromegas [11-15], SuperBSG [16], SupeLFV [17], SuseFlav [18], IsaJet with IsaTools [19-24] or SPheno [25,26]. However, all of these codes have in common that they are only valid in the Two-Higgs-doublet model or in the MSSM or simple extensions of it (NMSSM, bilinear R-parity violation). In addition, none of these tools can be easily extended by the user to calculate additional observables. This has made flavor studies beyond the SM a cumbersome task. The situation has changed with the development of SARAH [27-31]. This Mathematica package can be used to generate modules for SPheno, which then can calculate flavor observables at the 1-loop level in a wide range of supersymmetric and non-supersymmetric models [32-34]. However, so far all the information about the underlying Wilson coefficients ${ }^{1}$ for the operators triggering the flavor violation as well as the calculation of the flavor observables had been hardcoded in SARAH. Therefore, it was also very difficult for the user to extend the list of calculated observables. The implementation of new operators was even more difficult.

We present a new kit for the study of flavor observables beyond the standard model. In contrast to previous flavor

\footnotetext{
${ }^{1}$ Sometimes the Wilson coefficients are also referred to as form factors. We will nevertheless stick to the name Wilson coefficients in the following, also for lepton flavor violating processes.
}

codes, Flavorkit is not restricted to a single model, but can be used to obtain predictions for flavor observables in a wide range of models (SUSY and non-SUSY). FlavorKit can be used in two different ways. The basic usage of Flavorkit allows for the computation of a large number of lepton and quark flavor observables, using generic analytical expressions for the Wilson coefficients of the relevant operators. The setup is based on the public codes SARAH and SPheno, and thus allows for the analytical and numerical computation of the observables in the model defined by the user. If necessary, the user can also go beyond the basic usage and define his own operators and/or observables. For this purpose, a Mathematica package called PreSARAH has been developed. This tool uses FeynArts/FormCalc [35-40] to compute generic expressions for the required Wilson coefficients at the tree- and 1-loop levels. Similarly, the user can easily implement new observables. With all these tools properly combined, the user can obtain analytical and numerical results for the observables of his interest in the model of his choice. To calculate new flavor observables with SPheno for a given model the user only needs the definition of the operators and the corresponding expressions for the observables as well as the model file for SARAH. All necessary calculations are done automatically. We have used this setup to implement $\mathrm{BR}\left(\ell_{\alpha} \rightarrow \ell_{\beta} \gamma\right), \mathrm{BR}\left(\ell_{\alpha} \rightarrow 3 \ell_{\beta}\right), \mathrm{CR}(\mu-e, A), \operatorname{BR}(\tau \rightarrow$ $P \ell), \operatorname{BR}\left(h \rightarrow \ell_{\alpha} \ell_{\beta}\right), \operatorname{BR}\left(Z \rightarrow \ell_{\alpha} \ell_{\beta}\right), \operatorname{BR}\left(B_{s, d}^{0} \rightarrow \ell \bar{\ell}\right)$, $\mathrm{BR}\left(\bar{B} \rightarrow X_{s} \gamma\right), \operatorname{BR}\left(\bar{B} \rightarrow X_{s} \ell \bar{\ell}\right), \operatorname{BR}\left(\bar{B} \rightarrow X_{d, s} v \bar{v}\right)$, $\mathrm{BR}\left(K^{+} \rightarrow \pi^{+} \nu \bar{v}\right), \mathrm{BR}\left(K_{L} \rightarrow \pi^{0} v \bar{v}\right), \Delta M_{B_{s}, B_{d}}, \Delta M_{K}$, $\varepsilon_{K}, \operatorname{BR}(B \rightarrow K \mu \bar{\mu}), \operatorname{BR}(B \rightarrow \ell v), \operatorname{BR}\left(D_{s} \rightarrow \ell v\right)$ and $\mathrm{BR}(K \rightarrow \ell v)$ in SARAH.

This manual is structured as follows: in the next section we give a brief introduction into the calculation of flavor observables focusing on the main steps that one has to follow. Then we present Flavorkit, our setup to combine FeynArts/FormCalc, SPheno and SARAH in Sect. 3. In Sect. 4 we explain how new observables can be added and in Sect. 5 how the list of operators can be extended by the user. A comparison between Flavorkit and the other public codes is presented in Sect. 6 taking the MSSM as an example before we conclude in Sect. 7. The appendix contains information about the existing operators and how they have been combined to compute the different flavor observables.

\section{General strategy: calculation of flavor observables in a nutshell}

Once we have chosen a BSM model, ${ }^{2}$ our general strategy for the computation of a flavor observable follows these steps:

\footnotetext{
2 The current version of Flavorkit can only handle renormalizable operators at this stage of the computation.
} 
- Step 1: We first consider an effective Lagrangian that includes the operators relevant for the flavor observable of our interest,

$$
\mathcal{L}_{\text {eff }}=\sum_{i} C_{i} \mathcal{O}_{i}
$$

This Lagrangian consists of a list of (usually) higherdimensional operators $\mathcal{O}_{i}$. The Wilson coefficients $C_{i}$ can be induced either at tree or at higher loop levels and include both the SM and the BSM contributions $\left(C_{i}=\right.$ $C_{i}^{\mathrm{SM}}+C_{i}^{\mathrm{BSM}}$ ). They encode the physics of our model.

- Step 2: The Wilson coefficients are computed diagrammatically, taking into account all possible tree-level and 1-loop topologies leading to the $\mathcal{O}_{i}$ operators. ${ }^{3}$

- Step 3: The results for the Wilson coefficients are plugged in a general expression for the observable and a final result is obtained.

The user has to make a choice in step 1 . The list of operators in the effective Lagrangian can be restricted to the most relevant ones or include additional operators beyond the leading contribution, depending on the required level of precision. Usually, the complete set of renormalizable operators contributing to the observable of interest is considered, although in some well motivated cases one may decide to concentrate on a smaller subset of operators. This freedom is not present in step 2. Once the list of operators has been arranged, the computation of the corresponding $C_{i}$ coefficients follows from the consideration of all topologies (penguin diagrams, box diagrams, ...) leading to the $\mathcal{O}_{i}$ operators. This is the most complicated and model dependent step, since it demands a full knowledge of all masses and vertices in the model under study. Furthermore, it may be necessary to compute the coefficients at an energy scale and then obtain, by means of their renormalization group running, their values at a different scale. Finally, step 3 is usually quite straightforward since, like step 1, is model independent. In fact, the literature contains general expressions for most flavor observables, thus facilitating the final step. However, one should be aware that the formulas given in the literature assume that certain operators contribute only sub-dominantly and, thus, omit the corresponding contributions. This is in general justified for the SM but not in a general BSM model. In particular, this is the case for processes involving external neutrinos, which are often assumed to be purely left-handed, making the operators associated to their right-handed components to be neglected.

\footnotetext{
${ }^{3}$ In principle, one can go beyond the 1-loop level, although in our case we will restrict our computation to the addition of a few NLO corrections.
}

We will exemplify our strategy using a simple example: $\mathrm{BR}(\mu \rightarrow e \gamma)$ in the Standard Model extended by righthanded neutrinos and Dirac neutrino masses. The starting point is, as explained above, to choose the relevant operators. In this case, it is well known that only dipole interactions can contribute to to the radiative decay $\ell_{\alpha} \rightarrow \ell_{\beta} \gamma$ at leading order. ${ }^{4}$ Therefore, the relevant operators are contained in the $\ell-\ell-\gamma$ dipole interaction Lagrangian. This is in general given by

$\mathcal{L}_{\ell \ell \gamma}^{\text {dipole }}=$ ie $m_{\ell_{\alpha}} \bar{\ell}_{\beta} \sigma^{\mu \nu} q_{\nu}\left(K_{2}^{L} P_{L}+K_{2}^{R} P_{R}\right) \ell_{\alpha} A_{\mu}+$ h.c.

Here $e$ is the electric charge, $q$ the photon momentum, $P_{L, R}=\frac{1}{2}\left(1 \mp \gamma_{5}\right)$ are the usual chirality projectors and $\ell_{\alpha, \beta}$ denote the lepton flavors. This concludes step 1 .

The information about the underlying model is encoded in the coefficients $K_{2}^{L, R}$. In the next step, these coefficients have to be calculated by summing up all Feynman diagrams contributing at a given loop level. Expressions for these coefficients for many different models are available in the literature. In the SM only neutrino loops contribute and one finds [41]

$$
\begin{aligned}
K_{2}^{L} & =\frac{G_{F}}{2 \sqrt{2} \pi^{2}} m_{\mu} \sum_{i} \lambda_{i \mu} \lambda_{i e}^{*}\left(F_{1}+F_{2}\right) \\
K_{2}^{R} & =\frac{G_{F}}{2 \sqrt{2} \pi^{2}} m_{e} \sum_{i} \lambda_{i \mu} \lambda_{i e}^{*}\left(F_{1}-F_{2}\right)
\end{aligned}
$$

Here, $\lambda_{i j}$ denote the entries of the Pontecorvo-MakiNakagawa-Sakata matrix and $F_{1}$ and $F_{2}$ are loop functions. One finds approximately $F_{1} \simeq-\frac{1}{4}\left(\frac{m_{v}}{m_{W}}\right)^{2}$ and $F_{2} \simeq 0$. Finally, we just need to proceed to the last step, the computation of the observable. After computing the Wilson coefficients $K_{2}^{L, R}$ it is easy to relate them to $\operatorname{BR}(\mu \rightarrow e \gamma)$ by using [42]

$\Gamma\left(\ell_{\alpha} \rightarrow \ell_{\beta} \gamma\right)=\frac{\alpha m_{\ell_{\alpha}}^{5}}{4}\left(\left|K_{2}^{L}\right|^{2}+\left|K_{2}^{R}\right|^{2}\right)$,

This expression holds for all models. With this final step, the computation concludes.

As we have seen, the main task to get a prediction for $\operatorname{BR}(\mu \rightarrow e \gamma)$ in a new model is to calculate $K_{2}^{L, R}$. However, this demands the knowledge of all masses and vertices involved. Moreover, in most cases a numerical evaluation of the resulting loop integrals is also welcome. Therefore, even for a simple process like $\mu \rightarrow e \gamma$, a computation from scratch in a new model can be a hard work. In order to solve this practical problem, we are going to present here a fully automatized way to calculate a wide range of flavor observables for several classes of models.

\footnotetext{
${ }^{4}$ At next to leading order, one would also have to consider operators like $\bar{\mu} \gamma_{\nu} e \bar{q} \gamma^{v} q$, to be combined with a $q-q-\gamma$ dipole interaction.
} 


\section{Setup}

\subsection{Flavorkit: usage and goals}

As we have seen, the calculation of flavor observables in a specific model is a very demanding task. A detailed knowledge about the model is required, including

1. expressions for all involved masses and vertices

2. optionally, renormalization group equations to get the running parameters at the considered scale

3. expressions to calculate the operators

4. formulae to obtain the observables from the operators.

Nearly all codes devoted to flavor physics have those pieces hardcoded, and they are only valid for a few specific models. ${ }^{5}$ The only exception is SPheno, thanks to its extendability with new modules for additional models. These modules are generated by the Mathematica package SARAH and provide all necessary information about the calculation of the (loop corrected) mass spectrum, the vertices and the 2-loop RGEs. These expressions, derived from fundamental principles for any (renormalizable) model, contain all the information required for the computation of flavor observables. In fact, SARAH also provides Fortran code for a set of flavor observables. For this output, generic expressions of the necessary Wilson coefficients have been included. These are matched to the model chosen by the user and related to the observables by the standard formulae available in the literature. However, it was hardly possible for the user to extend the list of observables or operators included in SARAH without a profound knowledge of either the corresponding Mathematica or Fortran code.

We present a new setup to fill this gap in SARAH: Flavorkit. As discussed in Sect. 2, the critical step in the computation of a flavor observable is the derivation of analytical expressions for the Wilson coefficients of the relevant operators. This step, being model dependent, requires information about the model spectrum and interactions. However, generic expressions can be derived, later to be matched to the specific spectrum and interaction Lagrangian of a given model. For this purpose, we have created a new Mathematica package called PreSARAH. This package uses the power of FeynArts and FormCalc to calculate generic 1-loop amplitudes, to extract the coefficients of the demanded operators, to translate them into the syntax needed for SARAH and to write the necessary wrapper code. PreSARAH works for any 4-fermion or 2fermion-1-boson operators and will be extended in the future

\footnotetext{
5 Recently, Peng4BSMaLO [43] was made public. This code derives analytical expressions for vector penguins for a model defined in the corresponding FeynArts model file.
}

Table 1 List of flavor violating processes and observables which have been already implemented in Flavorkit. To the left, observables related to lepton flavor, whereas to the right observables associated to quark flavor. See Appendices C.1 and C.2 for the definition of the observables and the relevant references for their calculation

\begin{tabular}{ll}
\hline Lepton flavor & Quark flavor \\
\hline$\ell_{\alpha} \rightarrow \ell_{\beta} \gamma$ & $B_{s, d}^{0} \rightarrow \ell^{+} \ell^{-}$ \\
$\ell_{\alpha} \rightarrow 3 \ell_{\beta}$ & $\bar{B} \rightarrow X_{s} \gamma$ \\
$\mu-e$ conversion in nuclei & $\bar{B} \rightarrow X_{s} \ell^{+} \ell^{-}$ \\
$\tau \rightarrow P \ell$ & $\bar{B} \rightarrow X_{d, s} \nu \bar{v}$ \\
$h \rightarrow \ell_{\alpha} \ell_{\beta}$ & $B \rightarrow K \ell^{+} \ell^{-}$ \\
$Z \rightarrow \ell_{\alpha} \ell_{\beta}$ & $K \rightarrow \pi \nu \bar{v}$ \\
& $\Delta M_{B_{s, d}}$ \\
& $\Delta M_{K}$ and $\varepsilon_{K}$ \\
& $P \rightarrow \ell v$ \\
\hline
\end{tabular}

to include other kinds of operators. The current version already contains a long list of fully implemented operators (see Appendix B). The results for the Wilson coefficients obtained with PreSARAH are then interpreted by SARAH, which adapts the generic expressions to the specific details of the model chosen by the user and uses snippets of Fortran code to calculate flavor observables from the resulting Wilson coefficients. As for the operators, there is a long list of observables already implemented (see Appendices C.1 and C.2). Finally, SARAH can be used to obtain analytical output in LATEX format or to create Fort ran modules for SPheno, thus making possible numerical studies.

Flavorkit can be used in two ways:

- Basic usage: This is the approach to be followed by the user who does not need any operator nor observable beyond what is already implemented in Flavorkit. In this case, Flavorkit reduces to the standard SARAH package. The user can use SARAH to obtain analytical results for the flavor observables and, if he wants to make numerical studies, to produce Fortran modules for SPheno. For the list of implemented operators we refer to Appendix B, whereas the list of implemented observables is given in Table 1.

- Advanced usage: This is the approach to be followed by the user who needs an operator or an observable not included in FlavorKit. In case the user is interested in an operator that is not implemented in Flavorkit, he can define his own operators and get analytical results for their coefficients using PreSARAH. Then the output can be passed to SARAH in order to continue with the basic usage. In case the user is interested in an observable that is not implemented in Flavorkit, this can be easily implemented by the addition of a Fortran file, with a few lines of code relating the observable to the operators in $\mathrm{Fl}$ lavorKit (implemented by default or added by the 


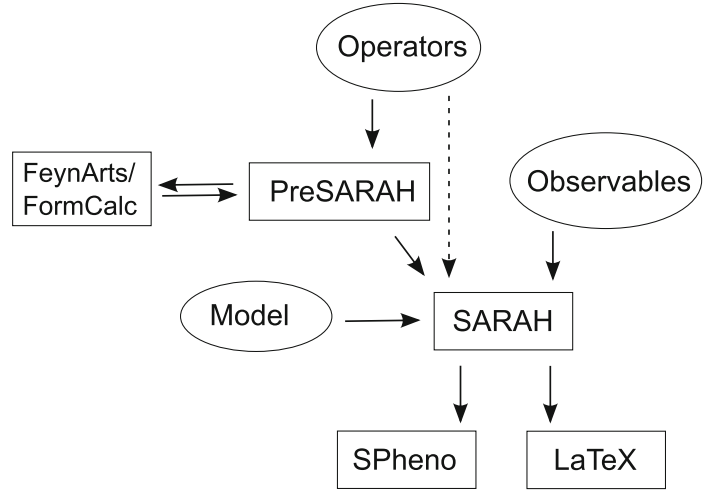

Fig. 1 Schematic way to use Flavorkit: the user can define new operators in PreSARAH, which then calculates the coefficients in a generic form using FeynArts and FormCalc and creates the necessary input files for SARAH. In addition, Fortran code can be provided to relate the Wilson coefficients to specific flavor observables. This information is used by SARAH to generate SPheno code for the numerical calculation of the observables

user). The Fortran files just have to be put together with a short steering file into a specific directory located in the main SARAH directory. Then one can continue with the basic usage.

The combination of PreSARAH together with SARAH and SPheno allows for a modular and precise calculation of flavor observables in a wide range of particles physics models. We have summarized the setup in Fig. 1: the user provides as input SARAH model files for his favorite models or takes one of the models which are already implemented in SARAH (see Appendix D for a list of models available in SARAH). New observables are implemented by providing the necessary Fortran code to SARAH while new operators can be either implemented by hand or by using PreSARAH which then calls FeynArts and FormCalc for the calculation of the necessary diagrams. However, most users will not require to implement new operators or observables. In this case, the user can simply use SARAH in the standard way and (1) derive analytical results for the Wilson coefficients and observables, and (2) generate Fortran modules for SPheno in order to run numerical analysis.

\subsection{Download and installation}

Flavorkit involves several public codes. We proceed to describe how to download and install them.

1. FeynArts/Formcalc

FeynArts and FormCalc can be downloaded from

www.feynarts.de/

It is also possible to use the script FeynInstall, to be found on the same site, for an automatic installation.
2. SARAH and PreSARAH

SARAH can be downloaded from

sarah.hepforge.org/

No installation or compilation is necessary. Both packages just need to be extracted by using tar.

$>$ tar -xf SARAH-4.2.0

$>$ tar -xf PreSARAH-1.0.0

PreSARAH needs the paths to load FeynArts and FormCalc. These have to be provided by the user in the file PreSARAH.ini

1 FeynArtsPackage $=$ "FeynArts/FeynArts.m";

2 FormCalcPackage $=$ "FormCalc/FormCalc.m";

This would work if FeynArts and FormCalc have been installed in the Application directory of the local Mathematica installation. Otherwise, absolute paths should be used, e.g.

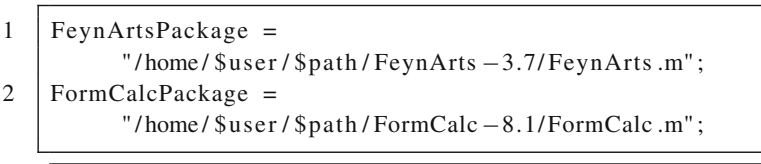

3. SPheno

SPheno can be downloaded from

spheno.hepforge.org/

After extracting the package, make is used for the compilation.

$>$ tar -xf SPheno-3.3.0.tar.gz

$>$ cd SPheno-3.3.0

$>$ make

\subsection{Basic usage}

As explained above, Flavorkit can be used in several ways, depending on the user's needs and interests. The advanced usage, which involves the introduction of new observables and/or the computation of new operators, is explained in detail in Sects. 4 and 5. Here we focus on the basic usage, which just requires the codes SARAH and SPheno.

SARAH can handle the analytical derivation of all the relevant Wilson coefficients in the model defined by the user. The resulting expressions can be then extracted in $\mathrm{LAT}_{\mathrm{E}} \mathrm{X}$ form or used to generate a SPheno module for numerical evaluation. These are the steps to follow in order to use SARAH:

1. Loading SARAH: after starting Ma themat ica, SARAH is loaded via

«SARAH-4.2.0/SARAH.m 
or via

« [\$path] / SARAH-4.2.0/SARAH.m

The first choice works if SARAH has been installed in the Application directory of Mathematica. Otherwise, the absolute path ( [ \$path ] ) to the local SARAH installation must been used.

2. Initialize a model: as example for the initialization of a model in SARAH we consider the NMSSM:

Start [ "NMSSM" ] ;

3. Obtaining the LAT $\mathbf{E}$ X output: the user can get $\mathrm{LAT}_{\mathrm{E}} \mathrm{X}$ output with all the information about the model (including the coefficients for the flavor operators) via

Modeloutput [EWSB] ;

MakeTeX [ ] ;

4. Obtaining the SPheno code: to create the SPheno output the user should run

Makespheno [ ] ;

Thanks to FlavorKit, SARAH can also write LATEX files with the analytical expressions for the Wilson coefficients. These are given individually for each Feynman diagram contributing to the coefficients, and saved in the folder

\section{[\$SARAH]/Output/[\$MODEL]/EWSB/TeX/FlavorKit/}

For the 4-fermion operators the results are divided into separated files for tree-level contributions, penguins contributions and box contributions. The corresponding Feynman diagrams are drawn by using FeynMF [44]. To compile all Feynman diagrams at once and to generate the pdf file, a shell script called MakePDF_[\$OPERATOR]. sh is written as well by SARAH.

In case the user is interested in the numerical evaluation of the flavor observables, a SPheno module must be created as explained above. Once this is done, the resulting Fortran code can be used for the numerical analysis of the model. This can be achieved in the following way:

1. building SPheno: as soon as the SPheno output is finished, open a terminal and enter the root directory of the SPheno installation, and create a new subdirectory, copy the SARAH output to that directory and compile it

$>$ cd [\$SPheno]

$>$ mkdir NMSSM

$>\mathrm{CP}$ [\$SARAH] / Output/NMSSM/EWSB /

SPheno/* NMSSM/

$>$ make Model $=$ NMSSM

2. Running SPheno: After the compilation, a new binary SPhenoNMSSM is created. This file can be executed providing a standard Les Houches input file (SARAH provides an example file, see the SARAH output folder). Finally, SPheno is executed via
$>$./bin/SPhenoNMSSM NMSSM/LesHouches. in. NMSSM

This generates the output file SPheno.spc.NMSSM, which contains the blocks QFVobservables and LFVobservables. In those two blocks, the results for quark and lepton flavor violating observables are given.

Finally, an even easier way to implement new models in SARAH is the butler script provided with the SUSY Toolbox [45]

sarah.hepforge.org/Toolbox/

\subsection{Limitations}

Flavorkit is a tool intended to be as general as possible. For this reason, there are some limitations compared to codes which perform specific calculations in a specific model. Here we list the main limitations of Flavorkit:

- Chiral resummation is not included because of its large model dependence, see e.g. [46] and references therein.

- Even though we have included some of the higher order corrections for the SM part of some observables in a parametric way, 2- or higher loop corrections, calculated in the context of the SM or the MSSM for specific observables, are not considered, see for instance [47-54].

\section{Advanced usage I: implementation of new observables using existing operators}

In order to introduce new observables to the SPheno output of SARAH, the user can add new definitions to the directories

\section{[\$SARAH]/FlavorKit/[\$Type]/Processes/}

[\$Type] is either LFV for lepton flavor violating or QFV for quark flavor violating observables. The definition of the new observables consists of two files

1. A steering file with the extension .m

2. A Fortran body with the extension . f90

The steering file contains the following information:

- NameProcess: a string as name for the set of observables.

- NameObservables: names for the individual observables and numbers which are used to identify them later in the SPheno output. The value is a three dimensional list. The first part of each entry has to be a symbol, the second one an integer and the third one a comment to be printed in the SPheno output file ( $\{$ name1, number 1, comment 1$\}, . .$.$\} ).$ 
- Neededoperators: The operators which are needed to calculate the observables. A list with all operators already implemented in Flavorkit is given in Appendix B. In case the user needs additional operators, this is explained in Sect. 5.

- Body: The name (as string) of the file which contains the Fortran code to calculate the observables from the operators.

For instance, the corresponding file to calculate $\ell_{\alpha} \rightarrow \ell_{\beta} \gamma$ reads

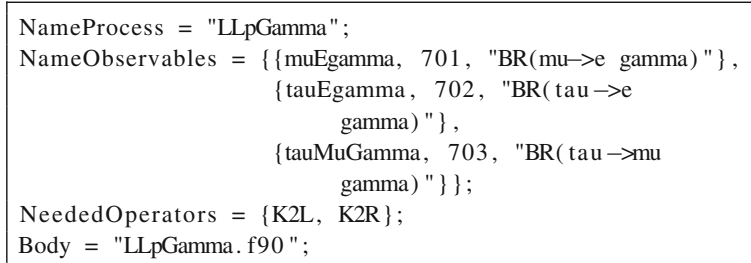

The observables will be saved in the variables muEgamma, tauEgamma, tauMuGamma and will show up in the spectrum file written by SPheno in the block FlavorKitLFV as numbers 701 to 703 .

The file which contains the body to calculate the observables should be standard Fortran 90 code. For our example it reads

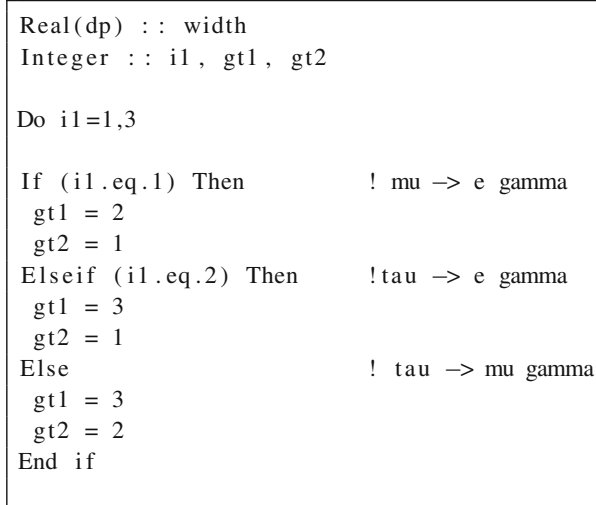

Table 2 List of SM parameters available in Flavorkit. All hadronic observables are calculated at $Q=160 \mathrm{GeV}$

\begin{tabular}{|c|c|c|c|c|c|}
\hline \multicolumn{6}{|l|}{ Real variables } \\
\hline AlphaS_MZ & $\alpha_{S}\left(M_{Z}\right)$ & Alphas_160 & $\alpha_{S}(Q)$ & & \\
\hline sinW2_MZ & $\sin \left(\Theta_{W}\right)^{2}$ at $M_{Z}$ & sinW2_160 & $\sin \left(\Theta_{W}\right)^{2}$ at $Q$ & sinW2 & $\sin \left(\Theta_{W}\right)^{2}$ \\
\hline Alpha_Mz & $\alpha\left(M_{Z}\right)$ & Alpha_160 & $\alpha(Q)$ & Alpha & $\alpha(0)$ \\
\hline MW_MZ & $M_{W}\left(M_{Z}\right)$ & MW_160 & $M_{W}(Q)$ & MW & $M_{W}$ \\
\hline GammaMu & Width $\Gamma_{\mu}$ of $\mu$ & GammaTau & Width $\Gamma_{\tau}$ of $\tau$ & & \\
\hline \multicolumn{6}{|c|}{ Real vectors of length 3} \\
\hline mf_d_160 & $m_{d}(Q)$ & mf_d_MZ & $m_{d}\left(M_{Z}\right)$ & mf_a & $m_{d}$ \\
\hline mf_u_160 & $m_{u}(Q)$ & mf_u_MZ & $m_{u}\left(M_{Z}\right)$ & mf_u & $m_{u}$ \\
\hline mf_1_160 & $m_{l}(Q)$ & $\mathrm{mf}_{-}{ }_{1} \mathrm{MZ}$ & $m_{l}\left(M_{Z}\right)$ & $\mathrm{mf}_{-1}$ & $m_{l}$ \\
\hline \multicolumn{6}{|c|}{ Complex arrays of dimension $3 \times 3$} \\
\hline CKM_MZ & $\mathrm{CKM}$ at $\left(M_{Z}\right)$ & CKM_160 & CKM at $Q$ & $\mathrm{CKM}$ & input \\
\hline
\end{tabular}

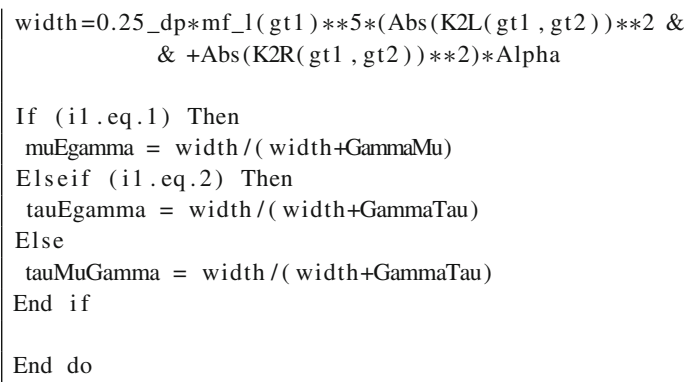

Real (dp) is the SPheno internal definition of double precision variables. Similarly one would have to use Complex (dp) for complex double precision variables when necessary.

Besides the operators, the SM parameters given in Table 2 and the hadronic parameters given in Tables 3 and 4 can be used in the calculations. For instance, we used Alpha for $\alpha(0)$ and mf_l which contains the poles masses of the leptons as well as GammaMu and GammaTau for the total widths of $\mu$ and $\tau$ leptons.

By extending or changing the file hadronic_ parameters.m in the Flavorkit directory, it is possible to add new variables for the mass or life time of mesons. These variables are available globally in the resulting SPheno code. The numerical values for the hadronic parameters can be changed in the Les Houches input file by using the blocks FCONST and FMASS defined in the Flavor Les Houches Accord (FLHA) [55].

It may happen that the calculation of a specific observable has to be adjusted for each model. This is for instance the case when (1) the calculation requires the knowledge of the number of generations of fields, (2) the mass or decay width of a particle, calculated by SPheno, is needed as input, or (3) a rotation matrix of a specific field enters the analytical expressions for the observable. For these situations, a special syntax has been created. It is possible to start a line with @ in the Fortran file. This line will then be parsed by SARAH, and Mathematica commands, as well as SARAH specific 
Table 3 Hadronic parameters used in Flavorkit. These can be changed via FMASS and and FLIFE in the Les Houches input file

\begin{tabular}{|c|c|c|c|c|c|}
\hline Particle & Life time & Default [s] & Mass & Default $[\mathrm{GeV}]$ & PDG number \\
\hline$\pi^{0}$ & tau_pio & $8.52 \cdot 10^{-17}$ & mass_pio & 0.13498 & 111 \\
\hline$\pi^{+}$ & tau_pip & $2.60 \cdot 10^{-8}$ & mass_pip & 0.13957 & 211 \\
\hline$\rho(770)^{0}$ & tau_rho0 & $4.41 \cdot 10^{-24}$ & mass_rho0 & 0.77549 & 113 \\
\hline$D^{0}$ & tau_D0 & $4.10 \cdot 10^{-13}$ & mass_D0 & 1.86486 & 421 \\
\hline$D^{+}$ & tau_Dp & $1.04 \cdot 10^{-12}$ & mass_Dp & 1.86926 & 411 \\
\hline$D_{s}^{+}$ & tau_DSp & $5.00 \cdot 10^{-13}$ & mass_DSp & 1.96849 & 431 \\
\hline$D_{s}^{*+}$ & tau_DSsp & - & mass_DSsp & 2.1123 & 433 \\
\hline$\eta$ & tau_eta & $5.06 \cdot 10^{-19}$ & mass_eta & 0.54785 & 221 \\
\hline$\eta^{\prime}(958)$ & tau_etap & $3.31 \cdot 10^{-21}$ & mass_etap & 0.95778 & 331 \\
\hline$\omega(782)$ & tau_omega & $7.75 \cdot 10^{-23}$ & mass_omega & 0.78265 & 223 \\
\hline$\phi(1020)$ & tau_phi & $1.54 \cdot 10^{-22}$ & mass_phi & 1.01946 & 333 \\
\hline$K_{L}^{0}$ & tau_KL0 & $5.12 \cdot 10^{-8}$ & mass_KL0 & - & 130 \\
\hline$K_{S}^{0}$ & tau_KS0 & $0.90 \cdot 10^{-10}$ & mass_KS0 & - & 310 \\
\hline$K^{0}$ & tau_k0 & - & mass_K0 & 0.49761 & 311 \\
\hline$K^{+}$ & tau_Kp & $1.24 \cdot 10^{-8}$ & mass_Kp & 0.49368 & 321 \\
\hline$B_{d}^{0}$ & tau_B0d & $1.52 \cdot 10^{-12}$ & mass_B0d & 5.27958 & 511 \\
\hline$B_{s}^{0}$ & tau_B0s & $1.50 \cdot 10^{-12}$ & mass_B0s & 5.36677 & 531 \\
\hline$B^{+}$ & tau_Bp & $1.64 \cdot 10^{-12}$ & mass_Bp & 5.27925 & 521 \\
\hline$B^{* 0}$ & tau_B0c & $1.43 \cdot 10^{-23}$ & mass_B0c & 5.3252 & 513 \\
\hline$B^{*+}$ & tau_Bpc & $1.43 \cdot 10^{-23}$ & mass_Bpc & 5.3252 & 523 \\
\hline$B_{c}^{+}$ & tau_Bcp & $4.54 \cdot 10^{-13}$ & mass_Bcp & 6.277 & 541 \\
\hline$K^{* 0}(892)$ & tau_K0c & $1.42 \cdot 10^{-23}$ & mass_K0c & 0.8959 & 313 \\
\hline$K^{*+}(892)$ & tau_Kpc & $1.30 \cdot 10^{-23}$ & mass_Kpc & 0.8917 & 323 \\
\hline$\eta_{c}(1 S)$ & tau_etac & $2.22 \cdot 10^{-23}$ & mass_etac & 2.9810 & 441 \\
\hline$J / \Psi(1 S)$ & tau_JPsi & $7.08 \cdot 10^{-24}$ & mass_JPSi & 3096.92 & 443 \\
\hline$\Upsilon(1 S)$ & tau_Ups & $1.21 \cdot 10^{-23}$ & mass_Ups & 9.4603 & 553 \\
\hline
\end{tabular}

Table 4 Decay constants available in the SPheno output of SARAH. The values can be changed according to the FLHA conventions using the block FCONST in the Les Houches input file

\begin{tabular}{llll}
\hline $\begin{array}{l}\text { Decay } \\
\text { constant }\end{array}$ & Variable & $\begin{array}{l}\text { Default } \\
{[\mathrm{MeV}]}\end{array}$ & FLHA \\
\hline$f_{K}$ & f_k_CONST & 176 & FCONST $[321,1]$ \\
$f_{K^{+}}$ & f_Kp_CONST & 156 & FCONST $[323,1]$ \\
$f_{\pi}$ & f_pi_CONST & 118 & FCONST $[111,1]$ \\
$f_{B_{d}^{0}}$ & f_B0d_CONST & 194 & FCONST $[511,1]$ \\
$f_{B_{s}^{0}}$ & f_B0.C_CONST & 234 & FCONST $[531,1]$ \\
$f_{B^{+}}$ & f_Bp_CONST & 234 & FCONST $[521,1]$ \\
$f_{\eta^{\prime}}$ & f_etap_CONST & 172 & FCONST $[231,1]$ \\
$f_{\rho}$ & f_rho_CONST & 220 & FCONST $[213,1]$ \\
$f_{D^{+}}$ & f_Dp_CONST & 256 & FCONST $[411,1]$ \\
$f_{D_{s}}$ & f_DS_CONST & 248 & FCONST $[431,1]$ \\
\hline
\end{tabular}

commands, can be used. We made use of this functionality in the implementation of $h \rightarrow \ell_{\alpha} \ell_{\beta}$. The lines in hLLp. $f 90$ read

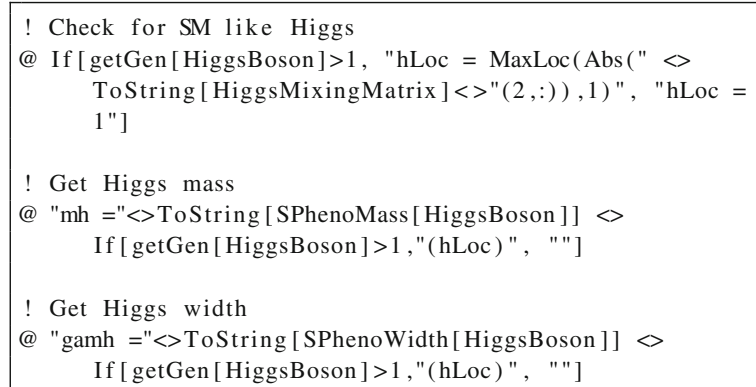

In this implementation we define an integer hLoc that gives the generation index of the SM-like Higgs, to be found among all $\mathrm{CP}$ even scalars. In the first line it is checked if more than one scalar Higgs is present. If this is the case, the hLoc is set to the component which has the largest amount of the up-type Higgs, if not, it is just put to 1 . Of course, this assumes that the electroweak basis in the Higgs sector is always defined as $\left(\phi_{d}, \phi_{u}, \ldots\right)$ as is the case for all models delivered with SARAH. In the second and third lines, the variables mh and gamh are set to the mass and total width of the SM-like Higgs, respectively. For this purpose, the SARAH commands 
Table 5 SARAH commands which can be used in the input file for the calculation of an observable

\begin{tabular}{lc}
\hline x getGen $[\mathrm{x}]$ & $\begin{array}{c}\text { Returns the number of } \\
\text { generations of a particle } \mathrm{x} \\
\text { Returns the dimension of a } \\
\text { variable } \mathrm{x}\end{array}$ \\
Returns the name used for the \\
mass of a particle $\mathrm{x}$ in the \\
SPheno output \\
Returns the name used for the \\
mass squared of a particle $\mathrm{x}$ \\
in the SPheno output \\
SPhenoMassSq[x] & $\begin{array}{c}\text { Returns the name used for the } \\
\text { width of a particle } \mathrm{x} \text { in the } \\
\text { SPheno output }\end{array}$ \\
SPhenoWidth[x] & Name of the mixing matrix for \\
& the CP even Higgs states in a \\
given model & Name of the mixing matrix for \\
the CP odd Higgs states in a & given model
\end{tabular}

SPhenoMass $[\mathrm{x}$ ] and SPhenowidth [x] are used. They return the name of the variable for the mass and width in SPheno and it is checked if these variables are arrays or not. ${ }^{6}$ For the MSSM, the above lines lead to the following code in the SPheno output:

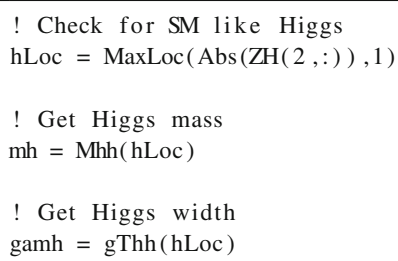

We give in Table 5 the most important SARAH commands which might be useful in this context.

Many more examples are given in Appendix C.1, where we have added all input files for the calculations of flavor observables delivered with SARAH.

\section{Advanced usage II: implementation of new operators}

The user can also implement new operators and obtain analytical expressions for their Wilson coefficients. In this case,

\footnotetext{
6 The user can define in the parameters.m and particles.m file for a given model in SARAH the particles which should be taken to be the CP-even or CP-odd Higgs and the parameter that corresponds to their rotation matrices. This is done by using the Description statements Higgs or Pseudo-Scalar Higgs as well as Scalar-Mixing-Matrix or Pseudo-Scalar-Mixing-Matrix. If the particle or parameter needed to calculate an observable is not present or has not been defined, the observable is skipped in the SPheno output.
}

he will need to use PreSARAH which, with the help of FeynArts and FormCalc, provides generic expressions for the coefficients, later to be adapted to specific models with SARAH.

\subsection{Introduction}

New operators can be implemented by extending the content of the folder

\section{[\$SARAH]/FlavorKit/[\$Type]/Operators/}

In the current version of Flavorkit, 3- and 4-point operators are supported. Each operator is defined by a .m-file. These files contain information about the external particles, the kind of considered diagrams (tree-level, self-energies, penguins, boxes) as well as generic expressions for the coefficients. These expressions, derived from the generic Feynman diagrams contributing to the coefficients, are written in the form of a Mathematica code, which can be used to generate Fortran code.

For the automatization of the underlying calculations we have created an additional Mathematica package called PreSARAH, which can be used to create the files for all 4fermion as well as 2-fermion-1-boson operators. This package creates not only the infrastructure to include the operators in the SPheno output of SARAH but makes also use of FeynArts and FormCalc to calculate the amplitudes and to extract the coefficient of the demanded operators. It takes into account all topologies depicted in Figs. 2, 3, 4, 5 and 6.

\subsection{Input for PreSARAH}

In order to derive the results for the Wilson coefficients, PreSARAH needs an input file with the following information:

- ConsideredProcess: A string which defines the generic type for the process

$$
\begin{aligned}
& \text { - "4Fermion" } \\
& \text { - "2Fermion1Scalar" } \\
& \text { - "2Fermion1Vector" }
\end{aligned}
$$

- NameProcess: A string to uniquely define the process

- Externalfields: The external fields. Possible names are ChargedLepton, Neutrino, DownQuark, UpQuark, ScalarHiggs, PseudoScalar, Zboson, Wboson ${ }^{7}$

\footnotetext{
7 The particles.m file for each model is used to define which particle corresponds to SM states using the Description statement together with Leptons, Neutrinos, Down-Quarks, Up-Quarks, Higgs, Pseudo-Scalar Higgs, Z-Boson, $\mathrm{W}$-Boson. If there is a mixture between the SM particles and other states (like in $R$-parity violating SUSY or in models with additional
} 
Fig. 2 All topologies considered by PreSARAH to calculate the Wilson coefficients of 2-fermion-1-boson operators.

All possible generic combinations of the internal fields are taken into account
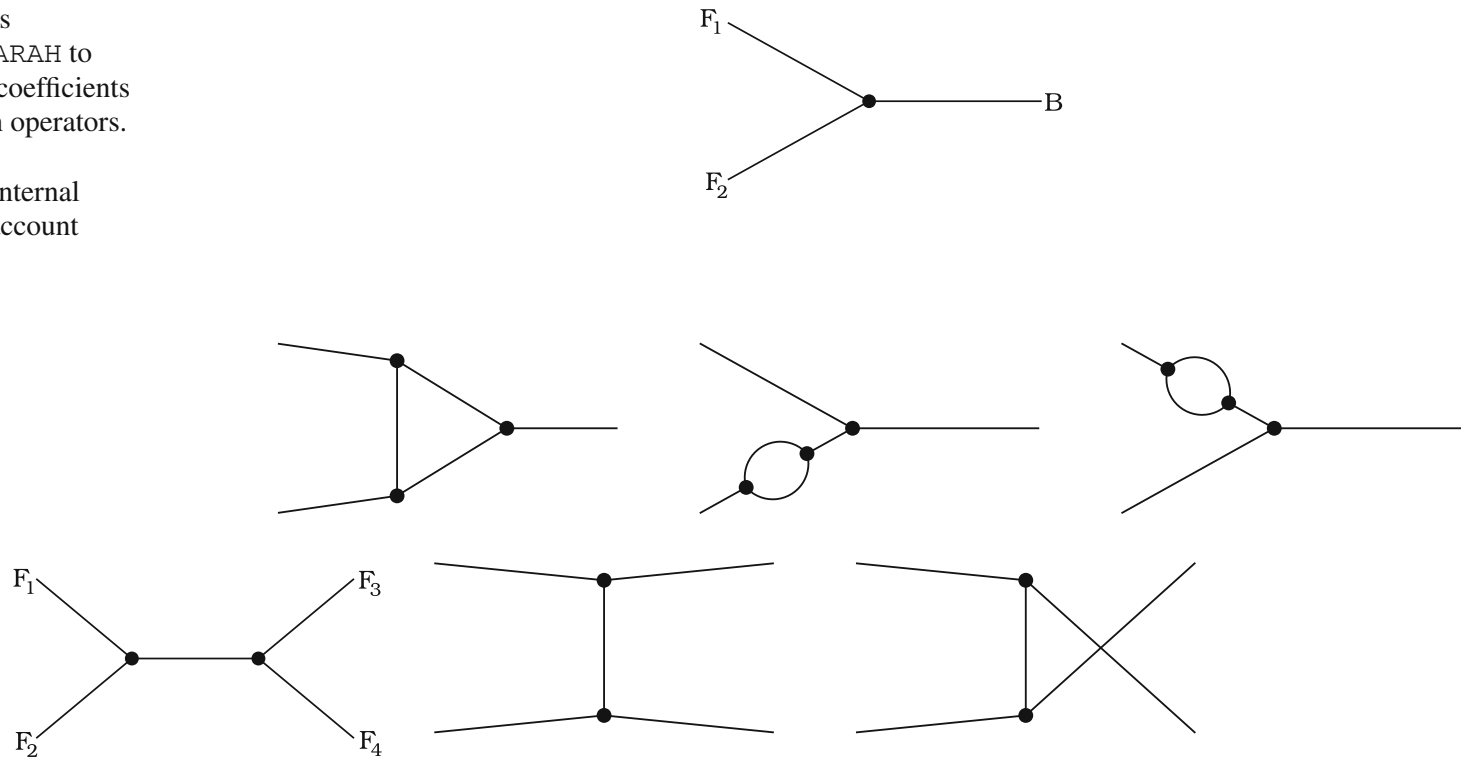

Fig. 3 All tree topologies considered by PreSARAH to calculate the Wilson coefficients of 4-fermion operators. All possible generic combinations of the internal fields are taken into account

Fig. 4 All self-energy

topologies considered by

PreSARAH to calculate the

Wilson coefficients of 4-fermion

operators. All possible generic

combinations of the internal

fields are taken into account
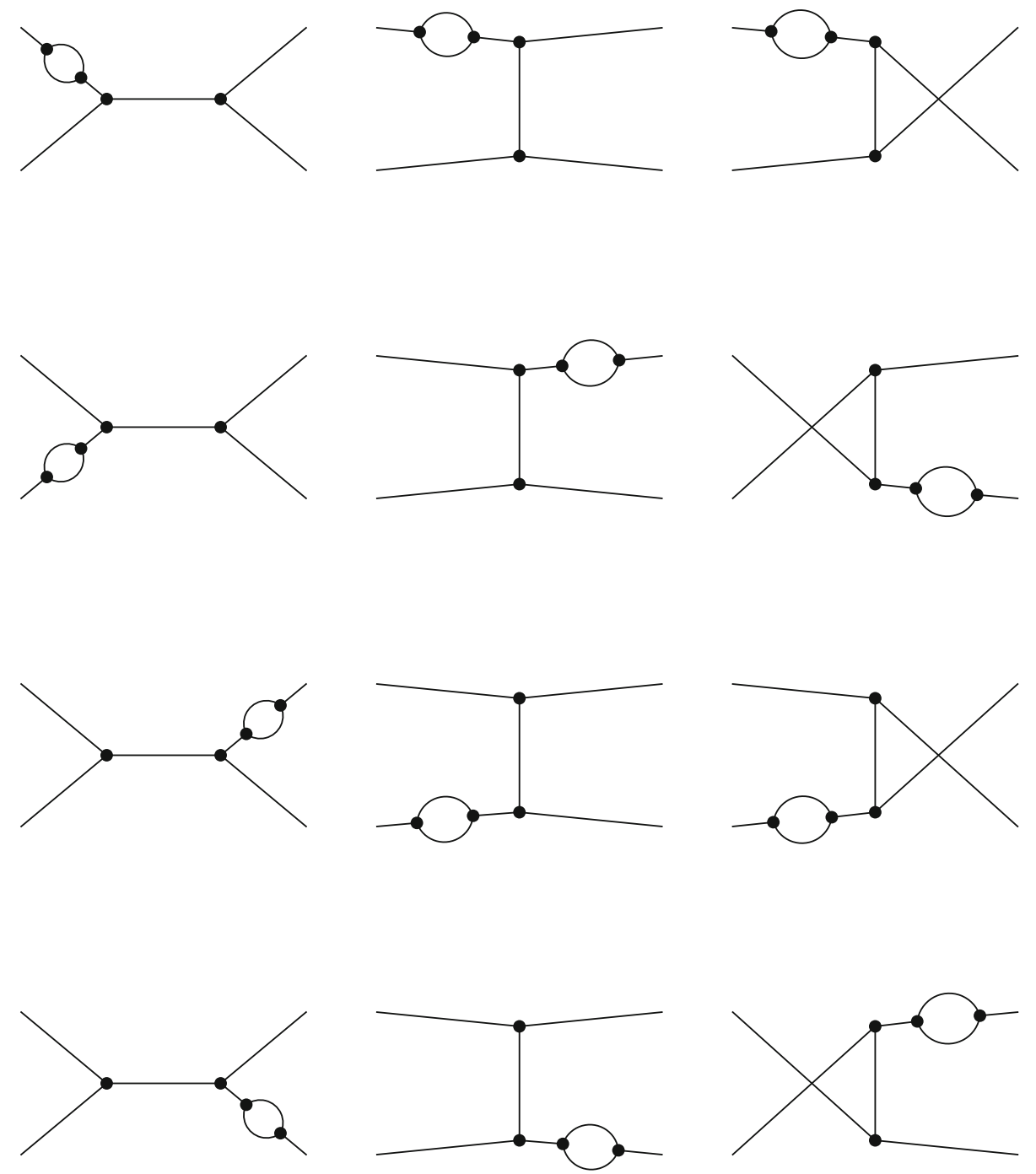
Fig. 5 All penguin topologies considered by PreSARAH to calculate the Wilson coefficients of 4-fermion operators. All possible generic combinations of the internal fields are taken into account
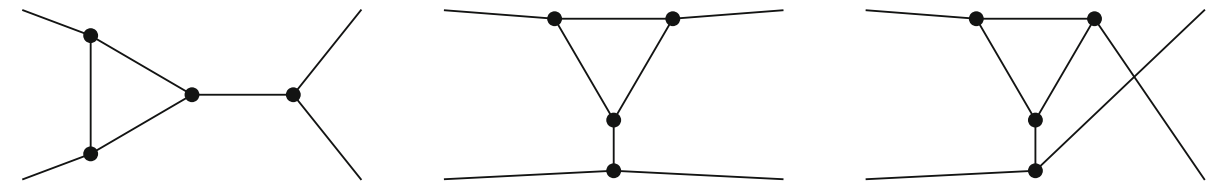

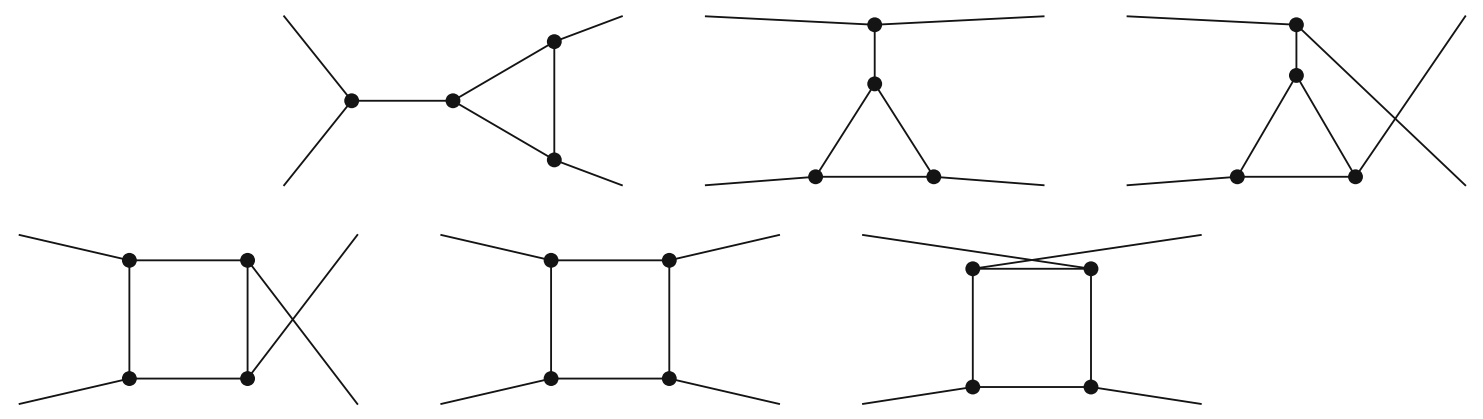

Fig. 6 All box topologies considered by PreSARAH to calculate the Wilson coefficients of 4-fermion operators. All possible generic combinations of the internal fields are taken into account

- FermionorderExternal: the fermion order to apply the Fierz transformation (see the FormCalc manual for more details)

- NeglectMasses: which external masses can be neglected (a list of integers counting the external fields)

- Colorflow: defines the color flow in the case of four quark operators. To contract the colors of external fields, ColorDelta is used, i.e Colorflow = ColorDelta $[1,2] *$ ColorDelta [3, 4] assigns $\left(\bar{q}^{\alpha} \Gamma q_{\alpha}\right)\left(\bar{q}^{\beta} \Gamma^{\prime} q_{\beta}\right)$.

- Alloperators: a list with the definition of the operators. This is a two dimensional list, where the first entry defines the name of the operator and the second one the Lorentz structure. The operators are expressed in the chiral basis and the syntax for Dirac chains in FormCalc is used:

- 6 for $P_{L}=\frac{1}{2}\left(1-\gamma_{5}\right), 7$ for $P_{R}=\frac{1}{2}\left(1-\gamma_{5}\right)$

- Lor [1], Lor [2] for $\gamma_{\mu}, \gamma_{\nu}$

- ec [3] for the helicity of an external gauge boson

- $\mathrm{k}[\mathrm{N}]$ for the momentum of the external particle $\mathrm{N}(\mathrm{N}$ is an integer)

- Pair [A, B] is used to contract Lorentz indices. For instance, Pair [k[1], ec [3] ] stands for $k_{\mu}^{1} \epsilon^{\mu, *}$

- A Dirac chain starting with a negative first entry is taken to be anti-symmetrized.

Footnote 7 continued

vector quarks/leptons) the combined state has to be labeled with those description. Pseudo-Scalar Higgs is in the SM just the neutral Goldstone boson. If an external state is not present in a given model or hasn't been defined as such in the particles.m file the corresponding Wilson coefficients are not calculated by SPheno.
See the FormCalc manual for more details.

To make the definitions more readable, not the full DiracChain object of FeynArts/FormCalc has to be defined: PreSARAH puts everything with the head Op into a Dirac chain using the defined fermion order. For 4 -fermion operators the combination of both operators is written as dot product. For instance Op [6].Op [6] is internally translated into

$$
\begin{aligned}
& \text { DiracChain [Spinor[k[1], MassEx1, -1], 6, } \\
& \text { Spinor[k[2], MassEx2,1]] * } \\
& \text { DiracChain[Spinor[k[3], MassEx3,-1], } \\
& \text { 6, Spinor[k[4], MassEx4,1]] }
\end{aligned}
$$

while Op [6] Pair [ec [3],k[1] becomes

$$
\begin{aligned}
& \text { DiracChain [Spinor [k[1], MassEx1,-1], } \\
& \text { 6, Spinor[k[2], MassEx2,1]] } \\
& \text { Pair[ec[3],k[1] ] }
\end{aligned}
$$

- CombinationGenerations: the combination of external generations for which the operators are calculated by SPheno

- Filters: a list of filters to drop specific diagrams. Possible entries are NoBoxes, NoPenguins, NoTree, NoCrossedDiagrams.

- Filters $=$ \{NoBoxes, NoPenguins $\}$ can be used for processes which are already triggered at tree-level

- Filters $=\{$ NoPenguins $\}$ might be useful for processes which at the 1-loop level are only induced by box diagrams 
- Filters = \{NoCrossedDiagrams $\}$ is used to drop diagrams which only differ by a permutation of the external fields.

For instance, the PreSARAH input to calculate the coefficient of the $(\bar{\ell} \Gamma \ell)\left(\bar{d} \Gamma^{\prime} d\right)$ operator reads

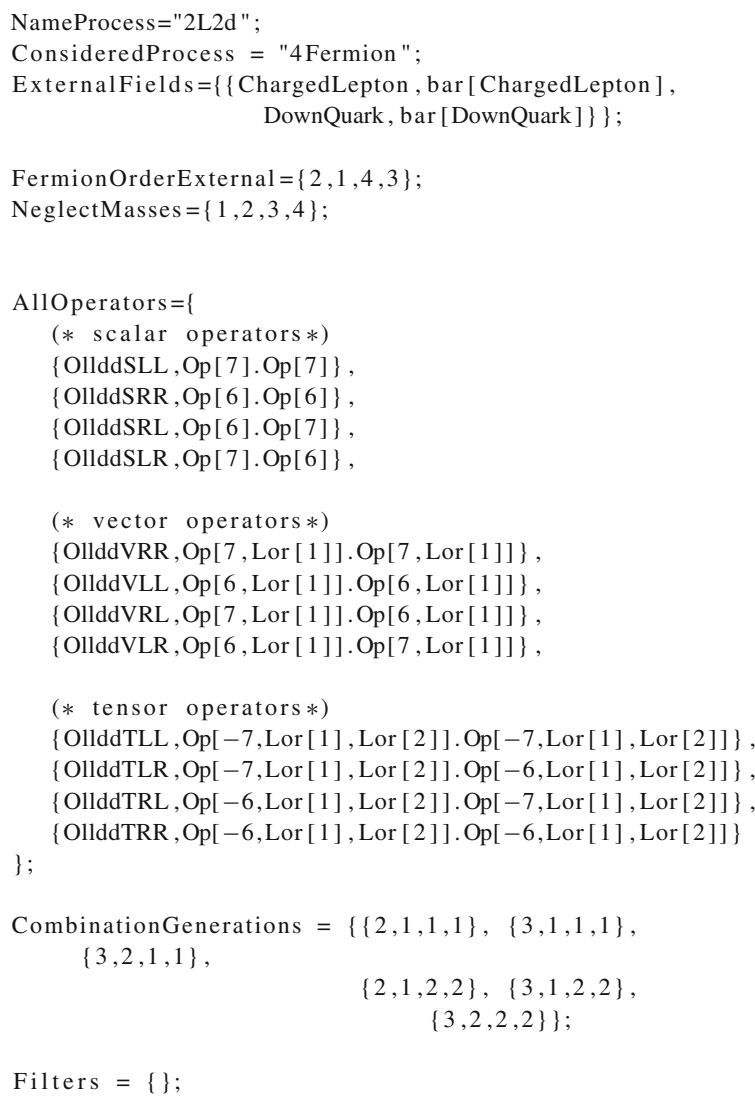

Here, we neglect all external masses in the operators (NeglectMasses $=\{1,2,3,4\}$ ), and the different coefficients of the scalar operators $\left(\bar{\ell} P_{X} \ell\right)\left(\bar{d} P_{Y} d\right)$ are called OllddSXY, the ones for the vector operators $\left(\bar{\ell} P_{X} \gamma_{\mu} \ell\right)$ $\left(\bar{d} P_{Y} \gamma^{\mu} d\right)$ are called OllddVYX and the ones for the tensor operators $\left(\bar{\ell} P_{X} \sigma_{\mu \nu} \ell\right)\left(\bar{d} \sigma^{\mu \nu} P_{Y} d\right)$ OllddTYX, with $\mathrm{X}, \mathrm{Y}=\mathrm{L}, \mathrm{R}$. Notice that FormCalc returns the results in form of $P_{X} \gamma_{\mu}$ while in the literature the order $\gamma_{\mu} P_{X}$ is often used. Finally, SPheno will not calculate all possible combinations of external states, but only some specific cases: $\mu e d d, \tau e d d$, $\tau \mu d d$, $\mu$ ess, $\tau$ ess, $\tau \mu$ ss. ${ }^{8}$

The input file to calculate the coefficients of the $\ell-\ell-Z$ operators $\left(\bar{\ell} \gamma_{\mu} P_{L, R} \ell\right) Z^{\mu}$ and $\left(\bar{\ell} p_{\mu} P_{L, R} \gamma_{\mu} \ell\right) Z^{\mu}$ is

\footnotetext{
${ }^{8}$ Here we used $d$ for the first generation of down-type quarks while in the rest of this manual it is used to summarize all three families.
}

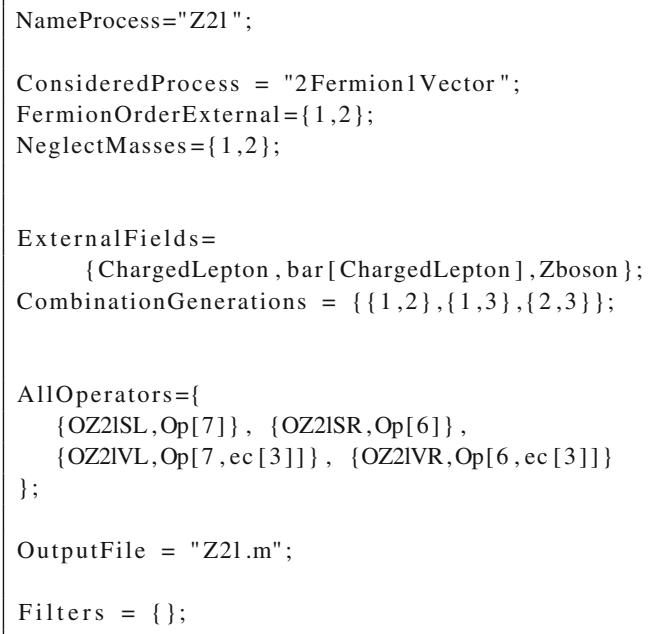

Note that ExternalFields must contain first the involved fermions and the boson at the end. Furthermore, in the case of processes involving scalars one can define

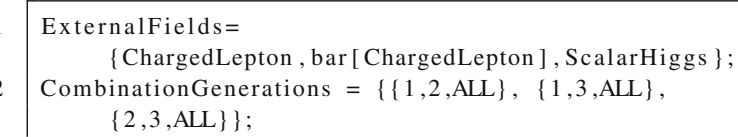

In this case the operators for all Higgs states present in the considered model will be computed.

\subsection{Operators with massless gauge bosons}

We have to add a few more remarks concerning 2-fermion1-boson operators with massless gauge bosons since those are treated in a special way. It is common for these operators to include terms in the amplitude which are proportional to the external masses. Therefore, if one proceeds in the usual way and neglects the external momenta, some inconsistencies would be obtained. For this reason, a special treatment is in order. In PreSARAH, when one uses

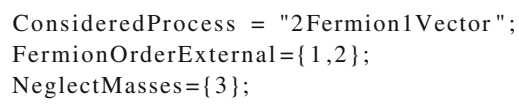

the dependence on the two fermion masses is neglected in the resulting Passarino-Veltman integrals but terms proportional to $m_{f_{1}}$ and $m_{f_{2}}$ are kept. This solves the aforementioned potential inconsistencies.

Furthermore, for the dipole operators, defined by \begin{tabular}{l|l}
1 \\
2
\end{tabular}

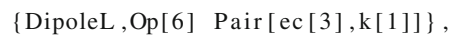

we are using the results obtained by FeynArts and FormCalc and have implemented all special cases for the involved loop integrals $\left(C_{0}, C_{00}, C_{1}, C_{2}, C_{11}, C_{12}, C_{22}\right)$ 
with identical or vanishing internal masses in SPheno. This guarantees the numerical stability of the results. ${ }^{9}$

The monopole operators of the form $q^{2}\left(\bar{f} \gamma_{\mu} f\right) V^{\mu}$ are only non-zero for off-shell external gauge bosons, while PreSARAH always treats all fields as on-shell. Because of this, and to stabilize the numerical evaluation later on, these operators are treated differently to all other operators: the coefficients are not calculated by FeynArts and FormCalc but instead we have included the generic expressions in PreSARAH using a special set of loop functions in SPheno. In these loop functions the resulting PassarinoVeltman integrals are already combined, leading to wellknown expressions in the literature, see [42,56]. They have been cross-checked with the package Peng4BSM@LO [43]. To get the coefficients for the monopole operators, these have to be given always in the form

$\{$ MonopoleL, Op[6, ec[3]] Pair[k[3],k[3]]\},

\{MonopoleR, Op[7, ec [3]] Pair[k[3],k[3]]\}

in the input of PreSARAH.

\subsection{Combination and normalization of operators}

The user can define new operators as combination of existing operators. For this purpose wrapper files containing the definition of the operators can be included in the FlavorKit directories. These files have to begin with ProcessWrapper = True; . This function is also used by PreSARAH in the case of 4-fermion operators: for these operators the contributions stemming from tree-level, box- and penguin- diagrams are saved separately and summed up at the end. Thus, the wrapper code for the 4-lepton operators written by PreSARAH reads

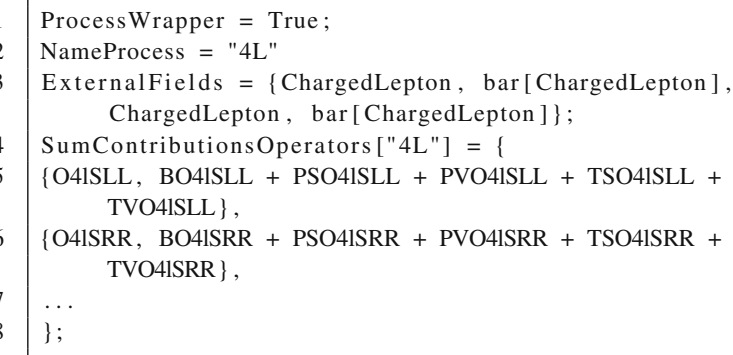

It is also possible to use these wrapper files to change the normalization of the operators. We have made use of this functionality for the operators with external photons and gluons to match the standard definition used in literature: it is common to write these operators as $e m_{f}\left(\bar{f} \sigma_{\mu \nu} f\right) F^{\mu \nu}$, i.e. with the electric coupling (or strong coupling for gluon

\footnotetext{
9 We note that the coefficients for the operators defined above $\left(\bar{f} \gamma_{\mu} f V^{\mu}\right)$ are by a factor of 2 (4) larger than the coefficients of the standard definition for the dipole operators $\bar{f} \sigma_{\mu \nu} P_{L} f q^{\nu} V^{\mu}$ $\left(\bar{f} \sigma_{\mu \nu} P_{L} f F^{\mu \nu}\right)$.
}

operators) and fermion mass factored out. This is done with the files Photon_wrapper.m and Gluon_wrapper.m, included in the FlavorKit directory of SARAH:

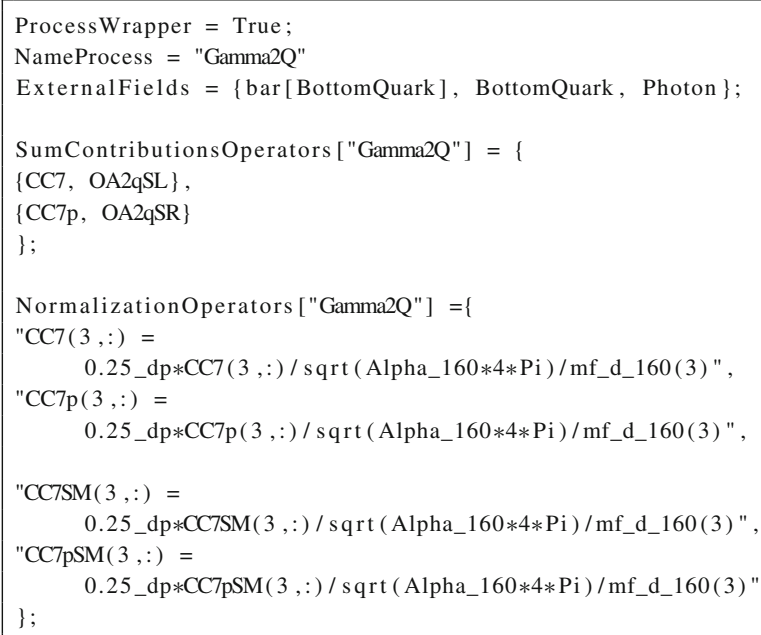

First, the coefficients OA2qSL and OA2 qSR derived with PreSARAH are matched to the new coefficients CC7 and CC7p. The same matching is automatically applied also to the SM coefficients OA2 qSLSM and OA2qSRSM. In a second step, these operators are re-normalized to the standard definition of the Wilson coefficients $C_{7}$ and $C_{7}^{\prime}$. The initial coefficients OA2qSR, OA2qSL are not discarded, but coexist besides CC7, CC7p. Thus, the user can choose in the implementation of the observables which operators are more suitable for his purposes.

\section{Validation}

The validation of the FlavorKit results happened in three steps:

1. Agreement with SM results: we checked that the SM prediction for the observables agree with the results given in literature

2. Independence of scale in loop function: the loop integrals for two and three point functions $\left(B_{i}, C_{i}\right)$ depend on the renormalization scale $Q$. However, this dependence has to drop out for a given process at leading order. We checked numerically that the sum of all diagrams is indeed independent of the choice of $Q$.

3. Comparison with other tools: as we have pointed out in the introduction, there are several public tools which calculate flavor observables mostly in the context of the MSSM. We did a detailed comparison with these tools using the SPheno code produced by SARAH for the MSSM. Some results are presented in the following.

We have compared the Flavorkit results using SARAH 4.2.0 and SPheno3.3.0 with 

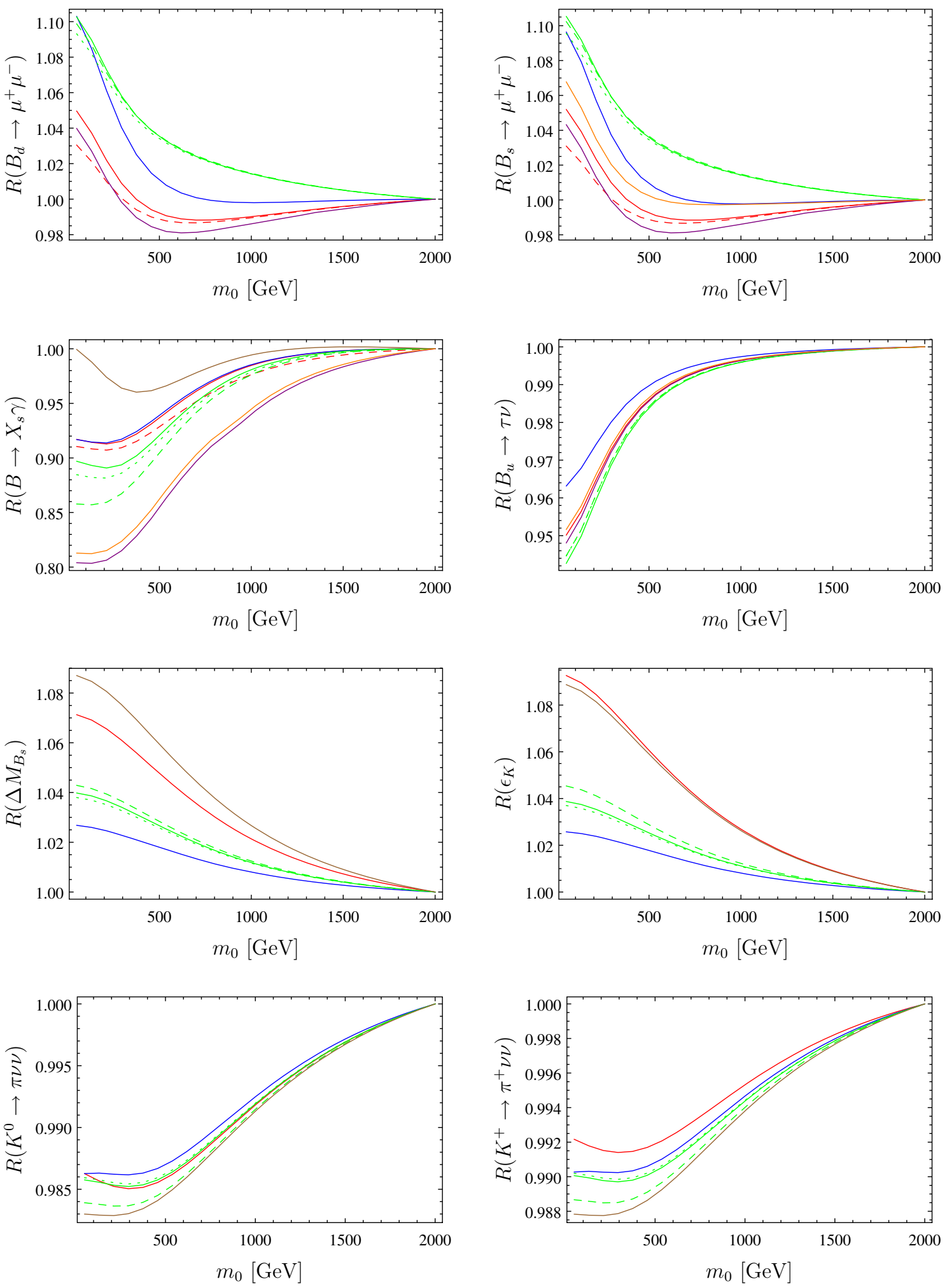

Fig. 7 Comparison of the results for $\operatorname{BR}\left(B_{s, d}^{0} \rightarrow \mu \mu\right), \operatorname{BR}(\bar{B} \rightarrow$ $\left.X_{s} \gamma\right), \operatorname{BR}(B \rightarrow \tau v), \Delta M_{B_{s}}, \varepsilon_{K}, \operatorname{BR}\left(K_{L} \rightarrow \pi^{0} v \bar{v}\right), \operatorname{BR}\left(K^{+} \rightarrow\right.$ $\left.\pi^{+} \nu \bar{\nu}\right)$ as a function of $m_{0}$ using the Flavorkit (red), superiso (purple), SUSY_Flavor 1 (brown), SUSY_Flavor 2 (green),

SPheno (blue), Micromegas (orange) and the old implementation in SARAH (red dashed). The three lines for SUSY_Flavor 2 correspond to different options of the chiral resummation. We used $M_{1 / 2}=200 \mathrm{GeV}, A_{0}=0, \tan \beta=10, \mu>0$ 

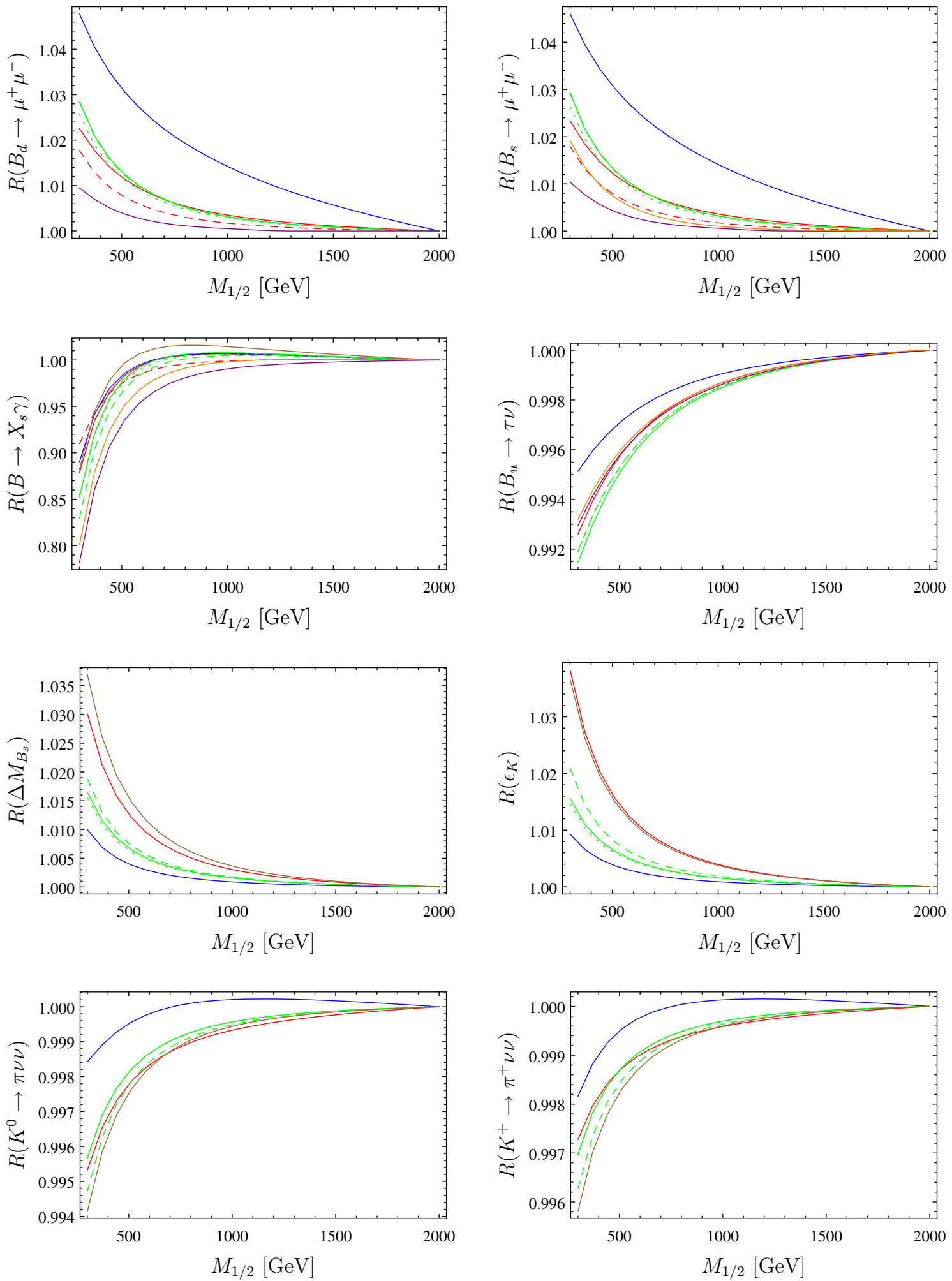

Fig. 8 Comparison of the results for different flavor observables as function of $M_{1 / 2}$. The color code is the same as in Fig. 7. We used $m_{0}=500 \mathrm{GeV}$, $A_{0}=-1000 \mathrm{GeV}, \tan \beta=10, \mu>0$ 

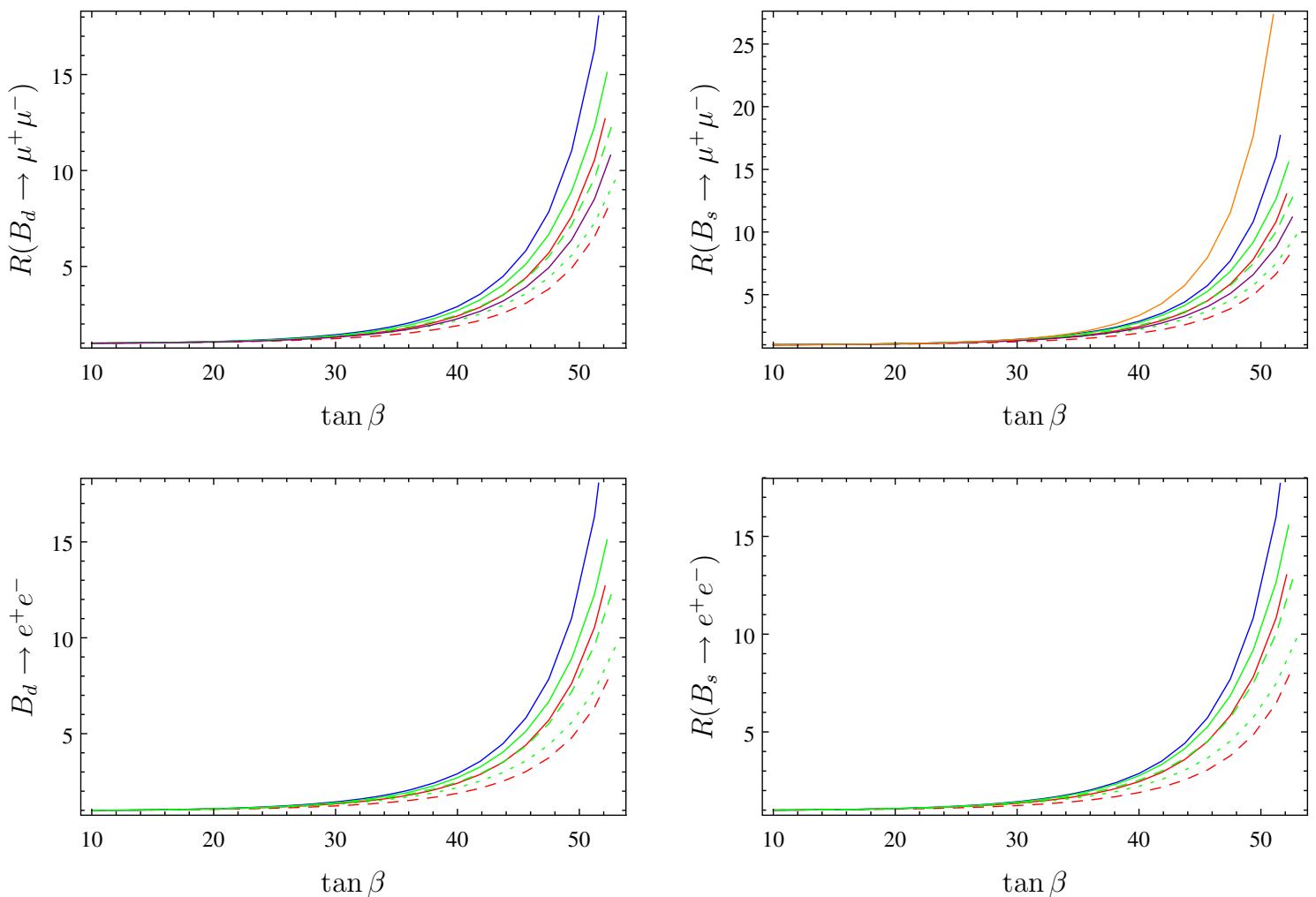

Fig. 9 Comparison of $\mathrm{BR}\left(B_{s, d}^{0} \rightarrow \mu \mu\right)$ (first row) and $\mathrm{BR}\left(B_{s, d}^{0} \rightarrow e e\right)$ (second row) as function of $\tan \beta$. The color code is the same as in Fig. 7 . We used $m_{0}=M_{1 / 2}=500 \mathrm{GeV}, A_{0}=0, \mu>0$

- superiso 3.3

- SUSY_Flavor 1 and 2.1

- MicrOmegas 3.6.7

- SPheno 3.3.0

- a SPheno version produced by SARAH 4.1 .0 without the Flavorkit functionality

Since these codes often use different values for the hadronic parameters and calculate the flavor observables at different loop levels, we are not going to compare the absolute numbers obtained by these tools. Instead, we compare the results normalized to the SM prediction of each code and thus define, for an observable $X$, the ratio

$R(X)=\frac{X^{M S S M}}{X^{S M}}$.

$X^{S M}$ is obtained by taking the value of $X$ calculated by each code in the limit of a very heavy SUSY spectrum. As test case we have used the CMSSM. The dependence of a set of flavor observables as function of $m_{0}$ is shown in Fig. 7 and as function of $M_{1 / 2}$ in Fig. 8 .

We see that all codes show in general the same dependence. However, it is also obvious that the lines are not on top of each other but differences are present. These differences are based on the treatment of the resummation of the bottom Yukawa couplings, the different order at which SM and SUSY contributions are implemented, the different handling of the Weinberg angle, and the different level at which the RGE running is taken into account by the tools. Even if a detailed discussion of the differences of all codes might be very interesting it is, of course, far beyond the scope of this paper and would require a combined effort. The important point is that the results of Flavorkit agree with the codes specialized for the MSSM to the same level as those codes agree among each other. Since the Flavorkit results for all observables are based on the same generic routines it might be even more trustworthy than human implementations of the lengthy expressions needed to calculate these observables because it is less error prone. Of course, known 2-loop corrections for the MSSM which are implemented in other tools are missing.

Finally, it is well known that the process $B_{s, d}^{0} \rightarrow \ell \bar{\ell}$ has a strong dependence on the value of $\tan \beta$. We show in Fig. 9 that this is reproduced by all codes.

\section{Conclusion}

We have presented Flavorkit, a new setup for the calculation of flavor observables for a wide range of BSM models. Generic expressions for the Wilson coefficients are derived with PreSARAH, a Mathematica package that makes use of FeynArts and FormCalc. The output of PreSARAH is 
then passed to SARAH, which generates the Fortran code that allows to calculate numerically the values of these Wilson coefficients with SPheno. The observables are derived by providing the corresponding pieces of Fortran code to SARAH, which incorporates them into the SPheno output. We made use of this code chain to fully implement a large set of important flavor observables in SARAH and SPheno. In fact, due the simplicity of this kit, the user can easily extend the list with his own observables and operators. In conclusion, Flavorkit allows the user to easily obtain analytical and numerical results for flavor observables in the BSM model of his choice.

Acknowledgments We thank Asmaa Abada, Martin Hirsch, Farvah Mahmoudi, Manuel E. Krauss, Kilian Nickel, Ben O'Leary and Cédric Weiland for helpful discussions. FS is supported by the BMBF PT DESY Verbundprojekt 05H2013-THEORIE 'Vergleich von LHCDaten mit supersymmetrischen Modellen'. WP is supported by the DFG, Project No. PO-1337/3-1. AV is partially supported by the EXPL/FIS-NUC/0460/2013 project financed by the Portuguese FCT.

Open Access This article is distributed under the terms of the Creative Commons Attribution License which permits any use, distribution, and reproduction in any medium, provided the original author(s) and the source are credited.

Funded by $\mathrm{SCOAP}^{3}$ / License Version CC BY 4.0.

\section{Appendix A: Lagrangian}

In this section we present our notation and conventions for the operators (and their corresponding Wilson coefficients) implemented in PreSARAH. Although a more complete list of flavor violating operators can be built, we will concentrate on those implemented in PreSARAH. If necessary, the user can extend it by adding his/her own operators.

The interaction Lagrangian relevant for flavor violating processes can be written as

$\mathcal{L}_{\mathrm{FV}}=\mathcal{L}_{\mathrm{LFV}}+\mathcal{L}_{\mathrm{QFV}}$

The first piece contains the operators that can trigger lepton flavor violation whereas the second piece contains the operators responsible for quark flavor violation.

The general Lagrangian relevant for lepton flavor violation can be written as

$\mathcal{L}_{\mathrm{LFV}}=\mathcal{L}_{\ell \ell \gamma}+\mathcal{L}_{\ell \ell Z}+\mathcal{L}_{\ell \ell h}+\mathcal{L}_{4 \ell}+\mathcal{L}_{2 \ell 2 q}$.

The first term contains the $\ell-\ell-\gamma$ interaction, given by

$$
\begin{aligned}
\mathcal{L}_{\ell \ell \gamma}= & e \bar{\ell}_{\beta}\left[\gamma^{\mu}\left(K_{1}^{L} P_{L}+K_{1}^{R} P_{R}\right)\right. \\
& \left.+i m_{\ell_{\alpha}} \sigma^{\mu \nu} q_{v}\left(K_{2}^{L} P_{L}+K_{2}^{R} P_{R}\right)\right] \ell_{\alpha} A_{\mu}+\text { h.c. }
\end{aligned}
$$

Here $e$ is the electric charge, $q$ the photon momentum, $P_{L, R}=\frac{1}{2}\left(1 \mp \gamma_{5}\right)$ are the usual chirality projectors and $\ell_{\alpha, \beta}$ denote the lepton flavors. For practical reasons, we will always consider the photonic contributions independently, and we will not include them in other vector operators. On the contrary, the $Z$ - and Higgs boson contributions will be included whenever possible. Therefore, the $\ell-\ell-Z$ and $\ell-\ell-h$ interaction Lagrangians will only be used for observables involving real $Z$ - and Higgs bosons. These two Lagrangians can be written as

$$
\begin{aligned}
\mathcal{L}_{\ell \ell Z}= & \bar{\ell}_{\beta}\left[\gamma^{\mu}\left(R_{1}^{L} P_{L}+R_{1}^{R} P_{R}\right)\right. \\
& \left.+p^{\mu}\left(R_{2}^{L} P_{L}+R_{2}^{R} P_{R}\right)\right] \ell_{\alpha} Z_{\mu},
\end{aligned}
$$

where $p$ is the $\ell_{\beta}$ 4-momentum, and

$\mathcal{L}_{\ell \ell h}=\bar{\ell}_{\beta}\left(S_{L} P_{L}+S_{R} P_{R}\right) \ell_{\alpha} h$.

The general $4 \ell$ 4-fermion interaction Lagrangian can be written as

$$
\mathcal{L}_{4 \ell}=\sum_{\substack{I=S, V, T \\ X, Y=L, R}} A_{X Y}^{I} \bar{\ell}_{\beta} \Gamma_{I} P_{X} \ell_{\alpha} \bar{\ell}_{\delta} \Gamma_{I} P_{Y} \ell_{\gamma}+\text { h.c. }
$$

where $\ell_{\alpha, \beta, \gamma, \delta}$ denote the lepton flavors and $\Gamma_{S}=1, \Gamma_{V}=$ $\gamma_{\mu}$ and $\Gamma_{T}=\sigma_{\mu \nu}$. We omit flavor indices in the Wilson coefficients for the sake of clarity. This Lagrangian contains the most general form compatible with Lorentz invariance. The Wilson coefficients $A_{L R}^{S}$ and $A_{R L}^{S}$ were included in [57], but absent in $[42,58]$. As previously stated, the coefficients in Eq. (A.6) do not include photonic contributions, but they include Z-boson and scalar ones. Finally, the general $2 \ell 2 q$ four fermion interaction Lagrangian at the quark level is given by

$\mathcal{L}_{2 \ell 2 q}=\mathcal{L}_{2 \ell 2 d}+\mathcal{L}_{2 \ell 2 u}$

where

$$
\begin{aligned}
\mathcal{L}_{2 \ell 2 d}= & \sum_{\substack{I=S, V, T \\
X, Y=L, R}} B_{X Y}^{I} \bar{\ell}_{\beta} \Gamma_{I} P_{X} \ell_{\alpha} \bar{d}_{\gamma} \Gamma_{I} P_{Y} d_{\gamma}+\text { h.c. } \\
\mathcal{L}_{2 \ell 2 u}= & \left.\mathcal{L}_{2 \ell 2 d}\right|_{d \rightarrow u, B \rightarrow C} .
\end{aligned}
$$

Here $d_{\gamma}$ denotes the d-quark flavor.

Let us now consider the Lagrangian relevant for quark flavor violation. This can be written as

$$
\begin{aligned}
\mathcal{L}_{\mathrm{QFV}}= & \mathcal{L}_{q q \gamma}+\mathcal{L}_{q q g}+\mathcal{L}_{4 d}+\mathcal{L}_{2 d 2 l} \\
& +\mathcal{L}_{2 d 2 v}+\mathcal{L}_{d u \ell v}+\mathcal{L}_{d d H} .
\end{aligned}
$$

The first two terms correspond to operators that couple quark bilinears to massless gauge bosons. These are 
$\mathcal{L}_{q q \gamma}=e\left[\bar{d}_{\beta} \sigma_{\mu \nu}\left(m_{d_{\beta}} Q_{1}^{L} P_{L}+m_{d_{\alpha}} Q_{1}^{R} P_{R}\right) d_{\alpha}\right] F^{\mu \nu}$

$\mathcal{L}_{q q g}=g_{s}\left[\bar{d}_{\beta} \sigma_{\mu \nu}\left(m_{d_{\beta}} Q_{2}^{L} P_{L}+m_{d_{\alpha}} Q_{2}^{R} P_{R}\right) T^{a} d_{\alpha}\right] G_{a}^{\mu \nu}$.

Here $T^{a}$ are $S U$ (3) matrices. The Wilson coefficients $Q_{1,2}^{L, R}$ can be easily related to the usual $C_{7.8}^{(\prime)}$ coefficients, sometimes normalized with an additional $\frac{1}{16 \pi^{2}}$ factor. The $4 d$ four fermion interaction Lagrangian can be written as

$$
\mathcal{L}_{4 d}=\sum_{\substack{I=S, V, T \\ X, Y=L, R}} D_{X Y}^{I} \bar{d}_{\beta} \Gamma_{I} P_{X} d_{\alpha} \bar{d}_{\delta} \Gamma_{I} P_{Y} d_{\gamma}+\text { h.c. }
$$

where $d_{\alpha, \beta, \gamma, \delta}$ denote the lepton flavors. Again, we omit flavor indices in the Wilson coefficients for the sake of clarity. The $2 d 2 \ell$ four fermion interaction Lagrangian is given by

$$
\mathcal{L}_{2 d 2 \ell}=\sum_{\substack{I=S, V, T \\ X, Y=L, R}} E_{X Y}^{I} \bar{d}_{\beta} \Gamma_{I} P_{X} d_{\alpha} \bar{\ell}_{\gamma} \Gamma_{I} P_{Y} \ell_{\gamma}+\text { h.c.. }
$$

Here $\ell_{\gamma}$ denotes the lepton flavor. $\mathcal{L}_{2 d 2 \ell}$ should not be confused with $\mathcal{L}_{2 \ell 2 d}$. In the former case one has QFV operators, whereas in the latter one has LFV operators. This distinction has been made for practical reasons. The $2 d 2 v$ and $d u \ell v$ terms of the QFV Lagrangian are

$$
\begin{aligned}
& \mathcal{L}_{2 d 2 v}=\sum_{X, Y=L, R} F_{X Y}^{V} \bar{d}_{\beta} \gamma_{\mu} P_{X} d_{\alpha} \bar{v}_{\gamma} \gamma^{\mu} P_{Y} v_{\gamma}+\text { h.c. } \\
& \mathcal{L}_{d u \ell v}=\sum_{\substack{I=S, V \\
X, Y=L, R}} G_{X Y}^{I} \bar{d}_{\beta} \Gamma_{I} P_{X} u_{\alpha} \bar{\ell}_{\gamma} \Gamma_{I} P_{Y} v_{\gamma}+\text { h.c.. } \\
&
\end{aligned}
$$

Note that we have not introduced scalar or tensor $2 d 2 v$ operators, nor tensor $d u \ell v$ ones, and that lepton flavor (denoted by the index $\gamma$ ) is conserved in these operators. Finally, we have also included a term in the Lagrangian accounting for operators of the type $(\bar{d} \Gamma d) S$ and $(\bar{d} \Gamma d) P$, where $S(P)$ is a virtual ${ }^{10}$ scalar (pseudoscalar) state. This piece can be written as

$$
\begin{aligned}
\mathcal{L}_{d d H}= & \bar{d}_{\beta}\left(H_{L}^{S} P_{L}+H_{R}^{S} P_{R}\right) d_{\alpha} S \\
& +\bar{d}_{\beta}\left(H_{L}^{P} P_{L}+H_{R}^{P} P_{R}\right) d_{\alpha} P .
\end{aligned}
$$

\footnotetext{
${ }^{10}$ We would like to emphasize that our implementation of these operators is only valid for virtual scalars and pseudoscalars. They have been introduced in order to provide the 1-loop vertices necessary for the computation of the double penguin contributions to $\Delta M_{B_{q}}$. Therefore, they are not valid for observables in which the scalar or pseudoscalar states are real particles.
}

\section{Appendix B: Operators available by default in the SPheno output of SARAH}

The operators presented in Appendix A have been implemented by using the results of PreSARAH in SARAH. Those are exported to SPheno. We give in the following the list of all internal names for these operators, which can be used in the calculation of new flavor observables.

\section{B.1 2-Fermion-1-Boson operators}

These operators are arrays with either two or three elements. While operators involving vector bosons have always dimension $3 \times 3$, those with scalars have dimension $3 \times 3 \times n_{g} . n_{g}$ is the number of generations of the considered scalar and for $n_{g}=1$ the last index is dropped.

\begin{tabular}{lll}
\hline$\left(\bar{d}_{\beta} \sigma_{\mu \nu} \Gamma d_{\alpha}\right) F^{\mu \nu}$ and $\left(\bar{d}_{\beta} \sigma_{\mu \nu} \Gamma d_{\alpha}\right) G^{\mu \nu}$ & Name \\
\hline Variable & Operator & $Q_{1}^{L}$ \\
\hline CC7 & $e m_{d_{\beta}}\left(\bar{d}_{\beta} \sigma_{\mu \nu} P_{L} d_{\alpha}\right) F^{\mu \nu}$ & $Q_{1}^{R}$ \\
CC7p & $e m_{d_{\alpha}}\left(\bar{d}_{\beta} \sigma_{\mu \nu} P_{R} d_{\alpha}\right) F^{\mu \nu}$ & $Q_{2}^{L}$ \\
CC8 & $g_{s} m_{d_{\beta}}\left(\bar{d}_{\beta} \sigma_{\mu \nu} P_{L} d_{\alpha}\right) G^{\mu \nu}$ & $Q_{2}^{R}$ \\
CC8p & $g_{s} m_{d_{\alpha}}\left(\bar{d}_{\beta} \sigma_{\mu \nu} P_{R} d_{\alpha}\right) G^{\mu \nu}$ & \\
\hline
\end{tabular}

These operators are derived by PreSARAH with the following input files

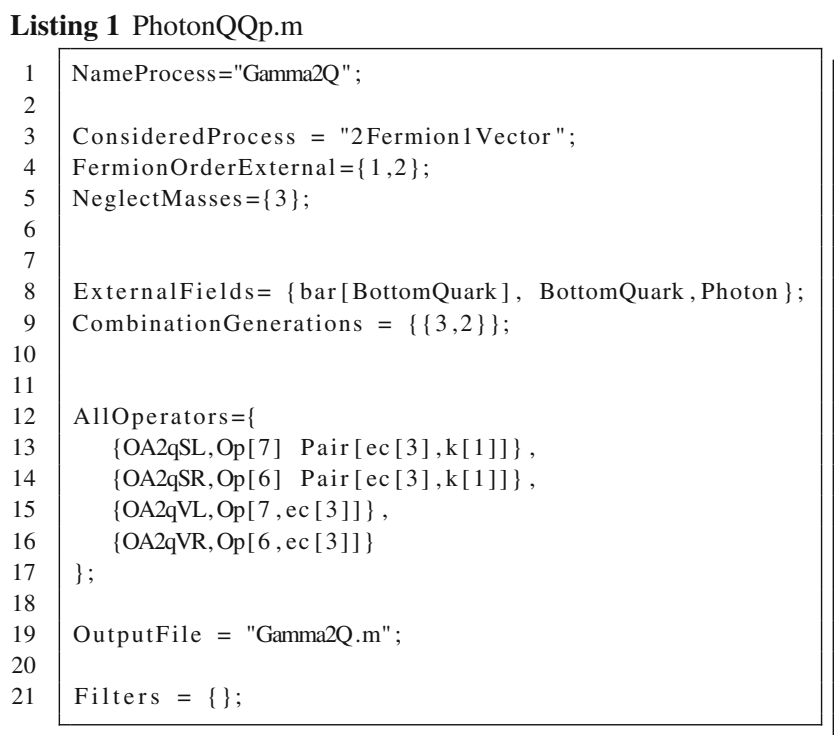

\section{Listing 2 GluonQQp.m}

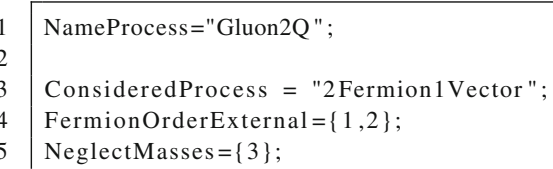




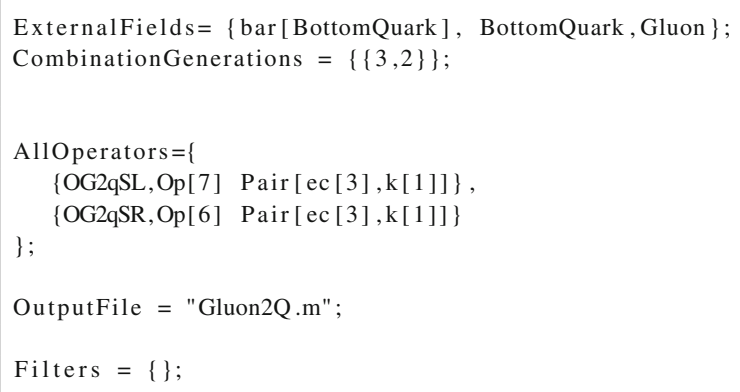

The normalization is changed to match the standard definitions by

\section{Listing 3 Photon_wrapper_QFV.m}

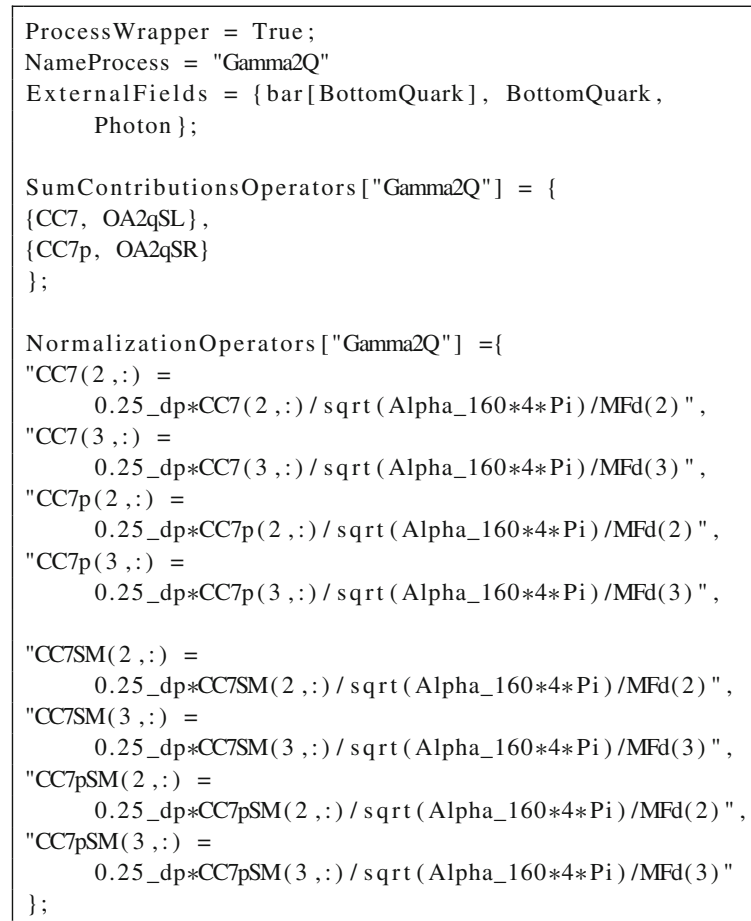

\section{Listing 4 Gluon_wrapper.m}

\begin{tabular}{|c|}
\hline ProcessWrapper = True \\
\hline NameProcess = "Gluon2Q" \\
\hline $\begin{array}{l}\text { ExternalFields }=\{\text { bar [BottomQuark }] \text {, BottomQuark, } \\
\text { Gluon }\}\end{array}$ \\
\hline \\
\hline SumContributionsOperators ["Gluon2Q"] $=\{$ \\
\hline$\{\mathrm{CC} 8, \mathrm{OG} 2 \mathrm{qSL}\}$ \\
\hline$\{\mathrm{CC} 8 \mathrm{p}$, OG2qSR $\}\}$ \\
\hline \\
\hline NormalizationOperators ["Gluon2Q"] $=\{$ \\
\hline "CC8 $(2,:)=$ \\
\hline $0.25 \_\mathrm{dp} * \mathrm{CC} 8(2,:) / \mathrm{sqrt}\left(\mathrm{AlphaS} \_160 * 4 * \mathrm{Pi}\right) / \operatorname{MFd}(2) "$, \\
\hline "CC8 $(3,:)=$ \\
\hline $0.25 \_\mathrm{dp} * \mathrm{CC} 8(3,:) / \mathrm{sqrt}\left(\mathrm{AlphaS} \_160 * 4 * \mathrm{Pi}\right) / \mathrm{MFd}(3)$ ", \\
\hline "CC8p $(2,:)=$ \\
\hline $0.25 \_\mathrm{dp} * \mathrm{CC} 8 \mathrm{p}(2,:) / \mathrm{sqrt}\left(\mathrm{AlphaS} \_160 * 4 * \mathrm{Pi}\right) / \mathrm{MFd}(2) "$, \\
\hline $\operatorname{cC} 8 \mathrm{p}(3,:)=$ \\
\hline $0.25 \_\mathrm{dp} * \mathrm{CC} 8 \mathrm{p}(3,:) / \mathrm{sqrt}\left(\mathrm{AlphaS} \_160 * 4 * \mathrm{Pi}\right) / \mathrm{MFd}(3)$ ", \\
\hline
\end{tabular}

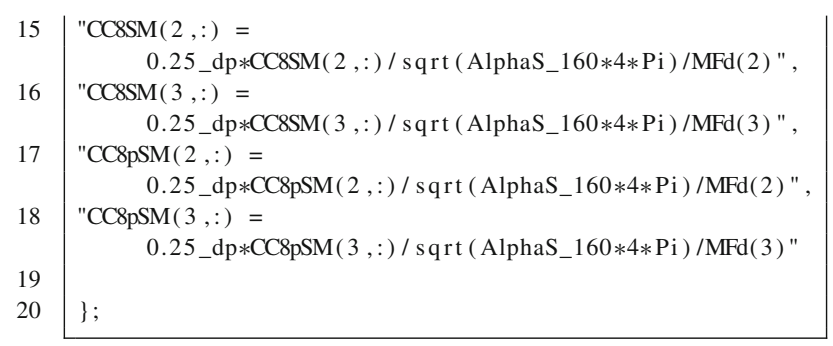

\begin{tabular}{lll}
\hline $\bar{\ell}_{\beta}\left(q^{2} \gamma^{\mu}+i m_{\ell_{\alpha}} \sigma^{\mu v} q_{\nu}\right) \ell_{\alpha} A_{\mu}$ \\
\hline Variable & Operator & Name \\
\hline K2L & $e m_{\ell_{\alpha}}\left(\bar{\ell}_{\beta} \sigma_{\mu \nu} P_{L} \ell_{\alpha}\right) q^{v} A^{\mu}$ & $K_{2}^{L}$ \\
K2R & $e m_{\ell_{\alpha}}\left(\bar{\ell}_{\beta} \sigma_{\mu \nu} P_{R} \ell_{\alpha}\right) q^{v} A^{\mu}$ & $K_{2}^{L}$ \\
K1L & $q^{2}\left(\bar{\ell}_{\beta} \gamma_{\mu} P_{L} \ell_{\alpha}\right) A^{\mu}$ & $K_{1}^{L}$ \\
K1R & $q^{2}\left(\bar{\ell}_{\beta} \gamma_{\nu} P_{R} \ell_{\alpha}\right) A^{\mu}$ & $K_{1}^{R}$ \\
\hline
\end{tabular}

These operators are derived by PreSARAH with the following input files

Listing 5 PhotonLLp.m

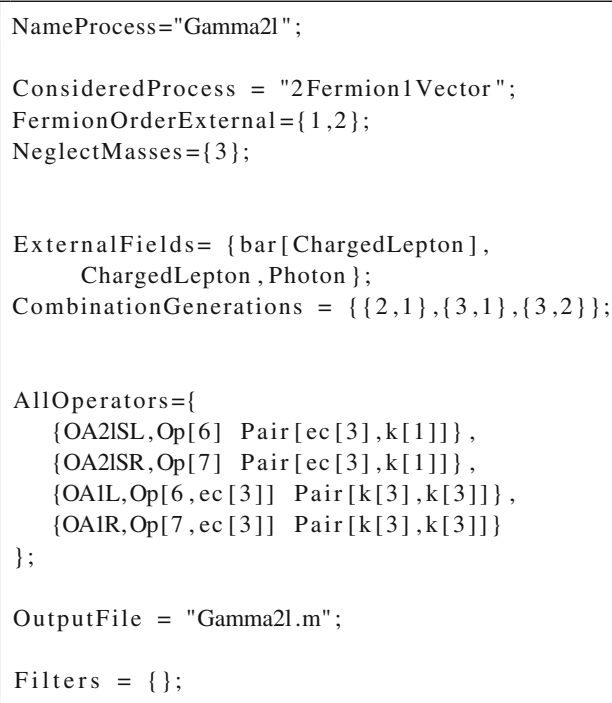

The normalization is changed to match the standard definitions by

\section{Listing 6 Photon_wrapper_LFV.m}

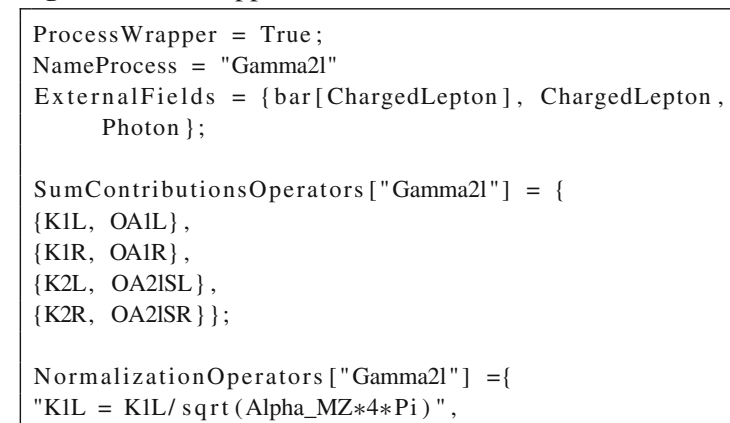




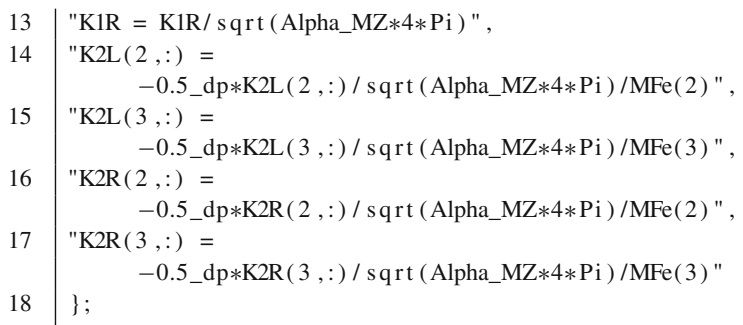

\section{$(\bar{\ell} \Gamma \ell) Z$}

\begin{tabular}{llc}
\hline Variable & Operator & Name \\
\hline OZ21VL & $\left(\bar{\ell} \gamma^{\mu} P_{L} \ell\right) Z_{\mu}$ & $R_{1}^{L}$ \\
OZ21VR & $\left(\bar{\ell} \gamma^{\mu} P_{R} \ell\right) Z_{\mu}$ & $R_{1}^{R}$ \\
OZ21SL & $\left(\bar{\ell} p^{\mu} P_{L} \ell\right) Z_{\mu}$ & $R_{2}^{L}$ \\
OZ21SR & $\left(\bar{\ell} p^{\mu} P_{R} \ell\right) Z_{\mu}$ & $R_{2}^{R}$ \\
\hline
\end{tabular}

In the following we omit flavor indices for the sake of simplicity. These operators are derived by PreSARAH with the following input files

\section{Listing 7 Z21.m}

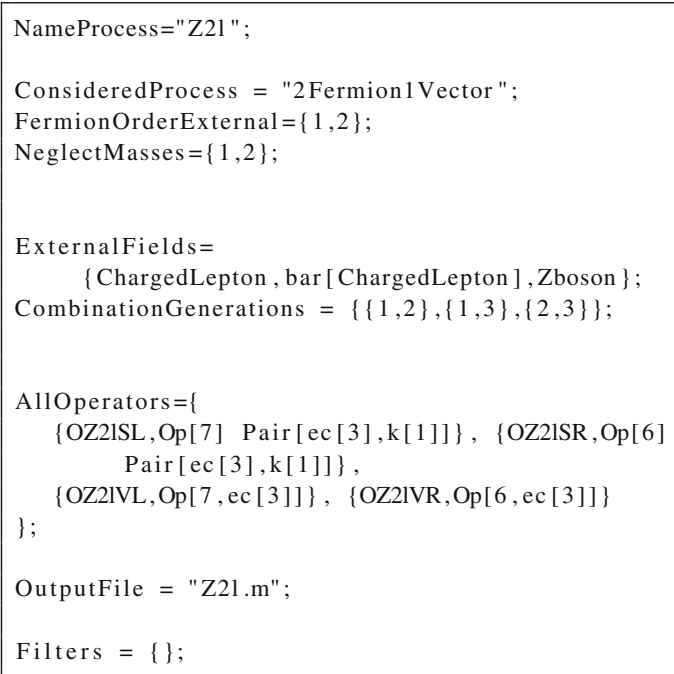

\begin{tabular}{lll}
\hline$(\bar{\ell} \Gamma \ell) h$ & & \\
\hline Variable & Operator & Name \\
\hline OH21SL & $\bar{\ell} P_{L} \ell h$ & $S_{L}$ \\
OH21SR & $\bar{\ell} P_{R} \ell h$ & $S_{R}$ \\
\hline
\end{tabular}

These operators are derived by PreSARAH with the following input files

\section{Listing 8 H2l.m}

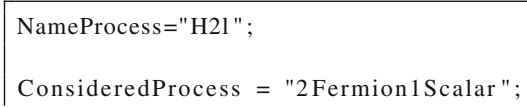

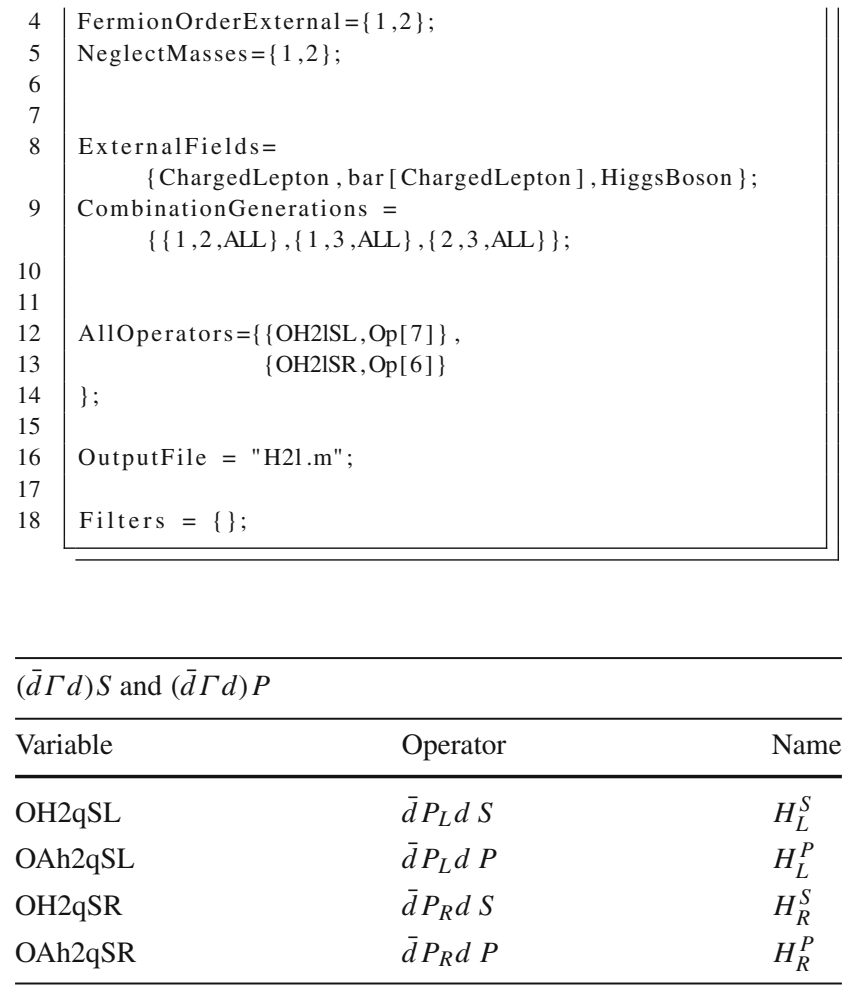

\begin{tabular}{llc}
\hline Variable & Operator & Name \\
\hline OH2qSL & $\bar{d} P_{L} d S$ & $H_{L}^{S}$ \\
OAh2qSL & $\bar{d} P_{L} d P$ & $H_{L}^{P}$ \\
OH2qSR & $\bar{d} P_{R} d S$ & $H_{R}^{S}$ \\
OAh2qSR & $\bar{d} P_{R} d P$ & $H_{R}^{P}$ \\
\hline
\end{tabular}

These auxiliary ${ }^{11}$ operators are derived by PreSARAH with the following input files

\section{Listing 9 H2q.m}

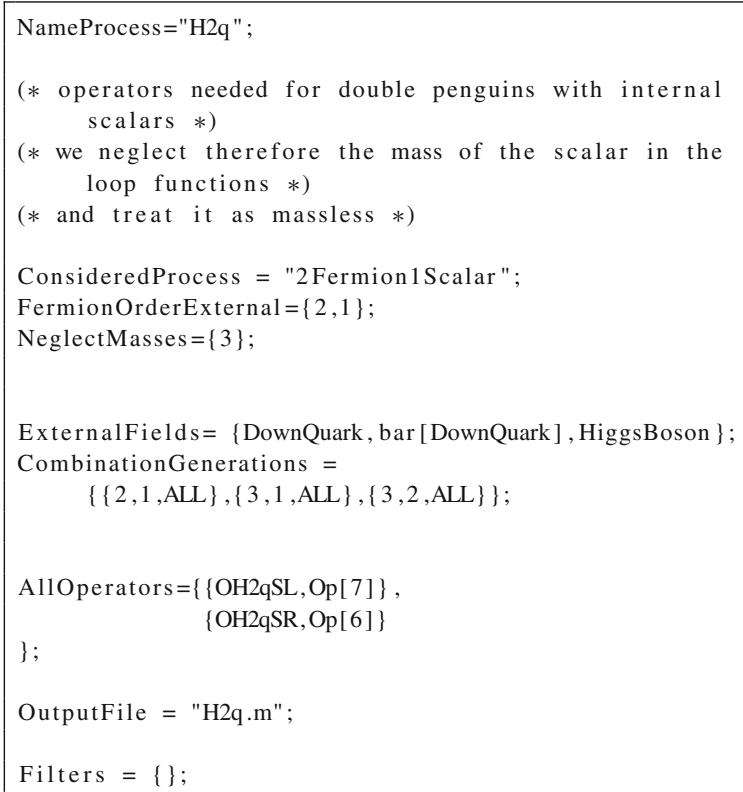

$\overline{11}$ The $(\bar{d} \Gamma d) S$ and $(\bar{d} \Gamma d) P$ operators have been introduced to compute double penguin corrections to $\Delta M_{B_{q}}$, where $S$ and $P$ appear as intermediate (virtual) particles. They should not be used in processes where the scalar or pseudoscalar states are real particles because the loop functions are calculated with vanishing external momenta. 


\section{Listing 10 A2q.m}

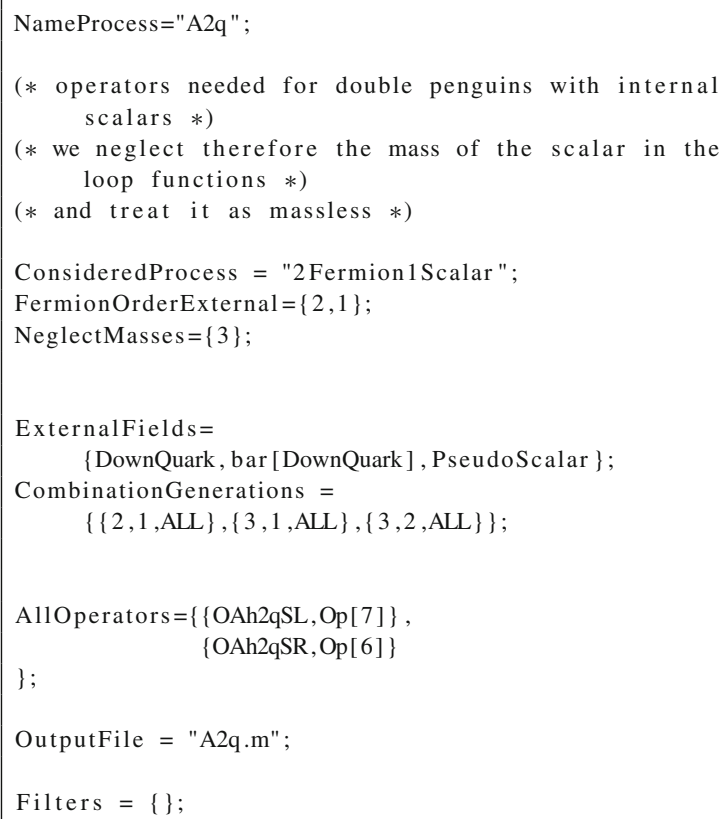

\section{B.2 4-Fermion operators}

All operators listed below carry four indices and have dimension $3 \times 3 \times 3 \times 3$. In addition, the user can access the different contributions of all operators from tree-level diagrams, as well as penguin and box diagrams. The name conventions are as follows: for each operator op the additional parameter exist

- TSop: tree-level contributions with scalar propagator

- TVop: tree-level contributions with scalar propagator

- PSop: sum of penguin and self-energy contributions with scalar propagator

- PVop: sum of penguin and self-energy contributions with scalar propagator

- Bop: box contributions.

We will denote the 4-fermion operators involving two leptons and two down-type quarks depending on whether they lead to LFV or to QFV processes: $\ell \ell d d$ for LFV and $d d \ell \ell$ for QFThese operators are derived by PreSARAH with the following input files

\section{Listing 11 2d2L.m}

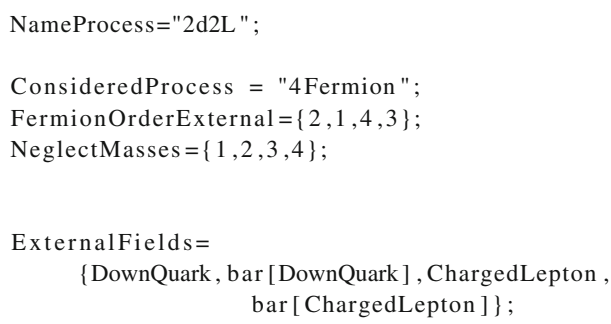

$\overline{(\bar{d} \Gamma d)\left(\bar{\ell} \Gamma^{\prime} \ell\right) \text { and }(\bar{d} \Gamma d)\left(\bar{v} \Gamma^{\prime} v\right)}$

\begin{tabular}{|c|c|c|}
\hline Variable & Operator & Name \\
\hline OddllSLL & $\left(\bar{d} P_{L} d\right)\left(\bar{\ell} P_{L} \ell\right)$ & $E_{L L}^{S}$ \\
\hline OddllSRR & $\left(\bar{d} P_{R} d\right)\left(\bar{\ell} P_{R} \ell\right)$ & $E_{R R}^{S}$ \\
\hline OddllSLR & $\left(\bar{d} P_{L} d\right)\left(\bar{\ell} P_{R} \ell\right)$ & $E_{L R}^{S}$ \\
\hline OddllSRL & $\left(\bar{d} P_{R} d\right)\left(\bar{\ell} P_{L} \ell\right)$ & $E_{R L}^{S}$ \\
\hline OddllVLL & $\left(\bar{d} \gamma_{\mu} P_{L} d\right)\left(\bar{\ell} \gamma^{\mu} P_{L} \ell\right)$ & $E_{L L}^{V}$ \\
\hline OddllVRR & $\left(\bar{d} \gamma_{\mu} P_{R} d\right)\left(\bar{\ell} \gamma^{\mu} P_{R} \ell\right)$ & $E_{R R}^{V}$ \\
\hline OddllVLR & $\left(\bar{d} \gamma_{\mu} P_{L} d\right)\left(\bar{\ell} \gamma^{\mu} P_{R} \ell\right)$ & $E_{L R}^{V}$ \\
\hline OddllVRL & $\left(\bar{d} \gamma_{\mu} P_{R} d\right)\left(\bar{\ell} \gamma^{\mu} P_{L} \ell\right)$ & $E_{R L}^{V}$ \\
\hline OddllTLL & $\left(\bar{d} \sigma_{\mu \nu} P_{L} d\right)\left(\bar{\ell} \sigma^{\mu \nu} P_{L} \ell\right)$ & $E_{L L}^{T}$ \\
\hline OddllTRR & $\left(\bar{d} \sigma_{\mu \nu} P_{R} d\right)\left(\bar{\ell} \sigma^{\mu \nu} P_{R} \ell\right)$ & $E_{R R}^{T}$ \\
\hline OddllTLR & $\left(\bar{d} \sigma_{\mu \nu} P_{L} d\right)\left(\bar{\ell} \sigma^{\mu \nu} P_{R} \ell\right)$ & $E_{L R}^{T}$ \\
\hline OddllTRL & $\left(\bar{d} \sigma_{\mu \nu} P_{R} d\right)\left(\bar{\ell} \sigma^{\mu \nu} P_{L} \ell\right)$ & $E_{R L}^{T}$ \\
\hline OddvvVLL & $\left(\bar{d} \gamma_{\mu} P_{L} d\right)\left(\bar{v} \gamma^{\mu} P_{R} v\right)$ & $F_{L L}^{V}$ \\
\hline OddvvVRR & $\left(\bar{d} \gamma_{\mu} P_{R} d\right)\left(\bar{\nu} \gamma^{\mu} P_{R} v\right)$ & $F_{R R}^{V}$ \\
\hline OddvvVLR & $\left(\bar{d} \gamma_{\mu} P_{L} d\right)\left(\bar{v} \gamma^{\mu} P_{R} v\right)$ & $F_{L R}^{V}$ \\
\hline OddvvVRL & $\left(\bar{d} \gamma_{\mu} P_{R} d\right)\left(\bar{v} \gamma^{\mu} P_{L} v\right)$ & $F_{R L}^{V}$ \\
\hline
\end{tabular}

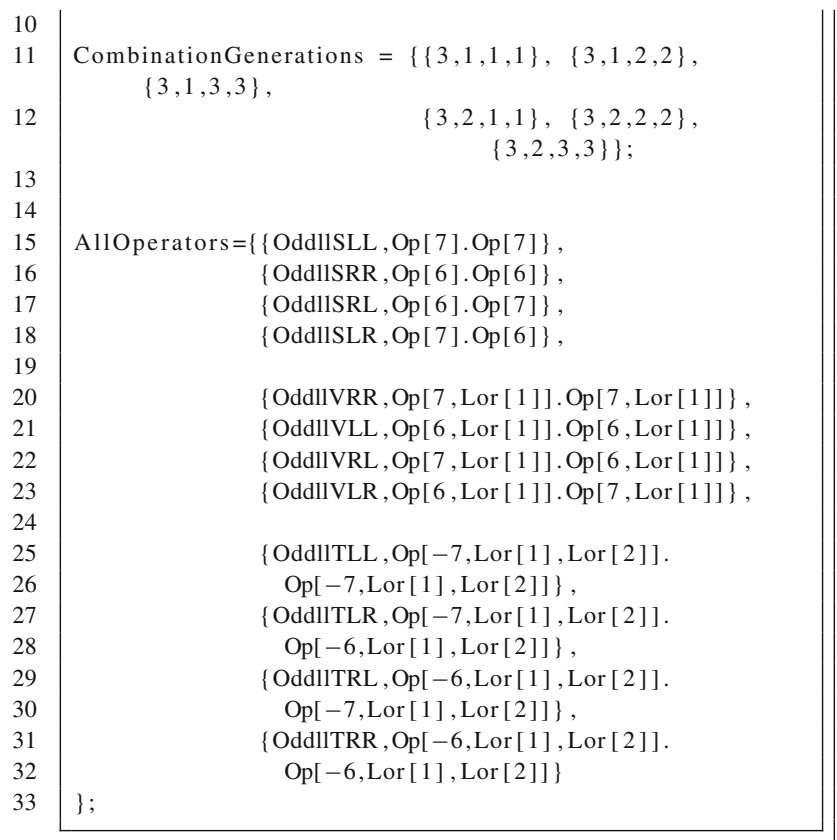

\section{Listing 12 2d2nu.m}

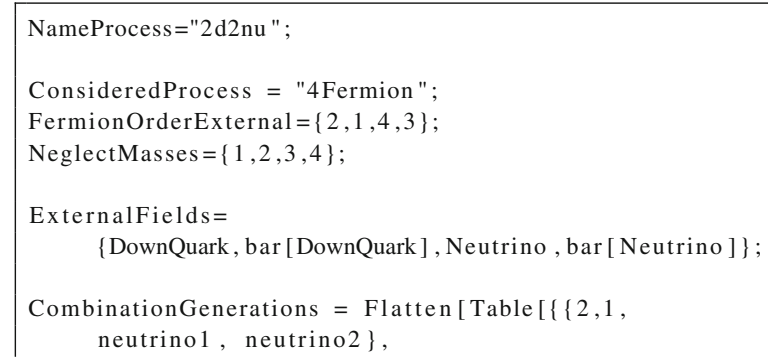




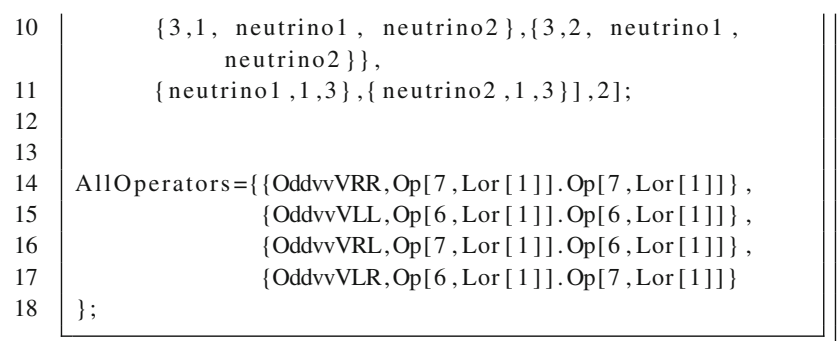

$(\bar{\ell} \Gamma \ell)\left(\bar{d} \Gamma^{\prime} d\right)$ and $(\bar{\ell} \Gamma \ell)\left(\bar{u} \Gamma^{\prime} u\right)$

\begin{tabular}{lll}
\hline Variable & Operator & Name \\
\hline OllddSLL & $\left(\bar{\ell} P_{L} \ell\right)\left(\bar{d} P_{L} d\right)$ & $B_{L L}^{S}$ \\
OllddSRR & $\left(\bar{\ell} P_{R} \ell\right)\left(\bar{d} P_{R} d\right)$ & $B_{R R}^{S}$ \\
OllddSRL & $\left(\bar{\ell} P_{R} \ell\right)\left(\bar{d} P_{L} d\right)$ & $B_{R L}^{S}$ \\
OllddSLR & $\left(\bar{\ell} P_{L} \ell\right)\left(\bar{d} P_{R} d\right)$ & $B_{L R}^{S}$ \\
OllddVLL & $\left(\bar{\ell} \gamma_{\mu} P_{L} \ell\right)\left(\bar{d} \gamma^{\mu} P_{L} d\right)$ & $B_{L L}^{V}$ \\
OlluuVLL & $\left(\bar{\ell} \gamma_{\mu} P_{L} \ell\right)\left(\bar{u} \gamma^{\mu} P_{L} u\right)$ & $C_{L L}^{V}$ \\
OllddVRR & $\left(\bar{\ell} \gamma_{\mu} P_{R} \ell\right)\left(\bar{d} \gamma^{\mu} P_{R} d\right)$ & $B_{R R}^{V}$ \\
OllddVLR & $\left(\bar{\ell} \gamma_{\mu} P_{L} \ell\right)\left(\bar{d} \gamma^{\mu} P_{R} d\right)$ & $B_{L R}^{V}$ \\
OlluuVLR & $\left(\bar{\ell} \gamma_{\mu} P_{L} \ell\right)\left(\bar{u} \gamma^{\mu} P_{R} u\right)$ & $C_{L R}^{V}$ \\
OllddVRL & $\left(\bar{\ell} \gamma_{\mu} P_{R} \ell\right)\left(\bar{d} \gamma^{\mu} P_{L} d\right)$ & $B_{R L}^{V}$ \\
OllddTLL & $\left(\bar{\ell} \sigma_{\mu \nu} P_{L} \ell\right)\left(\bar{d} \sigma^{\mu \nu} P_{L} d\right)$ & $B_{L L}^{T}$ \\
OllddTRR & $\left(\bar{\ell} \sigma_{\mu \nu} P_{R} \ell\right)\left(\bar{d} \sigma^{\mu \nu} P_{R} d\right)$ & $B_{R R}^{T}$ \\
OllddTLR & $\left(\bar{\ell} \sigma_{\mu \nu} P_{L} \ell\right)\left(\bar{d} \sigma^{\mu \nu} P_{R} d\right)$ & $B_{L R}^{T}$ \\
OllddTRL & $\left(\bar{\ell} \sigma_{\mu \nu} P_{R} \ell\right)\left(\bar{d} \sigma^{\mu v} P_{L} d\right)$ & $B_{R L}^{T}$ \\
OlluuSLL & $\left(\bar{\ell} P_{L} \ell\right)\left(\bar{u} P_{L} u\right)$ & $C_{L L}^{S}$ \\
OlluuSRR & $\left(\bar{\ell} P_{R} \ell\right)\left(\bar{u} P_{R} u\right)$ & $C_{R R}^{S}$ \\
OlluuSRL & $\left(\bar{\ell} P_{R} \ell\right)\left(\bar{u} P_{L} u\right)$ & $C_{R L}^{S}$ \\
OlluuSLR & $\left(\bar{\ell} P_{L} \ell\right)\left(\bar{u} P_{R} u\right)$ & $C_{L R}^{S}$ \\
OlluuVLL & $\left(\bar{\ell} \gamma_{\mu} P_{L} \ell\right)\left(\bar{u} \gamma^{\mu} P_{L} u\right)$ & $C_{L L}^{V}$ \\
OlluuVRR & $\left(\bar{\ell} \gamma_{\mu} P_{R} \ell\right)\left(\bar{u} \gamma^{\mu} P_{R} u\right)$ & $C_{R R}^{V}$ \\
OlluuVLR & $\left(\bar{\ell} \gamma_{\mu} P_{L} \ell\right)\left(\bar{u} \gamma^{\mu} P_{R} u\right)$ & $C_{L R}^{V}$ \\
OlluuVRL & $\left(\bar{\ell} \gamma_{\mu} P_{R} \ell\right)\left(\bar{u} \gamma^{\mu} P_{L} u\right)$ & $C_{R L}^{V}$ \\
OlluuTLL & $\left(\bar{\ell} \sigma_{\mu \nu} P_{L} \ell\right)\left(\bar{u} \sigma^{\mu \nu} P_{L} u\right)$ & $C_{L L}^{T}$ \\
OlluuTRR & $\left(\bar{\ell} \sigma_{\mu \nu} P_{R} \ell\right)\left(\bar{u} \sigma^{\mu \nu} P_{R} u\right)$ & $C_{R R}^{T}$ \\
OlluuTLR & $\left(\bar{\ell} \sigma_{\mu \nu} P_{L} \ell\right)\left(\bar{u} \sigma^{\mu v} P_{R} u\right)$ & $C_{L R}^{T}$ \\
OlluuTRL & $\left(\bar{\ell} \sigma_{\mu \nu} P_{R} \ell\right)\left(\bar{u} \sigma^{\mu \nu} P_{L} u\right)$ & $C_{R L}^{T}$ \\
\hline & &
\end{tabular}

Listing 13 2L2d.m

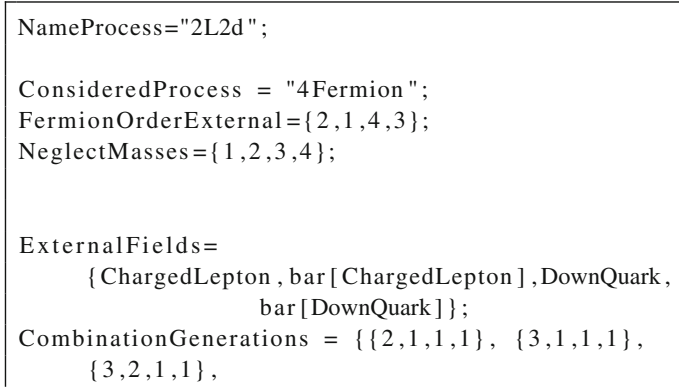

$\{2,1,2,2\},\{3,1,2,2\}$, $\{3,2,2,2\}\}$;

AllOperators $=\{\{$ OllddSLL, Op [7] $]$. Op [7] $\}$, \{OllddSRR, Op [6].Op[6]\}, \{OllddSRL, Op [6].Op[7]\}, \{OllddSLR , Op [7].Op[6]\},

\{OllddVRR, Op [7, Lor [1]].Op[7, Lor [1]]\}, $\{$ OllddVLL, Op[6, Lor [1]]. Op[6, Lor [1]]\}, \{OllddVRL, Op[7, Lor [1]].Op[6, Lor [1]]\}, $\{$ OllddVLR , Op [6, Lor [1]]. Op[7, Lor [1]]\},

\{OllddTLL, Op[ $-7, \operatorname{Lor}[1]$, Lor [2]].

Op[-7,Lor [1], Lor [2]]\},

\{OllddTLR, Op[ $-7, \operatorname{Lor}[1]$, Lor [2]].

Op[-6,Lor [1], Lor [2]]\},

\{OllddTRL, Op[ $-6, \operatorname{Lor}[1]$, Lor [2]].

Op[-7, Lor [1], Lor [2]]\},

\{OllddTRR , Op[ $-6, \operatorname{Lor}[1]$, Lor [2] ] .

\}

Op[ $[-6, \operatorname{Lor}[1], \operatorname{Lor}[2]]\}$

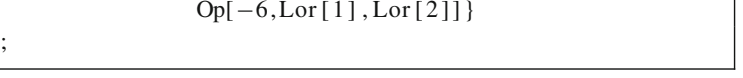

\section{Listing 14 2L2u.m}

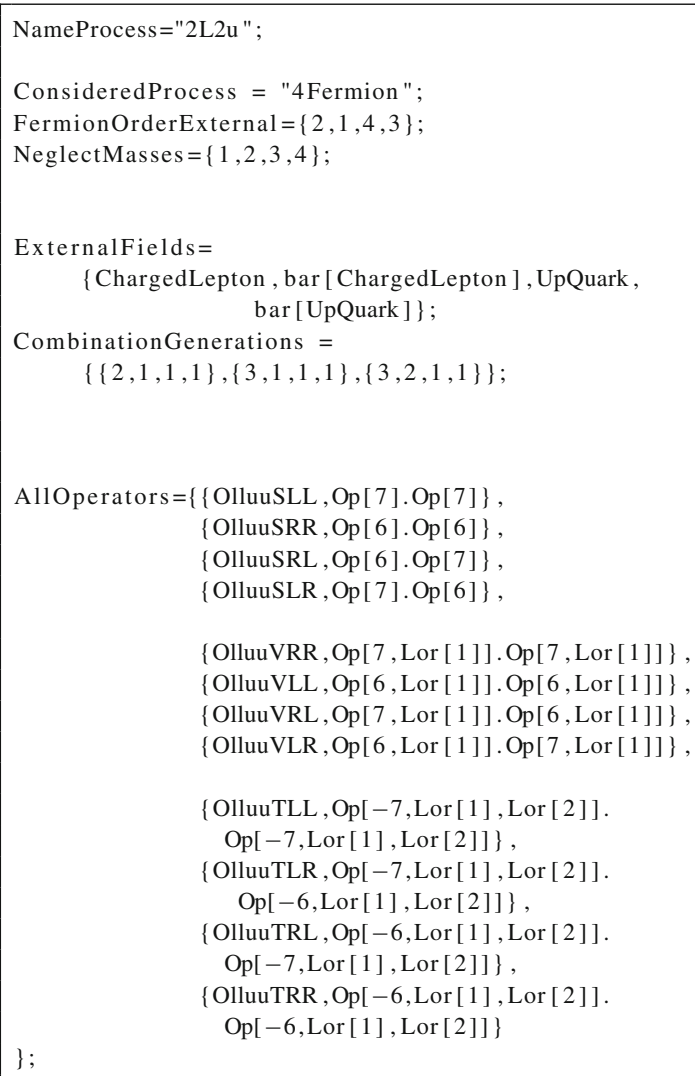

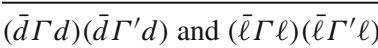

\begin{tabular}{lll}
\hline Variable & Operator & Name \\
\hline O4dSLL & $\left(\bar{d} P_{L} d\right)\left(\bar{d} P_{L} d\right)$ & $D_{L L}^{S}$ \\
O4dSRR & $\left(\bar{d} P_{R} d\right)\left(\bar{d} P_{R} d\right)$ & $D_{R R}^{S}$ \\
\hline
\end{tabular}




\begin{tabular}{lll}
\hline O4dSLR & $\left(\bar{d} P_{L} d\right)\left(\bar{d} P_{R} d\right)$ & $D_{L R}^{S}$ \\
O4dSRL & $\left(\bar{d} P_{R} d\right)\left(\bar{d} P_{L} d\right)$ & $D_{R L}^{S}$ \\
O4dVLL & $\left(\bar{d} \gamma_{\mu} P_{L} d\right)\left(\bar{d} \gamma^{\mu} P_{L} d\right)$ & $D_{L L}^{V}$ \\
O4dVRR & $\left(\bar{d} \gamma_{\mu} P_{R} d\right)\left(\bar{d} \gamma^{\mu} P_{R} d\right)$ & $D_{R R}^{V}$ \\
O4dVLR & $\left(\bar{d} \gamma_{\mu} P_{L} d\right)\left(\bar{d} \gamma^{\mu} P_{R} d\right)$ & $D_{L R}^{V}$ \\
O4dVRL & $\left(\bar{d} \gamma_{\mu} P_{R} d\right)\left(\bar{d} \gamma^{\mu} P_{L} d\right)$ & $D_{R L}^{V}$ \\
O4dTLL & $\left(\bar{d} \sigma_{\mu \nu} P_{L} d\right)\left(\bar{d} \sigma^{\mu \nu} P_{L} d\right)$ & $D_{L L}^{T}$ \\
O4dTRR & $\left(\bar{d} \sigma_{\mu \nu} P_{R} d\right)\left(\bar{d} \sigma^{\mu \nu} P_{R} d\right)$ & $D_{R R}^{T}$ \\
O4dTLR & $\left(\bar{d} \sigma_{\mu \nu} P_{L} d\right)\left(\bar{d} \sigma^{\mu \nu} P_{R} d\right)$ & $D_{L R}^{T}$ \\
O4dTRL & $\left(\bar{d} \sigma_{\mu \nu} P_{R} d\right)\left(\bar{d} \sigma^{\mu \nu} P_{L} d\right)$ & $D_{R L}^{T}$ \\
O4ISLL & $\left(\bar{\ell} P_{L} \ell\right)\left(\bar{\ell} P_{L} \ell\right)$ & $A_{L L}^{S}$ \\
O4ISRR & $\left(\bar{\ell} P_{R} \ell\right)\left(\bar{\ell} P_{R} \ell\right)$ & $A_{R R}^{S}$ \\
O4ISLR & $\left(\bar{\ell} P_{L} \ell\right)\left(\bar{\ell} P_{R} \ell\right)$ & $A_{L R}^{S}$ \\
O4ISRL & $\left(\bar{\ell} P_{R} \ell\right)\left(\bar{\ell} P_{L} \ell\right)$ & $A_{R L}^{S}$ \\
O4IVLL & $\left(\bar{\ell} \gamma_{\mu} P_{L} \ell\right)\left(\bar{\ell} \gamma^{\mu} P_{L} \ell\right)$ & $A_{L L}^{V}$ \\
O4IVRR & $\left(\bar{\ell} \gamma_{\mu} P_{R} \ell\right)\left(\bar{\ell} \gamma^{\mu} P_{R} \ell\right)$ & $A_{R R}^{V}$ \\
O4IVLR & $\left(\bar{\ell} \gamma_{\mu} P_{L} \ell\right)\left(\bar{\ell} \gamma^{\mu} P_{R} \ell\right)$ & $A_{L R}^{V}$ \\
O4IVRL & $\left(\bar{\ell} \gamma_{\mu} P_{R} \ell\right)\left(\bar{\ell} \gamma^{\mu} P_{L} \ell\right)$ & $A_{R L}^{V}$ \\
O4ITLL & $\left(\bar{\ell} \sigma_{\mu \nu} P_{L} \ell\right)\left(\bar{\ell} \sigma^{\mu \nu} P_{L} \ell\right)$ & $A_{L L}^{T}$ \\
O4ITRR & $\left(\bar{\ell} \sigma_{\mu \nu} P_{R} \ell\right)\left(\bar{\ell} \sigma^{\mu \nu} P_{R} \ell\right)$ & $A_{R R}^{T}$ \\
O4ITLR & $\left(\bar{\ell} \sigma_{\mu \nu} P_{L} \ell\right)\left(\bar{\ell} \sigma^{\mu \nu} P_{R} \ell\right)$ & $A_{L R}^{T}$ \\
O4ITRL & $\left(\bar{\ell} \sigma_{\mu \nu} P_{R} \ell\right)\left(\bar{\ell} \sigma^{\mu \nu} P_{L} \ell\right)$ & $A_{R L}^{T}$ \\
\hline & &
\end{tabular}

\section{Listing 15 4d.m}

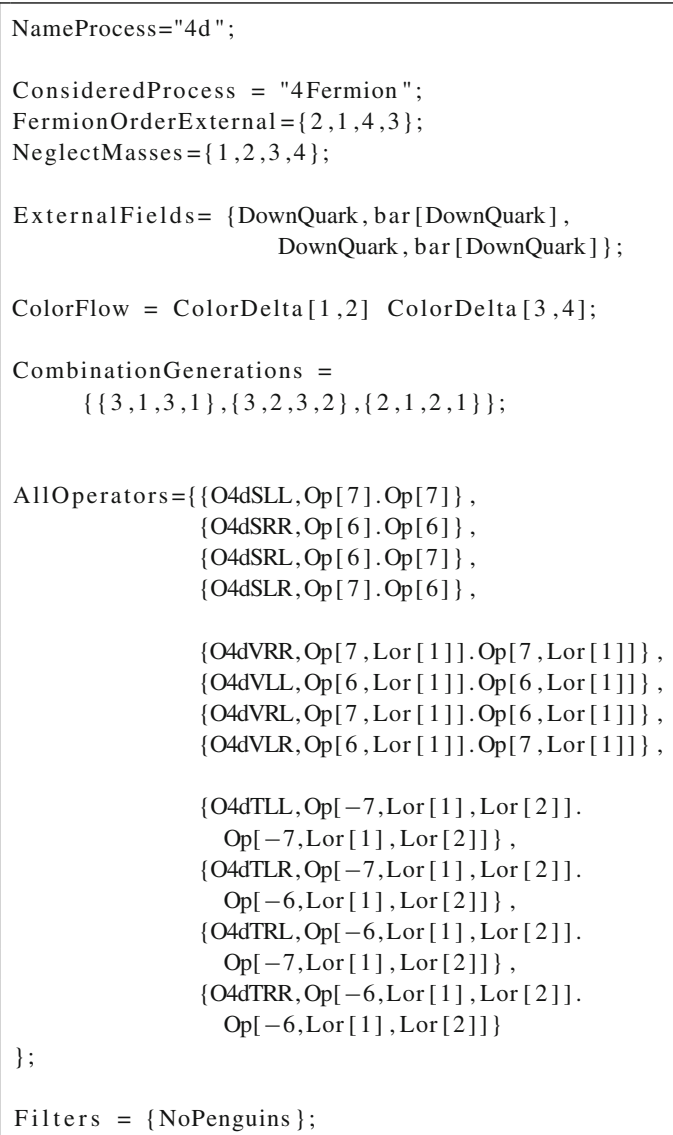

\section{Listing 16 4L.m}

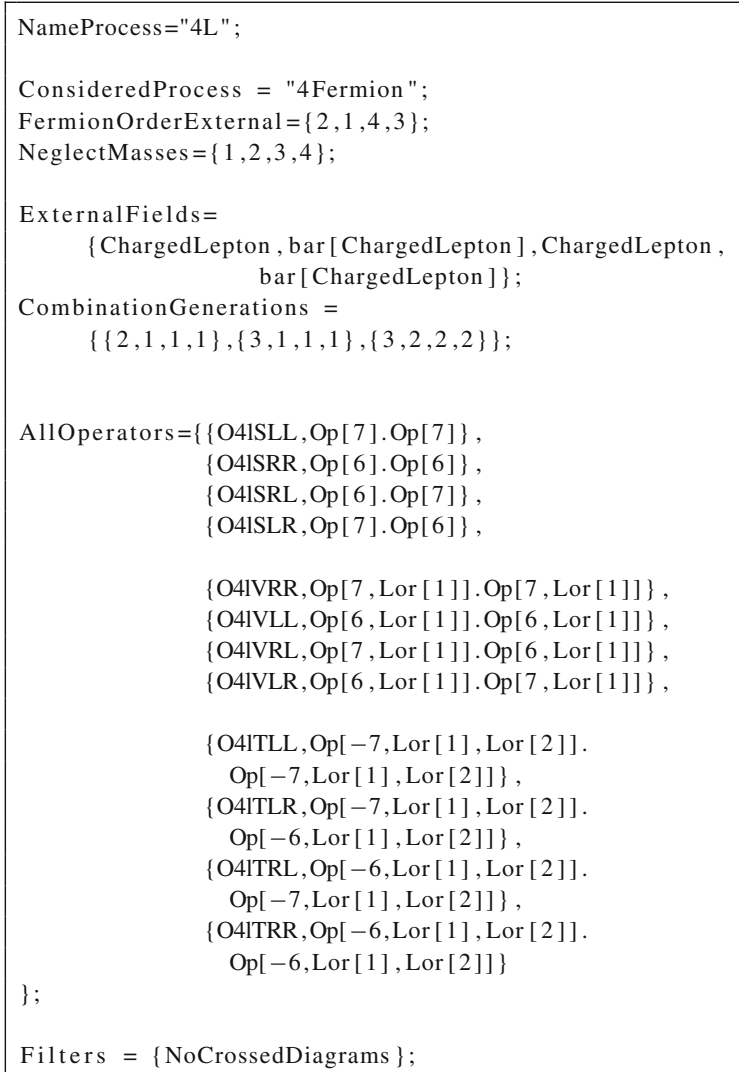

\begin{tabular}{lll}
\hline$(\bar{d} \Gamma u)\left(\bar{\ell} \Gamma^{\prime} \nu\right)$ & & \\
\hline Variable & Operator & Name \\
\hline OdulvVLL & $\left(\bar{d} \gamma_{\mu} P_{L} u\right)\left(\bar{\ell} \gamma^{\mu} P_{L} v\right)$ & $G_{L L}^{V}$ \\
OdulvVRR & $\left(\bar{d} \gamma_{\mu} P_{R} u\right)\left(\bar{\ell} \gamma^{\mu} P_{R} v\right)$ & $G_{R R}^{V}$ \\
OdulvVLR & $\left(\bar{d} \gamma_{\mu} P_{L} u\right)\left(\bar{\ell} \gamma^{\mu} P_{R} v\right)$ & $G_{L R}^{V}$ \\
OdulvVRL & $\left(\bar{d} \gamma_{\mu} P_{R} u\right)\left(\bar{\ell} \gamma^{\mu} P_{L} v\right)$ & $G_{R L}^{V}$ \\
OdulvSLL & $\left(\bar{d} P_{L} u\right)\left(\bar{\ell} P_{L} v\right)$ & $G_{L L}^{S}$ \\
OdulvSRR & $\left(\bar{d} P_{R} u\right)\left(\bar{\ell} P_{R} v\right)$ & $G_{R R}^{S}$ \\
OdulvSLR & $\left(\bar{d} P_{L} u\right)\left(\bar{\ell} P_{R} v\right)$ & $G_{L R}^{S}$ \\
OdulvSRL & $\left(\bar{d} P_{R} u\right)\left(\bar{\ell} P_{L} v\right)$ & $G_{R L}^{S}$ \\
\hline
\end{tabular}

Listing 17 du_lv.m

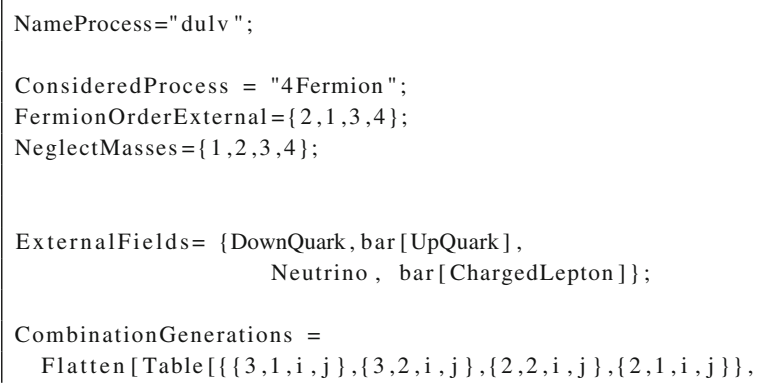




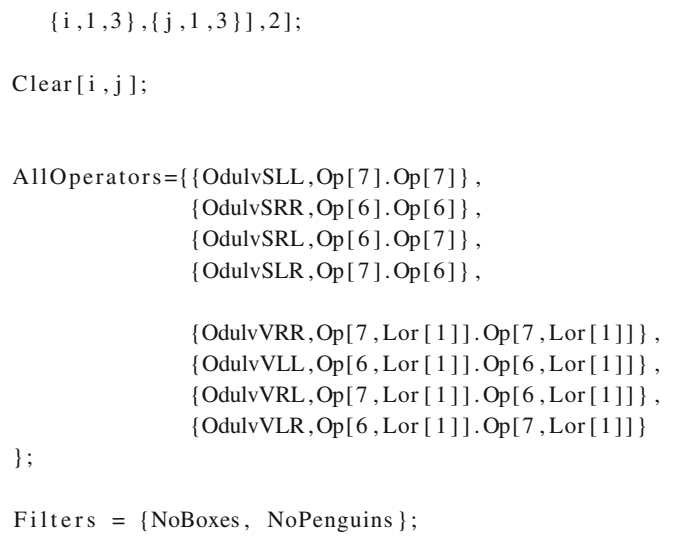

\section{Appendix C: Application: flavor observables implemen- ted in SARAH}

\section{C.1 Lepton flavor observables}

Lepton flavor violation in the SM or MSSM without neutrino masses vanishes exactly. Even adding Dirac neutrino masses to the SM predicts LFV rates which are far beyond the experimental reach. However, many extensions of the SM can introduce new sources for LFV of a size which is testable nowadays. The best-known examples are SUSY and nonSUSY models with high- or low-scale seesaw mechanism, models with vector-like leptons and SUSY models with $R$ parity violation, see for instance Refs. [32,42,58-89].

We discuss in the following the implementation of the most important LFV observables in SARAH and SPheno using the previously defined operators which are calculated by SPheno.

\section{C.1.1 $\ell_{\alpha} \rightarrow \ell_{\beta} \gamma$}

The decay width is given by [42]

$$
\Gamma\left(\ell_{\alpha} \rightarrow \ell_{\beta} \gamma\right)=\frac{\alpha m_{\ell_{\alpha}}^{5}}{4}\left(\left|K_{2}^{L}\right|^{2}+\left|K_{2}^{R}\right|^{2}\right),
$$

where $\alpha$ is the fine structure constant and the dipole Wilson coefficients $K_{2}^{L, R}$ are defined in Eq. (A.3).

\section{Listing 18 LLgGamma.m}

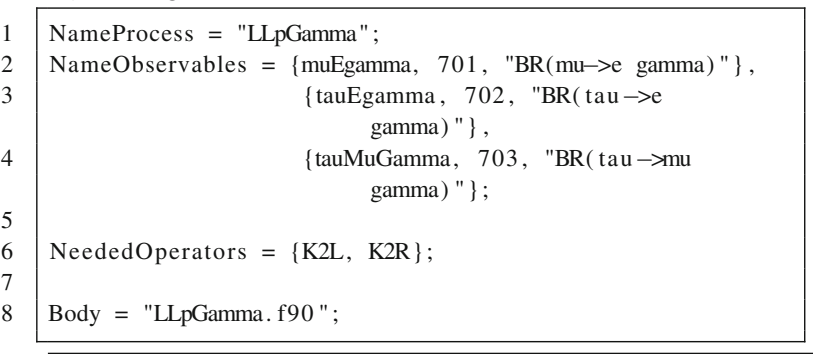

\section{Listing 19 LLgGamma.f90}

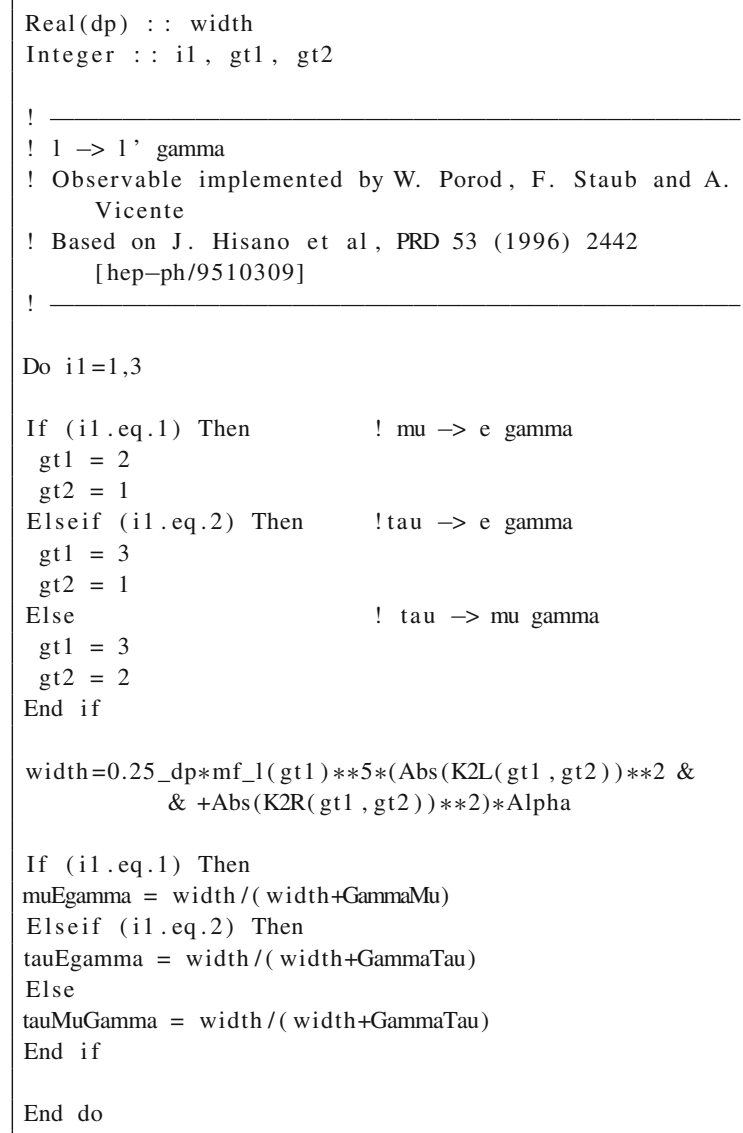

\section{C.1.2 $\ell_{\alpha} \rightarrow 3 \ell_{\beta}$}

The decay width is given by

$$
\begin{aligned}
\Gamma & \left(\ell_{\alpha} \rightarrow 3 \ell_{\beta}\right)=\frac{m_{\ell_{\alpha}}^{5}}{512 \pi^{3}}\left[e^{4}\left(\left|K_{2}^{L}\right|^{2}+\left|K_{2}^{R}\right|^{2}\right)\right. \\
& \times\left(\frac{16}{3} \log \frac{m_{\ell_{\alpha}}}{m_{\ell_{\beta}}}-\frac{22}{3}\right) \\
& +\frac{1}{24}\left(\left|A_{L L}^{S}\right|^{2}+\left|A_{R R}^{S}\right|^{2}\right)+\frac{1}{12}\left(\left|A_{L R}^{S}\right|^{2}+\left|A_{R L}^{S}\right|^{2}\right) \\
& +\frac{2}{3}\left(\left|\hat{A}_{L L}^{V}\right|^{2}+\left|\hat{A}_{R R}^{V}\right|^{2}\right)+\frac{1}{3}\left(\left|\hat{A}_{L R}^{V}\right|^{2}+\left|\hat{A}_{R L}^{V}\right|^{2}\right) \\
& +6\left(\left|A_{L L}^{T}\right|^{2}+\left|A_{R T}^{T}\right|^{2}\right) \\
& +\frac{e^{2}}{3}\left(K_{2}^{L} A_{R L}^{S *}+K_{2}^{R} A_{L R}^{S *}+\text { c.c. }\right) \\
& -\frac{2 e^{2}}{3}\left(K_{2}^{L} \hat{A}_{R L}^{V *}+K_{2}^{R} \hat{A}_{L R}^{V *}+\text { c.c. }\right) \\
& -\frac{4 e^{2}}{3}\left(K_{2}^{L} \hat{A}_{R R}^{V *}+K_{2}^{R} \hat{A}_{L L}^{V *}+\text { c.c. }\right)
\end{aligned}
$$




$$
\begin{aligned}
& -\frac{1}{2}\left(A_{L L}^{S} A_{L L}^{T *}+A_{R R}^{S} A_{R R}^{T *}+\text { c.c. }\right) \\
& \left.-\frac{1}{6}\left(A_{L R}^{S} \hat{A}_{L R}^{V *}+A_{R L}^{S} \hat{A}_{R L}^{V *}+\text { c.c. }\right)\right] .
\end{aligned}
$$

Here we have defined

$$
\hat{A}_{X Y}^{V}=A_{X Y}^{V}+e^{2} K_{1}^{X} \quad(X, Y=L, R) .
$$

The mass of the leptons in the final state has been neglected in this formula, with the exception of the dipole terms $K_{2}^{L, R}$, where an infrared divergence would otherwise occur due to the massless photon propagator. Equation (C.19) is in agreement with [58], but also includes the coefficients $A_{L R}^{S}$ and $A_{R L}^{S}$.

\section{Listing 20 Lto3Lp.m}

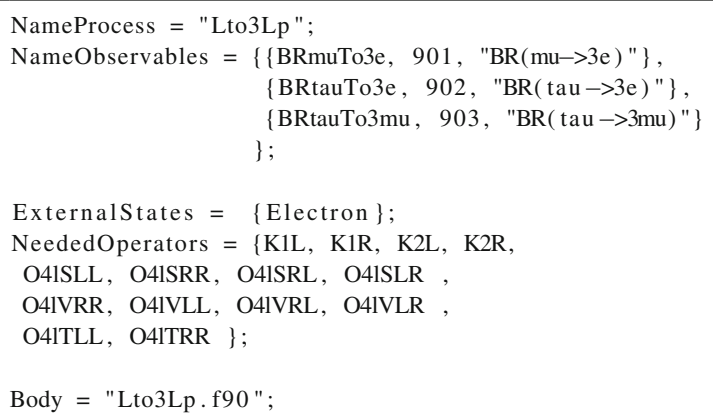

\section{Listing 21 Lto3Lp.f90}

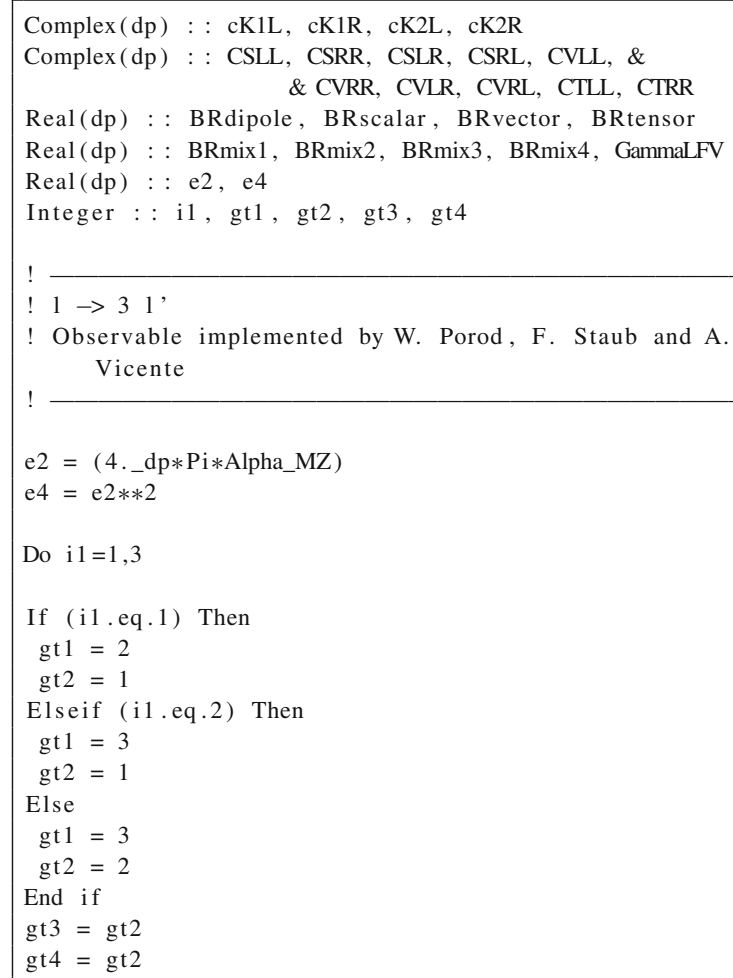

$\mathrm{cK} 2 \mathrm{~L}=\mathrm{K} 2 \mathrm{~L}(\mathrm{gt} 1, \mathrm{gt} 2)$

$36 \mathrm{cK} 2 \mathrm{R}=\mathrm{K} 2 \mathrm{R}(\mathrm{gt} 1, \mathrm{gt} 2)$

38 CSLL $=$ O41SLL $(\mathrm{gt} 1, \mathrm{gt} 2, \mathrm{gt} 3, \mathrm{gt} 4)$

$\mathrm{CSRR}=\mathrm{O} 4 \mathrm{lSRR}(\mathrm{gt} 1, \mathrm{gt} 2, \mathrm{gt} 3, \mathrm{gt} 4)$

CSLR $=$ O4lSLR $($ gt 1, gt $2, g t 3, g t 4)$

$\mathrm{CSRL}=\mathrm{O} 4 \mathrm{ISRL}(\mathrm{gt} 1, \mathrm{gt} 2, \mathrm{gt} 3, \mathrm{gt} 4)$

CVLL $=$ O4lVLL $(g t 1, g t 2, g t 3, g t 4)$

CVRR $=$ O4lVRR $($ gt 1, gt 2, gt 3, gt 4$)$

CVLR $=$ O4lVLR $(\mathrm{gt} 1, \mathrm{gt} 2, \mathrm{gt} 3, \mathrm{gt} 4)$

CVRL $=$ O4lVRL $(\mathrm{gt} 1, \mathrm{gt} 2, \mathrm{gt} 3, \mathrm{gt} 4)$

$\mathrm{CVLL}=\mathrm{CVLL}+\mathrm{e} 2 * \mathrm{CK} 1 \mathrm{~L}$

CVRR $=$ CVRR $+\mathrm{e} 2 * \mathrm{CK} 1 \mathrm{R}$

CVLR $=$ CVLR $+\mathrm{e} 2 * \mathrm{CK} 1 \mathrm{~L}$

CVRL $=$ CVRL $+\mathrm{e} 2 * \mathrm{CK} 1 \mathrm{R}$

CTLL $=$ O4ITLL $(\mathrm{gt} 1, \mathrm{gt} 2, \mathrm{gt} 3, \mathrm{gt} 4)$

$\mathrm{CTRR}=\mathrm{O} 4 \mathrm{ITRR}(\mathrm{gt} 1, \mathrm{gt} 2, \mathrm{gt} 3, \mathrm{gt} 4)$

! Photonic dipole contributions

BRdipole $=(\operatorname{Abs}(\mathrm{cK} 2 \mathrm{~L}) * * 2+\operatorname{Abs}(\mathrm{cK} 2 \mathrm{R}) * * 2) \&$

$\& *\left(16 . \_d p * \log \left(\mathrm{mf} \_1(\mathrm{gt} 1) / \mathrm{mf} \_1(\mathrm{gt} 2)\right)-22\right.$.ddp $) / 3$.dp

! Scalar contributions

BRscalar $=(\operatorname{Abs}(\mathrm{CSLL}) * * 2+\mathrm{Abs}(\mathrm{CSRR}) * * 2) / 24 . \_\mathrm{dp} \&$

$\&+(\operatorname{Abs}(\mathrm{CSLR}) * * 2+\operatorname{Abs}(\mathrm{CSRL}) * * 2) / 12 . \mathrm{dp}$

! Vector contributions

BRvector $=2$._dp $*(\operatorname{Abs}(\mathrm{CVLL}) * * 2+\mathrm{Abs}(\mathrm{CVRR}) * * 2) / 3 . \_\mathrm{dp} \&$

$\&+(\operatorname{Abs}(\mathrm{CVLR}) * * 2+\operatorname{Abs}(\mathrm{CVRL}) * * 2) / 3 . \mathrm{dp}$

! Tensor contributions

BRtensor $=6 . \_\mathrm{dp} *(\mathrm{Abs}(\mathrm{CTLL}) * * 2+\operatorname{Abs}(\mathrm{CTRR}) * * 2)$

! Mix: dipole x scalar

BRmix $1=2$. dp $/ 3 . \_d p * \operatorname{Real}(\mathrm{cK} 2 \mathrm{~L} * \operatorname{Conjg}(\mathrm{CSRL})+$ cK2R*Conjg (CSLR), dp)

! Mix: dipole $\mathrm{x}$ vector

BRmix $2=-4 . \_d p / 3 . \_d p * \operatorname{Real}(\mathrm{cK} 2 \mathrm{~L} * \operatorname{Conjg}(\mathrm{CVRL})+$ $\mathrm{cK} 2 \mathrm{R} *$ Conjg $(\mathrm{CVLR}), \mathrm{dp}) \&$ \& -8._dp/3._dp $* \operatorname{Real}(\mathrm{cK} 2 \mathrm{~L} * \operatorname{Conjg}(\mathrm{CVRR})+$ $\mathrm{cK} 2 \mathrm{R} *$ Conjg $(\mathrm{CVLL}), \mathrm{dp})$

! Mix: scalar $x$ vector

BRmix $3=-1 . \_d p / 3 . \_d p * \operatorname{Real}(\operatorname{CSLR} * \operatorname{Conjg}(\mathrm{CVLR})+$ CSRL $*$ Conjg (CVRL), dp)

! Mix: scalar $x$ tensor

BRmix4 $=-1$. dp $* \operatorname{Real}(\operatorname{CSLL} * \operatorname{Conjg}(\mathrm{CTLL})+$ CSRR $*$ Conjg $(\mathrm{CTRR}), \mathrm{dp})$

GammaLFV $=00512$ pi3 $*$ mf_l $($ gt 1$) * * 5 * \&$ $\&($ e $4 *$ BRdipole + BRscalar + BRvector + BRtensor \& $\&+\mathrm{e} 2 * \mathrm{BRmix} 1+\mathrm{e} 2 * \mathrm{BRmix} 2+\mathrm{BRmix} 3+\mathrm{BRmix} 4)$

!taking alpha $(\mathrm{Q}=0)$ instead of alpha $\left(\mathrm{m} \_\mathrm{Z}\right)$ as this contains most of the

!running of the Wilson coefficients

If (i1 .Eq. 1) Then

BRmuTo3e $=$ GammaLFV $/$ GammaMu

Else If (i1.Eq.2) Then

BRtauTo3e $=$ GammaLFV $/$ GammaTau

Else

BRtauTo3mu=GammaLFV/GammaTau 
99 End If

100 End do

\section{C.1.3 Coherent $\boldsymbol{\mu}-\boldsymbol{e}$ conversion in nuclei}

The conversion rate, relative to the the muon capture rate, can be expressed as $[90,91]$

$$
\begin{aligned}
\mathrm{CR}(\mu-e, \text { Nucleus })= & \frac{p_{e} E_{e} m_{\mu}^{3} G_{F}^{2} \alpha^{3} Z_{\mathrm{eff}}^{4} F_{p}^{2}}{8 \pi^{2} Z} \\
& \times\left\{\mid(Z+N)\left(g_{L V}^{(0)}+g_{L S}^{(0)}\right)\right. \\
& +\left.(Z-N)\left(g_{L V}^{(1)}+g_{L S}^{(1)}\right)\right|^{2} \\
& +\mid(Z+N)\left(g_{R V}^{(0)}+g_{R S}^{(0)}\right) \\
& \left.+\left.(Z-N)\left(g_{R V}^{(1)}+g_{R S}^{(1)}\right)\right|^{2}\right\} \frac{1}{\Gamma_{\text {capt }}} .
\end{aligned}
$$

$Z$ and $N$ are the number of protons and neutrons in the nucleus and $Z_{\text {eff }}$ is the effective atomic charge [92]. Similarly, $G_{F}$ is the Fermi constant, $F_{p}$ is the nuclear matrix element and $\Gamma_{\text {capt }}$ represents the total muon capture rate. $\alpha$ is the fine structure constant, $p_{e}$ and $E_{e}\left(\simeq m_{\mu}\right.$ in the numerical evaluation) are the momentum and energy of the electron and $m_{\mu}$ is the muon mass. In the above, $g_{X K}^{(0)}$ and $g_{X K}^{(1)}$ (with $X=L, R$ and $K=S, V$ ) can be written in terms of effective couplings at the quark level as

$g_{X K}^{(0)}=\frac{1}{2} \sum_{q=u, d, s}\left(g_{X K(q)} G_{K}^{(q, p)}+g_{X K(q)} G_{K}^{(q, n)}\right)$,

$g_{X K}^{(1)}=\frac{1}{2} \sum_{q=u, d, s}\left(g_{X K(q)} G_{K}^{(q, p)}-g_{X K(q)} G_{K}^{(q, n)}\right)$.

For coherent $\mu-e$ conversion in nuclei, only scalar $(S)$ and vector $(V)$ couplings contribute. Furthermore, sizable contributions are expected only from the $u, d, s$ quark flavors. The numerical values of the relevant $G_{K}$ factors are [90,93]

$$
\begin{aligned}
& G_{V}^{(u, p)}=G_{V}^{(d, n)}=2 ; \quad G_{V}^{(d, p)}=G_{V}^{(u, n)}=1 ; \\
& G_{S}^{(u, p)}=G_{S}^{(d, n)}=5.1 ; \quad G_{S}^{(d, p)}=G_{S}^{(u, n)}=4.3 ; \\
& G_{S}^{(s, p)}=G_{S}^{(s, n)}=2.5 .
\end{aligned}
$$

Finally, the $g_{X K(q)}$ coefficients can be written in terms of the Wilson coefficients in Eqs. (A.3), (A.8) and (A.9) as

$g_{L V(q)}=\frac{\sqrt{2}}{G_{F}}\left[e^{2} Q_{q}\left(K_{1}^{L}-K_{2}^{R}\right)-\frac{1}{2}\left(C_{\ell \ell q q}^{V L L}+C_{\ell \ell q q}^{V L R}\right)\right]$
$g_{R V(q)}=\left.g_{L V(q)}\right|_{L \rightarrow R}$

$g_{L S(q)}=-\frac{\sqrt{2}}{G_{F}} \frac{1}{2}\left(C_{\ell \ell q q}^{S L L}+C_{\ell \ell q q}^{S L R}\right)$

$g_{R S(q)}=\left.g_{L S(q)}\right|_{L \rightarrow R}$.

Here $Q_{q}$ is the quark electric charge $\left(Q_{d}=-1 / 3, Q_{u}=\right.$ $2 / 3$ ) and $C_{\ell \ell q q}^{I X K}=B_{X Y}^{K}\left(C_{X Y}^{K}\right)$ for d-quarks (u-quarks), with $X=L, R$ and $K=S, V$.

Listing 22 MuEconversion.m

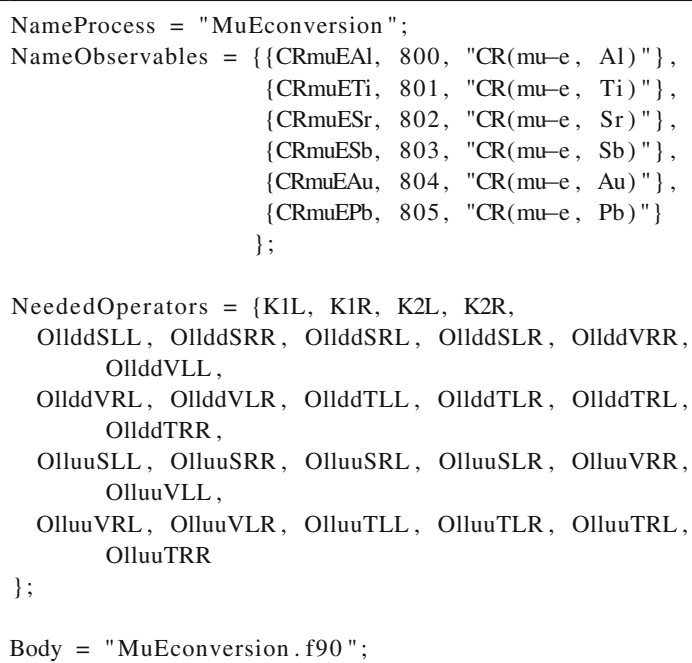

\section{Listing 23 MuEconversion.f90}

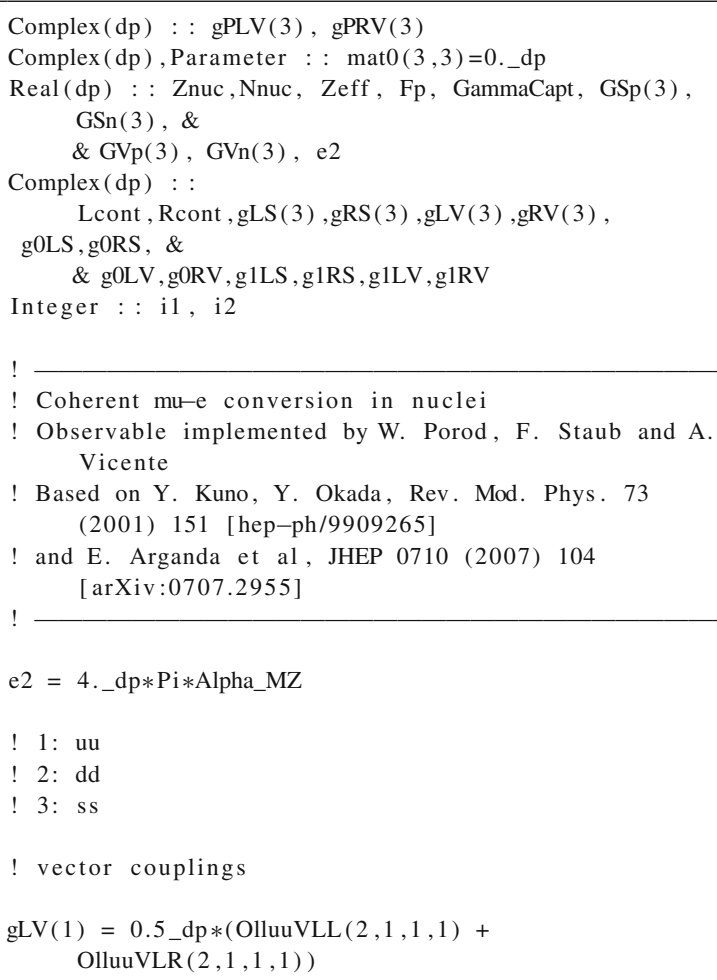




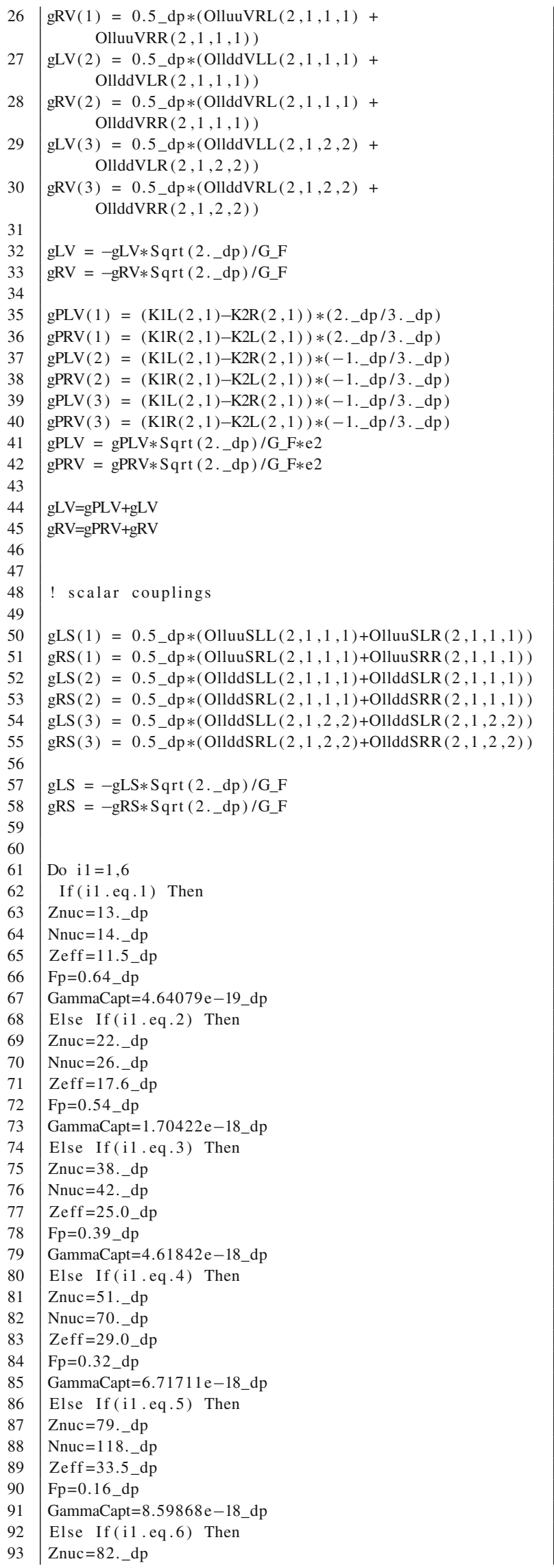

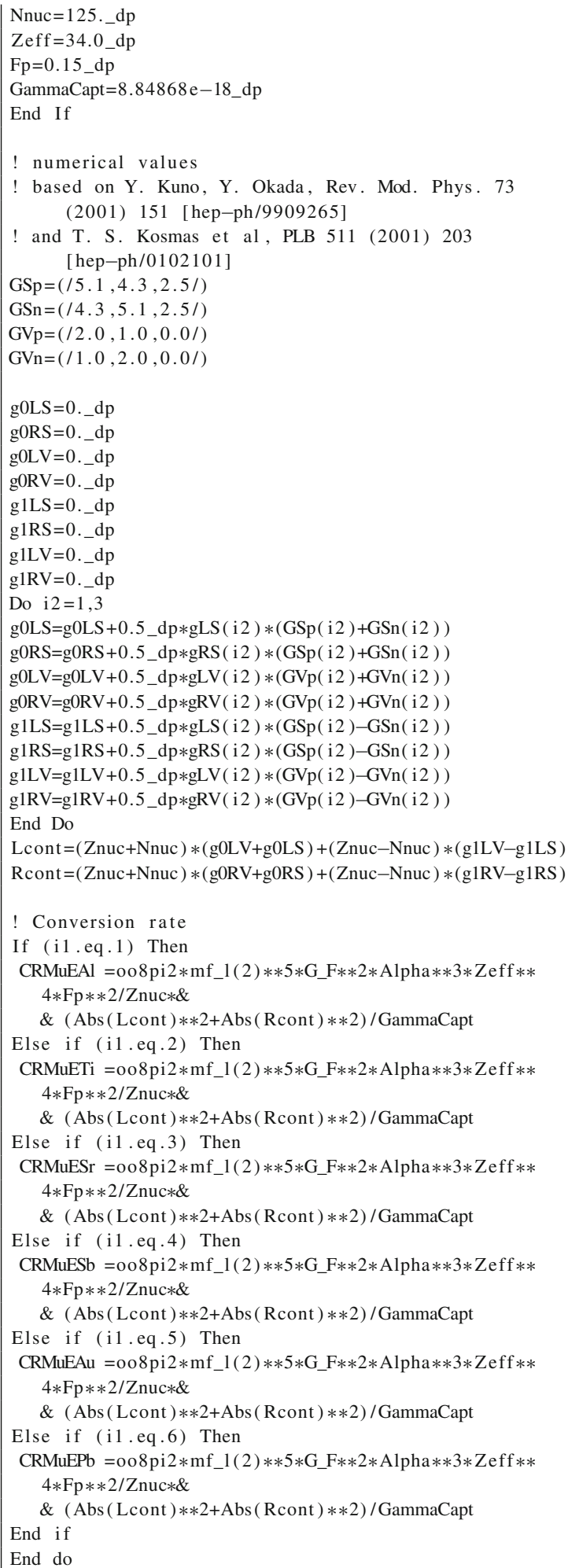

\section{C.1.4 $\tau \rightarrow P \ell$}

Our analytical expressions for $\tau \rightarrow P \ell$, where $\ell=e, \mu$ and $P$ is a pseudoscalar meson, generalize the results in [94]. The decay width is given by 
$\Gamma(\tau \rightarrow \ell P)=\frac{1}{4 \pi} \frac{\lambda^{1 / 2}\left(m_{\tau}^{2}, m_{\ell}^{2}, m_{P}^{2}\right)}{m_{\tau}^{2}} \frac{1}{2} \sum_{i, f}\left|\mathcal{M}_{\tau \ell P}\right|^{2}$,

where the averaged squared amplitude can be written as

$$
\begin{aligned}
& \frac{1}{2} \sum_{i, f}\left|\mathcal{M}_{\tau \ell P}\right|^{2}=\frac{1}{4 m_{\tau}} \sum_{I, J=S, V}\left[2 m_{\tau} m_{\ell}\left(a_{P}^{I} a_{P}^{J *}-b_{P}^{I} b_{P}^{J *}\right)\right. \\
& \left.\quad+\left(m_{\tau}^{2}+m_{\ell}^{2}-m_{P}^{2}\right)\left(a_{P}^{I} a_{P}^{J *}+b_{P}^{I} b_{P}^{J *}\right)\right] .
\end{aligned}
$$

The coefficients $a_{P}^{S, V}$ and $b_{P}^{S, V}$ can be expressed in terms of the Wilson coefficients in Eqs. (A.8) and (A.9) as

$$
\begin{aligned}
a_{P}^{S}= & \frac{1}{2} f_{\pi} \sum_{X=L, R}\left[\frac{D_{X}^{d}(P)}{m_{d}}\left(B_{L X}^{S}+B_{R X}^{S}\right)\right. \\
& \left.+\frac{D_{X}^{u}(P)}{m_{u}}\left(C_{L X}^{S}+C_{R X}^{S}\right)\right] \\
b_{P}^{S}= & \frac{1}{2} f_{\pi} \sum_{X=L, R}\left[\frac{D_{X}^{d}(P)}{m_{d}}\left(B_{R X}^{S}-B_{L X}^{S}\right)\right. \\
& \left.+\frac{D_{X}^{u}(P)}{m_{u}}\left(C_{R X}^{S}-C_{L X}^{S}\right)\right] \\
a_{P}^{V}= & \frac{1}{4} f_{\pi} C(P)\left(m_{\tau}-m_{\ell}\right)\left[-B_{L L}^{V}+B_{L R}^{V}-B_{R L}^{V}+B_{R R}^{V}\right. \\
& \left.+C_{L L}^{V}-C_{L R}^{V}+C_{R L}^{V}-C_{R R}^{V}\right] \\
b_{P}^{V}= & \frac{1}{4} f_{\pi} C(P)\left(m_{\tau}+m_{\ell}\right)\left[-B_{L L}^{V}+B_{L R}^{V}+B_{R L}^{V}-B_{R R}^{V}\right. \\
& \left.+C_{L L}^{V}-C_{L R}^{V}-C_{R L}^{V}+C_{R R}^{V}\right] .
\end{aligned}
$$

In these expressions $m_{d}$ and $m_{u}$ are the down- and up-quark masses, respectively, $f_{\pi}$ is the pion decay constant and the coefficients $C(P), D_{L, R}^{d, u}(P)$ take different forms for each pseudoscalar meson $P$ [94]. For $P=\pi$ one has

$C(\pi)=1$

$D_{L}^{d}(\pi)=-\frac{m_{\pi}^{2}}{4}$

$D_{L}^{u}(\pi)=\frac{m_{\pi}^{2}}{4}$,

for $P=\eta$

$C(\eta)=\frac{1}{\sqrt{6}}\left(\sin \theta_{\eta}+\sqrt{2} \cos \theta_{\eta}\right)$
$D_{L}^{d}(\eta)=\frac{1}{4 \sqrt{3}}\left[\left(3 m_{\pi}^{2}-4 m_{K}^{2}\right) \cos \theta_{\eta}-2 \sqrt{2} m_{K}^{2} \sin \theta_{\eta}\right]$

$D_{L}^{u}(\eta)=\frac{1}{4 \sqrt{3}} m_{\pi}^{2}\left(\cos \theta_{\eta}-\sqrt{2} \sin \theta_{\eta}\right)$,

and for $P=\eta^{\prime}$

$C\left(\eta^{\prime}\right)=\frac{1}{\sqrt{6}}\left(\sqrt{2} \sin \theta_{\eta}-\cos \theta_{\eta}\right)$

$D_{L}^{d}\left(\eta^{\prime}\right)=\frac{1}{4 \sqrt{3}}\left[\left(3 m_{\pi}^{2}-4 m_{K}^{2}\right) \sin \theta_{\eta}+2 \sqrt{2} m_{K}^{2} \cos \theta_{\eta}\right]$

$D_{L}^{u}\left(\eta^{\prime}\right)=\frac{1}{4 \sqrt{3}} m_{\pi}^{2}\left(\sin \theta_{\eta}+\sqrt{2} \cos \theta_{\eta}\right)$.

Here $m_{\pi}$ and $m_{K}$ are the masses of the neutral pion and Kaon, respectively, and $\theta_{\eta}$ is the $\eta-\eta^{\prime}$ mixing angle. In addition, $D_{R}^{d, u}(P)=-\left(D_{L}^{d, u}(P)\right)^{*}$.

Notice that the Wilson coefficients in Eq. (C.33) include all pseudoscalar and axial contributions to $\tau \rightarrow \ell P$. Therefore, this goes beyond some well-known results in the literature, see for example [94,95], where box contributions were neglected.

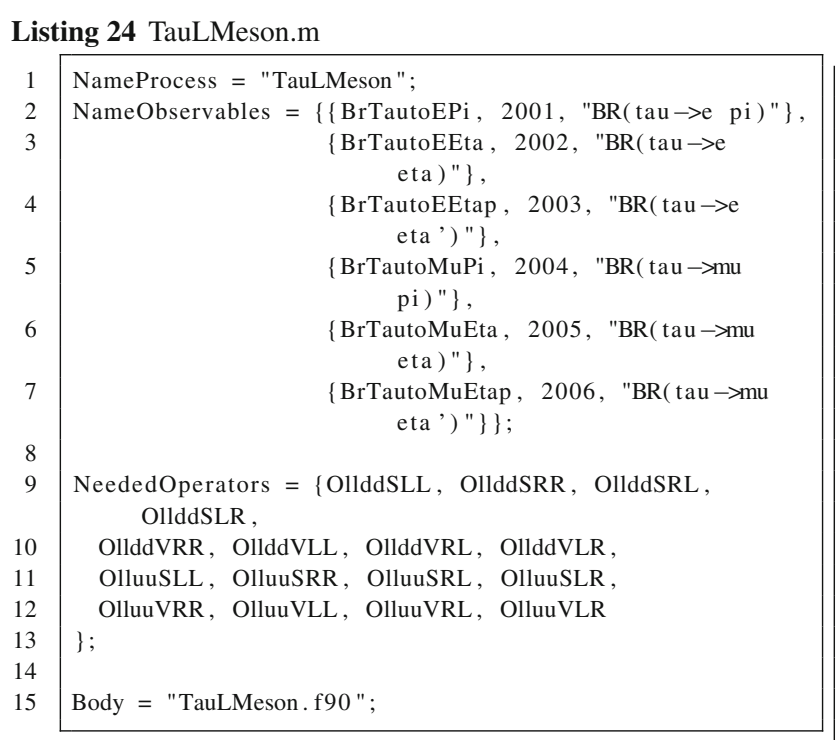

\section{Listing 25 TauLMeson.f90}

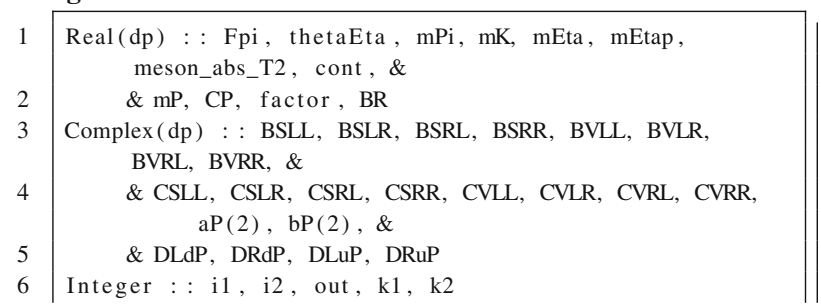




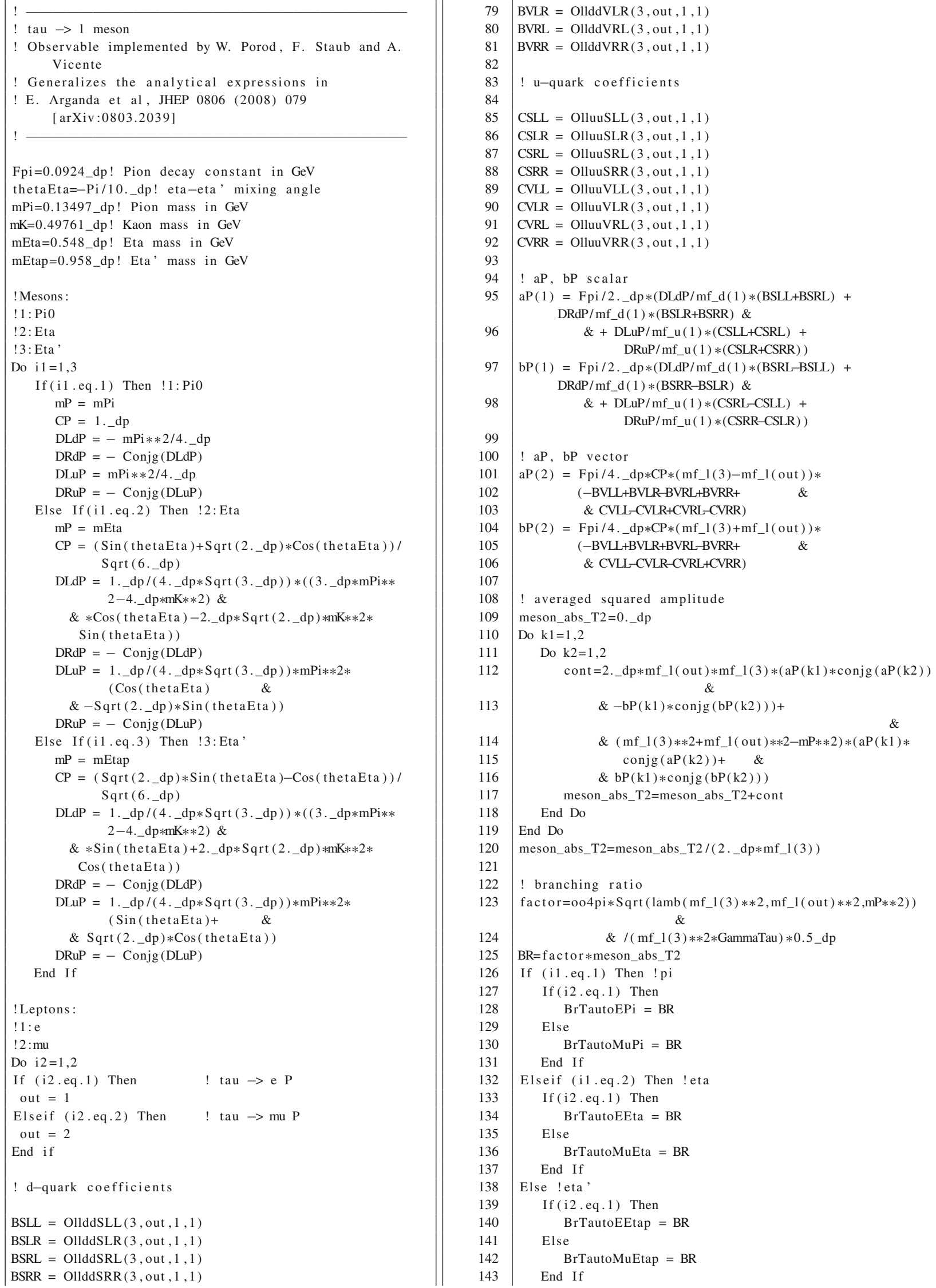

BVLR $=\operatorname{OllddVLR}(3$, out $, 1,1)$

BVRL $=\operatorname{OllddVRL}(3$, out $, 1,1)$

BVRR $=\operatorname{OllddVRR}(3$, out $, 1,1)$

! u-quark coefficients

CSLL $=$ OlluuSLL $(3$, out $, 1,1)$

$\operatorname{CSLR}=\operatorname{OlluuSLR}(3$, out $, 1,1)$

CSRL $=$ OlluuSRL $(3$, out $, 1,1)$

CSRR $=\operatorname{OlluuSRR}(3$, out $, 1,1)$

CVLL $=\operatorname{OlluuVLL}(3$, out $, 1,1)$

CVLR $=$ OlluuVLR $(3$, out $, 1,1)$

CVRL $=\operatorname{OlluuVRL}(3$, out $, 1,1)$

CVRR $=$ OlluuVRR $(3$, out $, 1,1)$

! aP, bP scalar

$\mathrm{aP}(1)=\mathrm{Fpi} / 2 . \_\mathrm{dp} *\left(\mathrm{DLdP} / \mathrm{mf} \_\mathrm{d}(1) *(\mathrm{BSLL}+\mathrm{BSRL})+\right.$ $\mathrm{DRdP} / \mathrm{mf} \_\mathrm{d}(1) *(\mathrm{BSLR}+\mathrm{BSRR}) \&$

$\&+\mathrm{DLuP} / \mathrm{mf} \_\mathrm{u}(1) *(\mathrm{CSLL}+\mathrm{CSRL})+$ $\left.\mathrm{DRuP} / \mathrm{mf} \_\mathrm{u}(1) *(\mathrm{CSLR}+\mathrm{CSRR})\right)$

$\mathrm{bP}(1)=\mathrm{Fpi} / 2 . \mathrm{dp} *\left(\mathrm{DLdP} / \mathrm{mf} \_\mathrm{d}(1) *(\mathrm{BSRL}-\mathrm{BSLL})+\right.$ $\mathrm{DRdP} / \mathrm{mf} \_\mathrm{d}(1) *(\mathrm{BSRR}-\mathrm{BSLR}) \&$

$\&+\mathrm{DLuP} / \mathrm{mf} \mathrm{u}(1) *(\mathrm{CSRL}-\mathrm{CSLL})+$ $\left.\mathrm{DRuP} / \mathrm{mf} \_\mathrm{u}(1) *(\mathrm{CSRR}-\mathrm{CSLR})\right)$

! aP, bP vector

$\mathrm{aP}(2)=\mathrm{Fpi} / 4 . \_\mathrm{dp} * \mathrm{CP} *\left(\mathrm{mf} \_1(3)-\mathrm{mf} \_1(\right.$ out $\left.)\right) *$ $(-\mathrm{BVLL+BVLR}-\mathrm{BVRL}+\mathrm{BVRR}+\quad \&$ \& CVLL-CVLR+CVRL-CVRR)

$\mathrm{bP}(2)=\mathrm{Fpi} / 4$. dp $* \mathrm{CP} *\left(\mathrm{mf} \_1(3)+\mathrm{mf} \_1(\right.$ out $\left.)\right) *$ $(-\mathrm{BVL}+\mathrm{BVLR+BVRL-BVRR+ \&} \mathrm{\&}$ \& CVLL-CVLR-CVRL+CVRR)

! averaged squared amplitude

meson_abs_T2 $=0$._dp

Do $\mathrm{k} 1=1,2$

Do $\mathrm{k} 2=1,2$

cont $=2$._dp $*$ mf_1 (out $) *$ mf_1 $(3) *(\mathrm{aP}(\mathrm{k} 1) * \mathrm{conjg}(\mathrm{aP}(\mathrm{k} 2))$ $\&$

$$
\&-\mathrm{bP}(\mathrm{k} 1) * \operatorname{conjg}(\mathrm{bP}(\mathrm{k} 2)))+
$$

\& $\left(\mathrm{mf} \_1(3) * * 2+\mathrm{mf} \_1(\mathrm{out}) * * 2-\mathrm{mP} * * 2\right) *(\mathrm{aP}(\mathrm{k} 1) *$ $\operatorname{conjg}(\mathrm{aP}(\mathrm{k} 2))+\quad \&$

$\& \mathrm{bP}(\mathrm{k} 1) * \operatorname{conjg}(\mathrm{bP}(\mathrm{k} 2)))$

meson_abs_T2 $=$ meson_abs_T2 $2+$ cont

End Do

End Do

meson_abs_T2 $=$ meson_abs_T2/(2._dp $*$ mf_1(3))

! branching ratio

factor $=004$ pi $* \operatorname{Sqrt}\left(\operatorname{lamb}\left(\mathrm{mf} \_1(3) * * 2, \mathrm{mf} \_1(\mathrm{out}) * * 2, \mathrm{mP} * * 2\right)\right)$ \&

$\& /\left(\mathrm{mf} \_1(3) * * 2 * \mathrm{GammaTau}\right) * 0.5 \_\mathrm{dp}$

$\mathrm{BR}=\mathrm{f}$ a c t or $*$ meson_abs_T2

If (i1.eq.1) Then ! pi

If (i2.eq.1) Then

BrTautoEPi $=$ BR

Else

BrTautoMuPi $=\mathrm{BR}$

End If

E1seif (i1.eq.2) Then !eta

If (i2.eq.1) Then

BrTautoEEta $=\mathrm{BR}$

Else

BrTautoMuEta $=$ BR

End If

Else ! eta'

If (i2.eq. 1) Then

BrTautoEEtap $=$ BR

Else

BrTautoMuEtap $=$ BR

End If

BVLL $=\operatorname{OllddVLL}(3$, out $, 1,1)$ 


\begin{tabular}{l|l||}
144 & End if \\
145 & \\
146 & End Do \\
147 & End Do \\
148 & \\
149 & Contains \\
150 & \\
151 & Real $(\mathrm{dp})$ Function lamb $(\mathrm{x}, \mathrm{y}, \mathrm{z})$ \\
152 & Real $(\mathrm{dp})$, Intent $($ in $):: \mathrm{x}, \mathrm{y}, \mathrm{z}$ \\
153 & lamb=(x+y-z) $* 2-4 .+\mathrm{dp} * \mathrm{x} * \mathrm{y}$ \\
154 & End Function lamb \\
\end{tabular}

\section{C.1.5 $h \rightarrow \ell_{\alpha} \ell_{\beta}$}

The decay width is given by [96]

$$
\begin{aligned}
\Gamma\left(h \rightarrow \ell_{\alpha} \ell_{\beta}\right) \equiv & \Gamma\left(h \rightarrow \ell_{\alpha} \bar{\ell}_{\beta}\right)+\Gamma\left(h \rightarrow \bar{\ell}_{\alpha} \ell_{\beta}\right) \\
= & \frac{1}{16 \pi m_{h}}\left[\left(1-\left(\frac{m_{\ell_{\alpha}}+m_{\ell_{\beta}}}{m_{h}}\right)^{2}\right)\right. \\
& \left.\times\left(1-\left(\frac{m_{\ell_{\alpha}}-m_{\ell_{\beta}}}{m_{h}}\right)^{2}\right)\right]^{1 / 2} \\
& \times\left[\left(m_{h}^{2}-m_{\ell_{\alpha}}^{2}-m_{\ell_{\beta}}^{2}\right)\left(\left|S_{L}\right|^{2}+\left|S_{R}\right|^{2}\right)_{\alpha \beta}\right. \\
& \left.-4 m_{\ell_{\alpha}} m_{\ell_{\beta}} \operatorname{Re}\left(S_{L} S_{R}^{*}\right)_{\alpha \beta}\right]+(\alpha \leftrightarrow \beta)
\end{aligned}
$$

\section{Listing 26 hLLp.m}

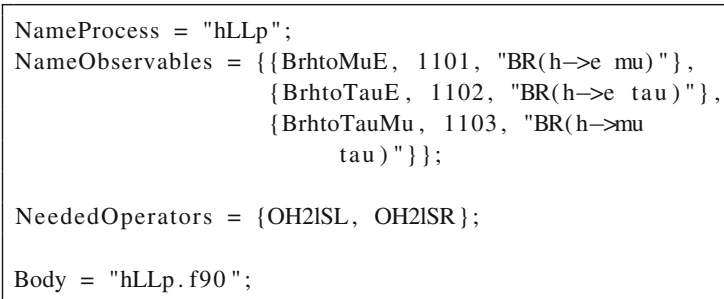

\section{Listing 27 hLLp.f90}

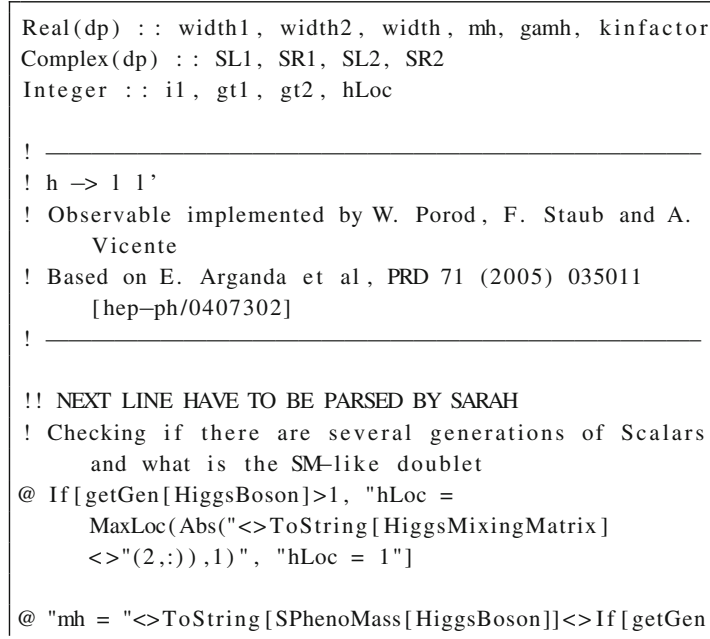

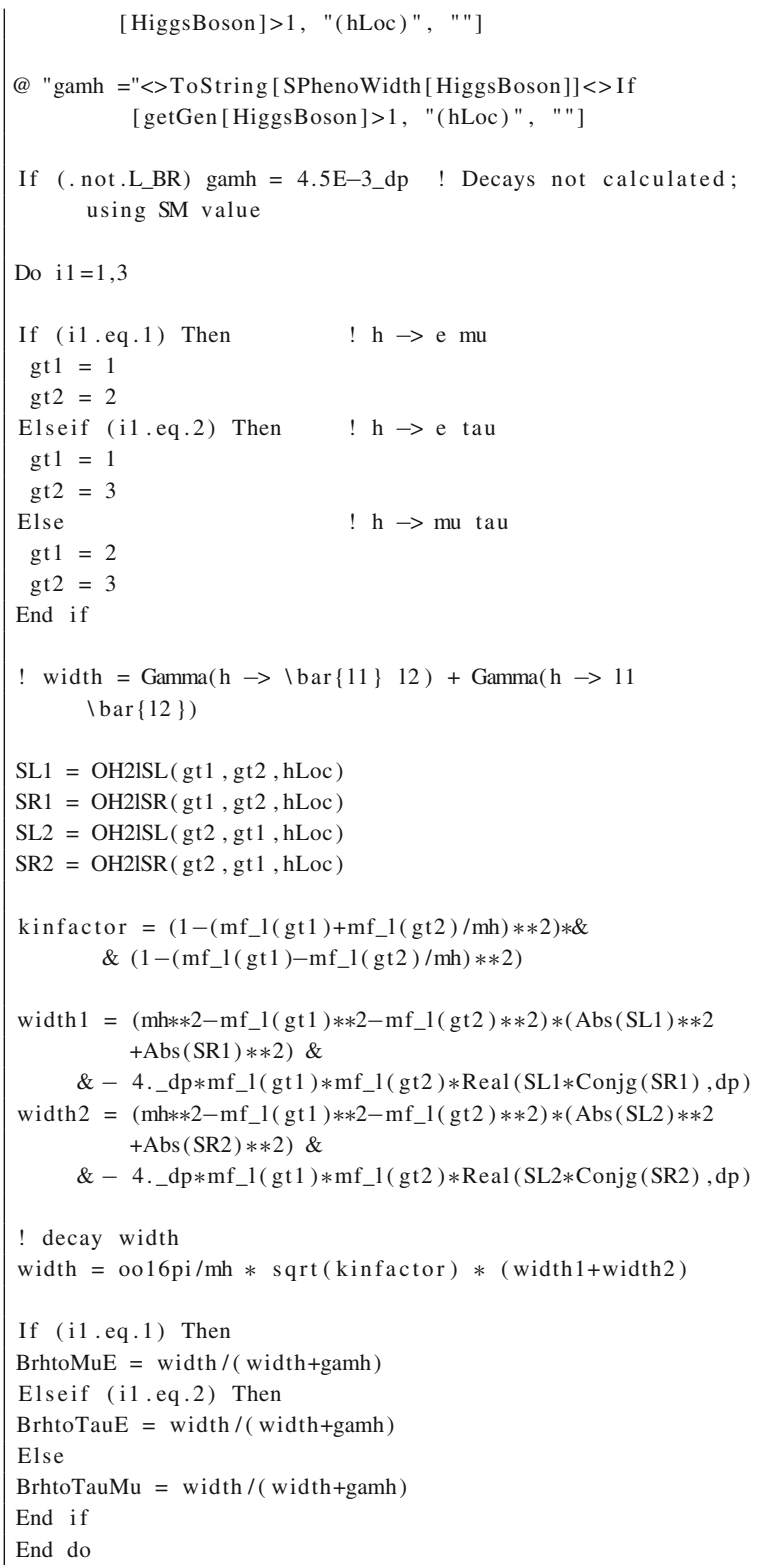

\section{C.1.6 $Z \rightarrow \ell_{\alpha} \ell_{\beta}$}

The decay width is given by [97]

$$
\begin{aligned}
\Gamma\left(Z \rightarrow \ell_{\alpha} \ell_{\beta}\right) \equiv & \Gamma\left(Z \rightarrow \ell_{\alpha} \bar{\ell}_{\beta}\right) \\
& +\Gamma\left(Z \rightarrow \bar{\ell}_{\alpha} \ell_{\beta}\right) \\
= & \frac{m_{Z}}{48 \pi}\left[2\left(\left|R_{1}^{L}\right|^{2}+\left|R_{1}^{R}\right|^{2}\right)\right. \\
& \left.+\frac{m_{Z}^{2}}{4}\left(\left|R_{2}^{L}\right|^{2}+\left|R_{2}^{R}\right|^{2}\right)\right],
\end{aligned}
$$

where the charged lepton masses have been neglected. 


\section{Listing 28 ZLLp.m}

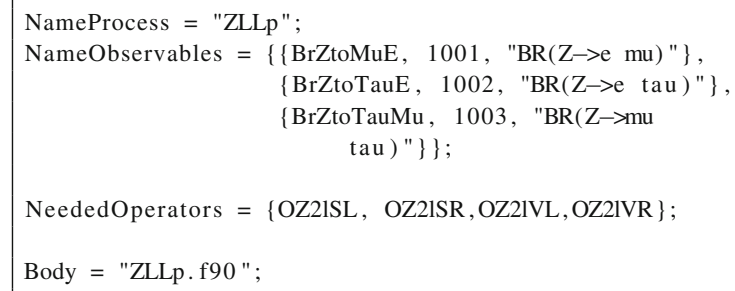

\section{Listing 29 ZLLp.f90}

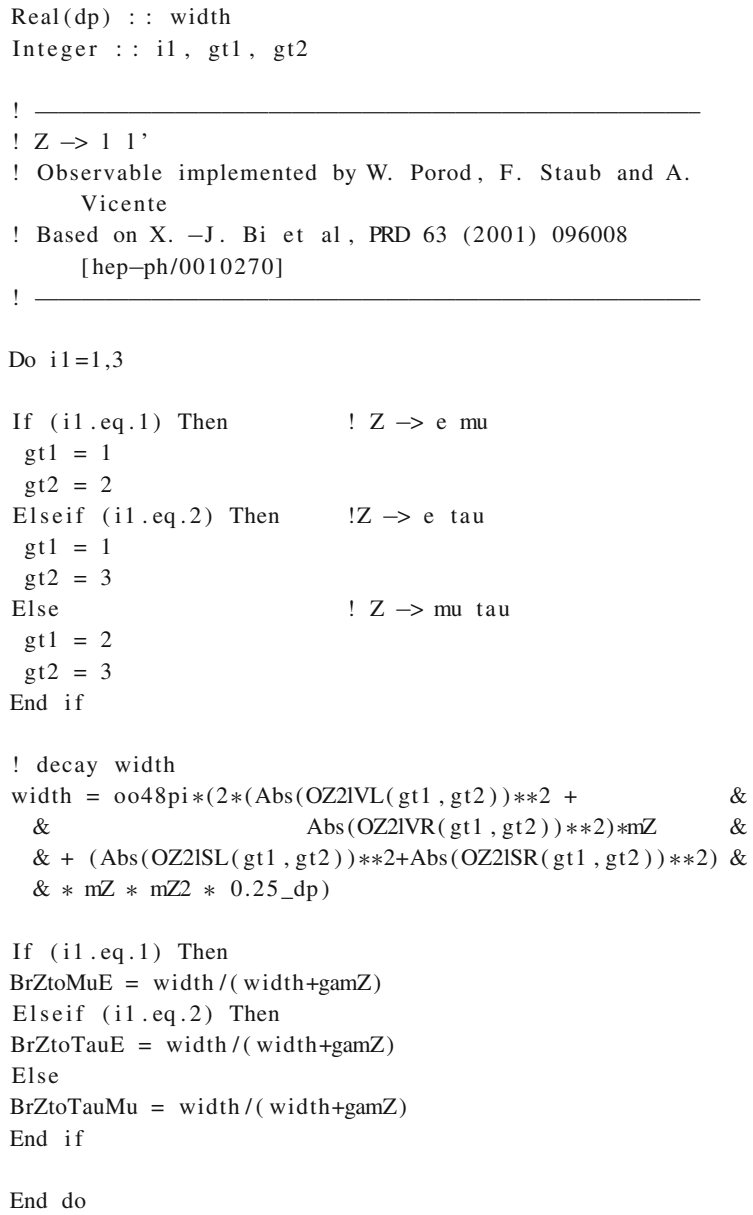

\section{C.2 Quark flavor observables}

QFV has been observed and its description in the SM due to the CKM matrix is well established. However, the large majority of BSM models causes additional contributions which have to be studied carefully, see for instance Refs. [98-122].

We give also here a description of the implementation of the different observables using the operators present in the SPheno output of SARAH.

$$
\text { C. } 3 B_{s, d}^{0} \rightarrow \ell^{+} \ell^{-}
$$

Our analytical results for $B_{s, d}^{0} \rightarrow \ell^{+} \ell^{-}$follow [103]. The $B^{0} \equiv B_{s, d}^{0}$ decay width to a pair of charged leptons can be written as

$$
\begin{gathered}
\Gamma\left(B^{0} \rightarrow \ell_{\alpha}^{+} \ell_{\beta}^{-}\right)=\frac{\mid \mathcal{M}_{\left.\mathcal{B} \ell\right|^{2}}}{16 \pi M_{B}}\left[\left(1-\left(\frac{m_{\ell_{\alpha}}+m_{\ell_{\beta}}}{m_{B}}\right)^{2}\right)\right. \\
\left.\times\left(1-\left(\frac{m_{\ell_{\alpha}}-m_{\ell_{\beta}}}{m_{B}}\right)^{2}\right)\right]^{1 / 2} .
\end{gathered}
$$

Here

$$
\begin{aligned}
\left|\mathcal{M}_{\mathcal{B} \ell \ell}\right|^{2}= & 2\left|F_{S}\right|^{2}\left[m_{B}^{2}-\left(m_{\ell_{\alpha}}+m_{\ell_{\beta}}\right)^{2}\right] \\
& +2\left|F_{P}\right|^{2}\left[m_{B}^{2}-\left(m_{\ell_{\alpha}}-m_{\ell_{\beta}}\right)^{2}\right] \\
& +2\left|F_{V}\right|^{2}\left[m_{B}^{2}\left(m_{\ell_{\alpha}}-m_{\ell_{\beta}}\right)^{2}-\left(m_{\ell_{\alpha}}^{2}-m_{\ell_{\beta}}^{2}\right)^{2}\right] \\
& +2\left|F_{A}\right|^{2}\left[m_{B}^{2}\left(m_{\ell_{\alpha}}+m_{\ell_{\beta}}\right)^{2}-\left(m_{\ell_{\alpha}}^{2}-m_{\ell_{\beta}}^{2}\right)^{2}\right] \\
& +4 \operatorname{Re}\left(F_{S} F_{V}^{*}\right)\left(m_{\ell_{\alpha}}-m_{\ell_{\beta}}\right)\left[m_{B}^{2}+\left(m_{\ell_{\alpha}}+m_{\ell_{\beta}}\right)^{2}\right] \\
& +4 \operatorname{Re}\left(F_{P} F_{A}^{*}\right)\left(m_{\ell_{\alpha}}+m_{\ell_{\beta}}\right)\left[m_{B}^{2}-\left(m_{\ell_{\alpha}}-m_{\ell_{\beta}}\right)^{2}\right],
\end{aligned}
$$

and the $F_{X}$ coefficients are defined in terms of our Wilson coefficients as ${ }^{12}$

$F_{S}=\frac{i}{4} \frac{m_{B}^{2} f_{B}}{m_{d}+m_{d^{\prime}}}\left(E_{L L}^{S}+E_{L R}^{S}-E_{R R}^{S}-E_{R L}^{S}\right)$

$F_{P}=\frac{i}{4} \frac{m_{B}^{2} f_{B}}{m_{d}+m_{d^{\prime}}}\left(-E_{L L}^{S}+E_{L R}^{S}-E_{R R}^{S}+E_{R L}^{S}\right)$

$F_{V}=-\frac{i}{4} f_{B}\left(E_{L L}^{V}+E_{L R}^{V}-E_{R R}^{V}-E_{R L}^{V}\right)$

$F_{A}=-\frac{i}{4} f_{B}\left(-E_{L L}^{V}+E_{L R}^{V}-E_{R R}^{V}+E_{R L}^{V}\right)$,

where $f_{B} \equiv f_{B_{d, s}^{0}}$ is the $B_{d, s}^{0}$ decay constant and $m_{d, d^{\prime}}$ are the masses of the quarks contained in the $B$ meson, $B_{d}^{0} \equiv \bar{b} d$ and $B_{s}^{0} \equiv \bar{b} s$. In the lepton flavor conserving case, $\alpha=\beta$,

12 Notice that our effective Lagrangian differs from the one in [103] by a $1 /(4 \pi)^{2}$ factor. This relative factor has been absorbed in the expression for $\mathcal{M}_{\mathcal{B} \ell \ell}$, see Eq. (C.46). 


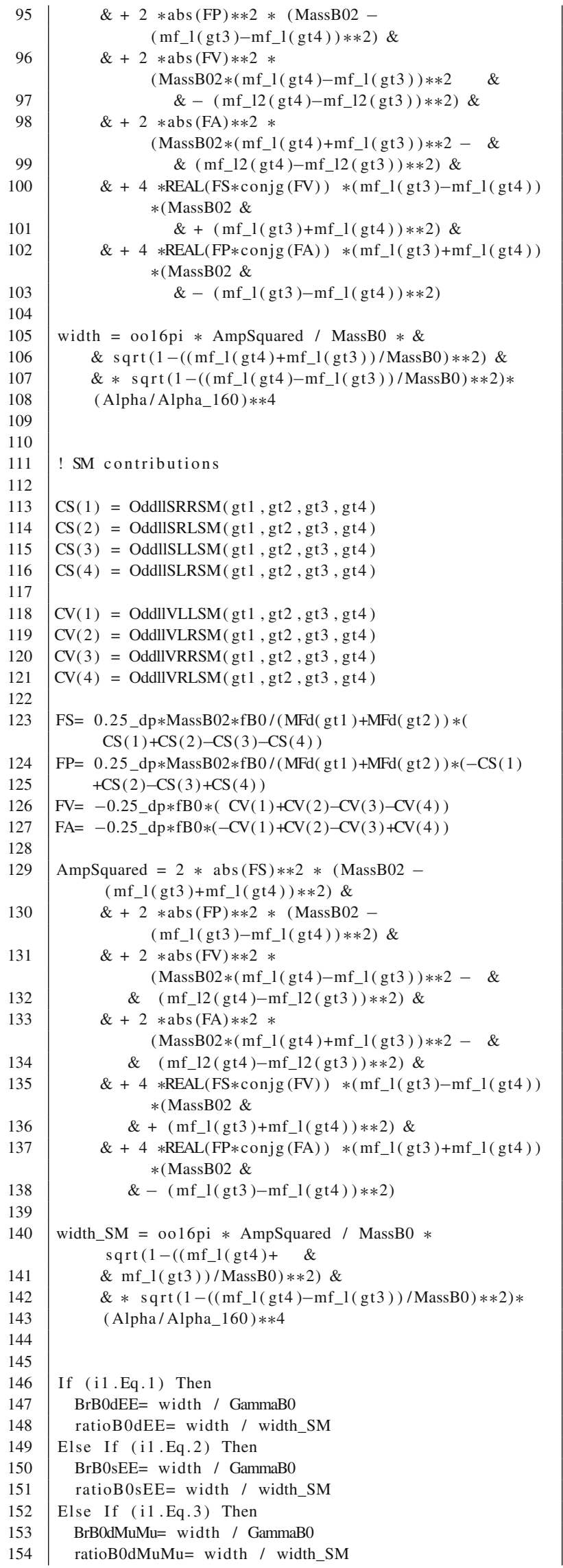

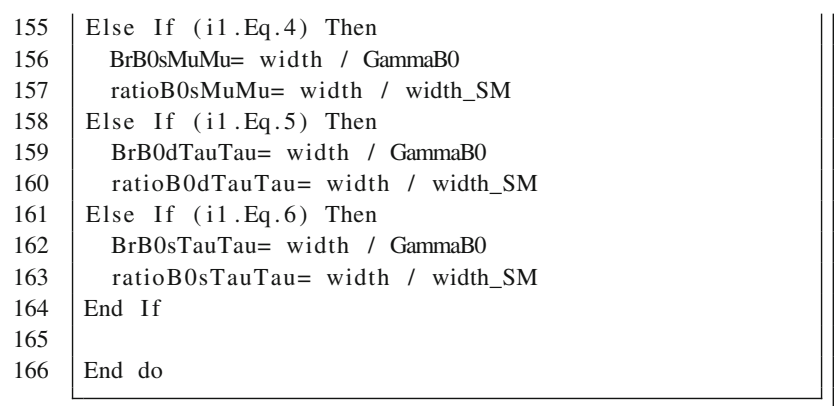

\section{C.4 $\overline{\boldsymbol{B}} \rightarrow \boldsymbol{X}_{s} \gamma$}

The branching ratio for $\bar{B} \rightarrow X_{s} \gamma$, with a cut $E_{\gamma}>1.6 \mathrm{GeV}$ in the $\bar{B}$ rest frame, can be obtained as $[104,125]$

$$
\begin{aligned}
& \mathrm{BR}\left(\bar{B} \rightarrow X_{s} \gamma\right)_{E_{\gamma}>1.6 \mathrm{GeV}} \\
& =10^{-4}\left[a_{S M}+a_{77}\left(\left|\delta C_{7}^{(0)}\right|^{2}+\left|\delta C_{7}^{\prime(0)}\right|^{2}\right)\right. \\
& +a_{88}\left(\left|\delta C_{8}^{(0)}\right|^{2}+\left|\delta C_{8}^{\prime(0)}\right|^{2}\right) \\
& +\operatorname{Re}\left(a_{7} \delta C_{7}^{(0)}+a_{8} \delta C_{8}^{(0)}\right. \\
& \left.\left.+a_{78}\left(\delta C_{7}^{(0)} \delta C_{8}^{(0) *}+\delta C_{7}^{(0)} \delta C_{8}^{\prime(0) *}\right)\right)\right],
\end{aligned}
$$

where $a_{S M}=3.15$ is the NNLO SM prediction [51,126], the other $a$ coefficients in Eq. (C.51) are found to be

$$
\begin{aligned}
a_{77} & =4.743 \\
a_{88} & =0.789 \\
a_{7} & =-7.184+0.612 i \\
a_{8} & =-2.225-0.557 i \\
a_{78} & =2.454-0.884 i
\end{aligned}
$$

and we have defined $\delta C_{i}^{(0)}=C_{i}^{(0)}-C_{i}^{(0) \mathrm{SM}}$. Finally, the $C_{i}^{(0)}$ coefficients can be written in terms of $Q_{1,2}^{L, R}$ in Eqs. (A.11) and (A.12) as

$C_{7}^{(0)}=n_{C Q} Q_{1}^{R}$

$C_{7}^{\prime(0)}=n_{C Q} Q_{1}^{L}$

$C_{8}^{(0)}=n_{C Q} Q_{2}^{R}$

$C_{8}^{\prime(0)}=n_{C Q} Q_{2}^{L}$

where $n_{C Q}^{-1}=-\frac{G_{F}}{4 \sqrt{2} \pi^{2}} V_{t b} V_{t s}^{*}$ and $V$ is the CabibboKobayashi-Maskawa (CKM) matrix. 


\section{Listing 32 bsGamma.m}

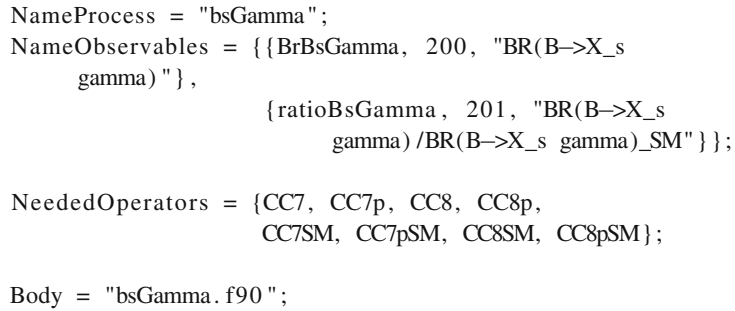

\section{Listing 33 bsGamma.f90}

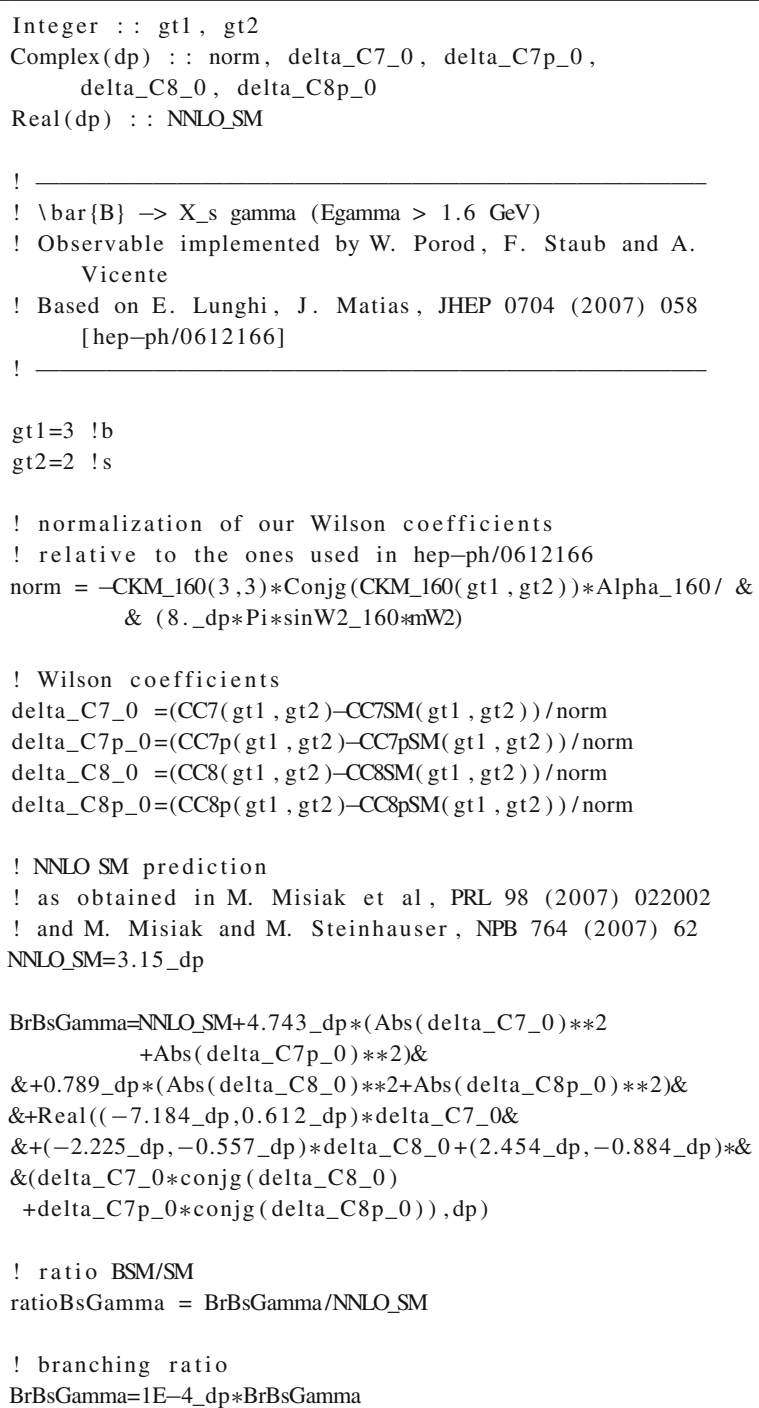

\section{C.5 $\bar{B} \rightarrow X_{s} \ell^{+} \ell^{-}$}

Our results for $\bar{B} \rightarrow X_{S} \ell^{+} \ell^{-}$are based on [106], expanded with the addition of prime operators contributions [127]. The branching ratios for the $\ell=e$ case can be written as

$$
\begin{array}{rl}
10^{7} & \mathrm{BR}\left(\bar{B} \rightarrow X_{5} e^{+} e^{-}\right)=2.3148-0.001658 \operatorname{Im}\left(R_{10}\right) \\
& +0.0005 \operatorname{Im}\left(R_{10} R_{8}^{*}+R_{10}^{\prime} R_{8}^{* *}\right) \\
& +0.0523 \operatorname{Im}\left(R_{7}\right)+0.02266 \operatorname{Im}\left(R_{7} R_{8}^{*}+R_{7}^{\prime} R_{8}^{\prime *}\right) \\
& +0.00496 \operatorname{Im}\left(R_{7} R_{9}^{*}+R_{7}^{\prime} R_{9}^{\prime *}\right) \\
& +0.00518 \operatorname{Im}\left(R_{8}\right)+0.0261 \operatorname{Im}\left(R_{8} R_{9}^{*}+R_{8}^{\prime} R_{9}^{\prime *}\right) \\
& -0.00621 \operatorname{Im}\left(R_{9}\right)-0.5420 \operatorname{Re}\left(R_{10}\right) \\
& -0.03340 \operatorname{Re}\left(R_{7}\right)+0.0153 \operatorname{Re}\left(R_{7} R_{10}^{*}+R_{7}^{\prime} R_{10}^{\prime *}\right) \\
& +0.0673 \operatorname{Re}\left(R_{7} R_{8}^{*}+R_{7}^{\prime} R_{8}^{\prime *}\right) \\
& -0.86916 \operatorname{Re}\left(R_{7} R_{9}^{*}+R_{7}^{\prime} R_{9}^{\prime *}\right)-0.0135 \operatorname{Re}\left(R_{8}\right) \\
& +0.00185 \operatorname{Re}\left(R_{8} R_{10}+R_{8}^{\prime} R_{10}^{\prime *}\right) \\
& -0.09921 \operatorname{Re}\left(R_{8} R_{9}^{*}+R_{8}^{\prime} R_{9}^{\prime *}\right)+2.833 \operatorname{Re}\left(R_{9}\right) \\
& -0.10698 \operatorname{Re}\left(R_{9} R_{10}^{*}+R_{9}^{\prime} R_{10}^{\prime *}\right) \\
& +11.0348\left(\left|R_{10}\right|^{2}+\left|R_{10}^{\prime}\right|^{2}\right) \\
& +0.2804\left(\left|R_{7}\right|^{2}+\left|R_{7}^{\prime}\right|^{2}\right) \\
& +0.003763\left(\left|R_{8}\right|^{2}+\left|R_{8}^{\prime}\right|^{2}\right) \\
& +1.527\left(\left|R_{9}\right|^{2}+\left|R_{9}^{\prime}\right|^{2}\right)
\end{array}
$$

whereas for the $\ell=\mu$ case one gets

$$
\begin{array}{rl}
10^{7} & \mathrm{BR}\left(\bar{B} \rightarrow X_{s} \mu^{+} \mu^{-}\right)=2.1774-0.001658 \operatorname{Im}\left(R_{10}\right) \\
& +0.0005 \operatorname{Im}\left(R_{10} R_{8}^{*}+R_{10}^{\prime} R_{8}^{\prime *}\right) \\
& +0.0534 \operatorname{Im}\left(R_{7}\right)+0.02266 \operatorname{Im}\left(R_{7} R_{8}^{*}+R_{7}^{\prime} R_{8}^{\prime *}\right) \\
& +0.00496 \operatorname{Im}\left(R_{7} R_{9}^{*}+R_{7}^{\prime} R_{9}^{\prime *}\right) \\
& +0.00527 \operatorname{Im}\left(R_{8}\right)+0.0261 \operatorname{Im}\left(R_{8} R_{9}^{*}+R_{8}^{\prime} R_{9}^{\prime *}\right) \\
& -0.0115 \operatorname{Im}\left(R_{9}\right)-0.5420 \operatorname{Re}\left(R_{10}\right) \\
& +0.0208 \operatorname{Re}\left(R_{7}\right)+0.0153 \operatorname{Re}\left(R_{7} R_{10}^{*}+R_{7}^{\prime} R_{10}^{\prime *}\right) \\
& +0.0648 \operatorname{Re}\left(R_{7} R_{8}^{*}+R_{7}^{\prime} R_{8}^{\prime *}\right) \\
& -0.8545 \operatorname{Re}\left(R_{7} R_{9}^{*}+R_{7}^{\prime} R_{9}^{\prime *}\right)-0.00938 \operatorname{Re}\left(R_{8}\right) \\
& +0.00185 \operatorname{Re}\left(R_{8} R_{10}+R_{8}^{\prime} R_{10}^{\prime *}\right) \\
& -0.0981 \operatorname{Re}\left(R_{8} R_{9}^{*}+R_{8}^{\prime} R_{9}^{\prime *}\right)+2.6917 \operatorname{Re}\left(R_{9}\right) \\
& -0.10698 \operatorname{Re}\left(R_{9} R_{10}^{*}+R_{9}^{\prime} R_{10}^{\prime *}\right) \\
& +10.7652\left(\left|R_{10}\right|^{2}+\left|R_{10}^{\prime}\right|^{2}\right) \\
& +0.2880\left(\left|R_{7}\right|^{2}+\left|R_{7}^{\prime}\right|^{2}\right) \\
& +0.003763\left(\left|R_{8}\right|^{2}+\left|R_{8}^{\prime}\right|^{2}\right) \\
& +1.527\left(\left|R_{9}\right|^{2}+\left|R_{9}^{\prime}\right|^{2}\right) .
\end{array}
$$

Here we have defined the ratios of Wilson coefficients

$R_{7,8}=\frac{Q_{1,2}^{R}}{Q_{1,2}^{R, \mathrm{SM}}}, \quad R_{7,8}^{\prime}=\frac{Q_{1,2}^{L}}{Q_{1,2}^{L, \mathrm{SM}}}$ 
as well as

$R_{9,10}=\frac{E_{L L}^{V} \pm E_{L R}^{V}}{E_{L L}^{V, \mathrm{SM}} \pm E_{L R}^{V, \mathrm{SM}}}, \quad R_{9,10}^{\prime}=\frac{E_{R R}^{V} \pm E_{R L}^{V}}{E_{R R}^{V, \mathrm{SM}} \pm E_{R L}^{V, \mathrm{SM}}}$

(C.60)

\section{Listing 34 BtoSLL.m}

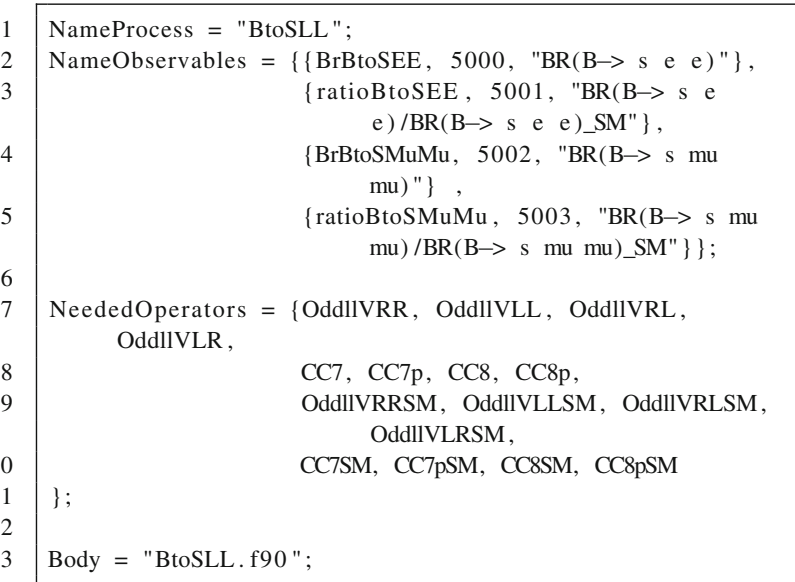

\section{Listing 35 BtoSLL.f90}

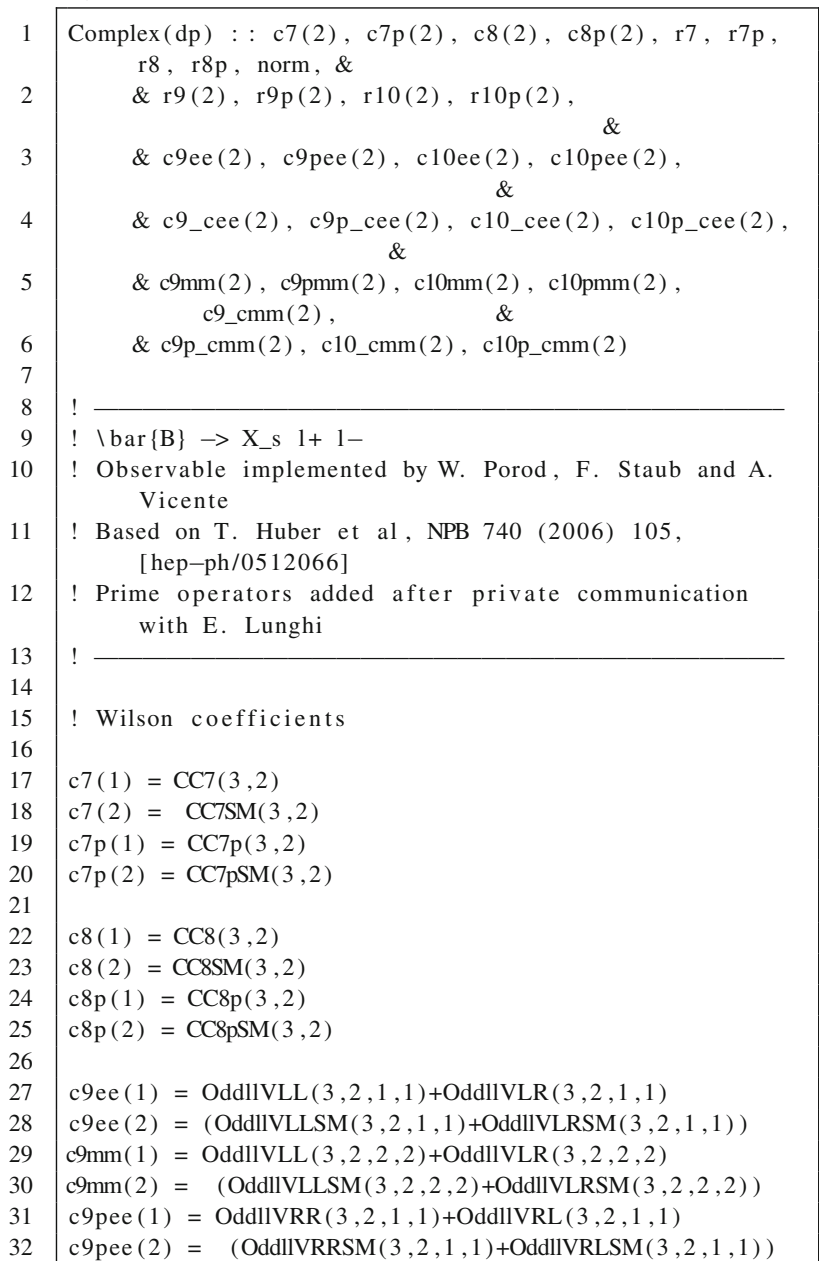

$33 \mid \mathrm{c} 9 \mathrm{pmm}(1)=\operatorname{OddllVRR}(3,2,2,2)+\operatorname{OddllVRL}(3,2,2,2)$

$34 \mathrm{c9pmm}(2)=(\operatorname{OddllVRRSM}(3,2,2,2)+\operatorname{OddllVRLSM}(3,2,2,2))$

$36 \mathrm{c} 10 \mathrm{ee}(1)=\operatorname{OddllVLL}(3,2,1,1)-\operatorname{OddllVLR}(3,2,1,1)$

$37 \mathrm{c} 10 \mathrm{ee}(2)=(\operatorname{OddlIVLLSM}(3,2,1,1)-\operatorname{OddlIVLRSM}(3,2,1,1))$

$38 \mathrm{cl} 10 \mathrm{~mm}(1)=\operatorname{OddllVLL}(3,2,2,2)-\operatorname{OddllIVLR}(3,2,2,2)$

$39 \operatorname{cl} 10 \mathrm{~mm}(2)=(\operatorname{OddllVLLSM}(3,2,2,2)-\operatorname{OddllVLRSM}(3,2,2,2))$

$40 \mathrm{c}$ 10pee $(1)=\operatorname{OddllVRR}(3,2,1,1)-\operatorname{Odd} \operatorname{llVRL}(3,2,1,1)$

41 c10pee $(2)=(\operatorname{OddllVRRSM}(3,2,1,1)-\operatorname{OddllVRLSM}(3,2,1,1))$

$42 \operatorname{c10pmm}(1)=\operatorname{OddllVRR}(3,2,2,2)-\operatorname{OddllVRL}(3,2,2,2)$

$43 \operatorname{cl0pmm}(2)=(\operatorname{OddllVRRSM}(3,2,2,2)-\operatorname{OddllVRLSM}(3,2,2,2))$

45 ! ratios

46

47

48

49

50

51

52

$54 \mathrm{r} 9 \mathrm{p}(1)=\mathrm{c} 9 \mathrm{pee}(1) / \mathrm{c} 9 \mathrm{ee}(2)$

$55 \mathrm{r} 9 \mathrm{p}(2)=\mathrm{c} 9 \mathrm{pmm}(1) / \mathrm{c} 9 \mathrm{~mm}(2)$

$57 \mathrm{r} 10(1)=\mathrm{c} 10 \mathrm{ee}(1) / \mathrm{c} 10 \mathrm{ee}(2)$

$58 \mathrm{r} 10(2)=\mathrm{c} 10 \mathrm{~mm}(1) / \mathrm{c} 10 \mathrm{~mm}(2)$

$59 \mathrm{r} 10 \mathrm{p}(1)=\mathrm{c} 10 \mathrm{pee}(1) / \mathrm{c} 10 \mathrm{ee}(2)$

$60 \mathrm{r} 10 \mathrm{p}(2)=\mathrm{c} 10 \mathrm{pmm}(1) / \mathrm{c} 10 \mathrm{~mm}(2)$

61

62

$\operatorname{BrBtoSEE}=\left(2.3148 \_\mathrm{dp}-1.658 \mathrm{e}-3 \_\mathrm{dp} * \operatorname{Aimag}(\mathrm{R} 10(1))\right.$ \&

$\&+5 \cdot \mathrm{e}-4 \_\mathrm{dp} * \operatorname{Aimag}(\mathrm{r} 10(1) * \operatorname{Conjg}(\mathrm{r} 8)+$ $\mathrm{r} 10 \mathrm{p}(1) * \operatorname{Conjg}(\mathrm{r} 8 \mathrm{p}))$

$\mathrm{r} 8)$
$\&$

$\&+5.23 \mathrm{e}-2 \_\mathrm{dp} * \operatorname{Aimag}(\mathrm{r} 7)+5.18 \mathrm{e}-3 \_\mathrm{dp} *$ Aimag $(\mathrm{r} 8)$

$\&+2.266 \mathrm{e}-2 \_\mathrm{dp} * \operatorname{Aimag}(\mathrm{r} 7 * \operatorname{Conjg}(\mathrm{r} 8)+\mathrm{r} 7 \mathrm{p} *$ Conjg (r8p) ) \&

66

$\&+4.96 \mathrm{e}-3 \_\mathrm{dp} * \operatorname{Aimag}(\mathrm{r} 7 * \operatorname{Conjg}(\mathrm{r} 9(1))+\mathrm{r} 7 \mathrm{p} *$ Conjg(r9p (1)) ) \&

67

$\&+2.61 \mathrm{e}-2 \_\mathrm{dp} * \operatorname{Aimag}(\mathrm{r} 8 * \operatorname{Conjg}(\mathrm{r} 9(1))+\mathrm{r} 8 \mathrm{p} *$ Conjg(r9p (1)) \&

68

$\&-6.21 \mathrm{e}-3 \_\mathrm{dp} * \operatorname{Aimag}(\mathrm{r} 9(1))-0.5420 \_\mathrm{dp} * \operatorname{Real}($ $\mathrm{r} 10(1), \mathrm{dp}) \quad \&$

69

$\&-3.340 \mathrm{e}-2 \_\mathrm{dp} * \operatorname{Real}(\mathrm{r} 7, \mathrm{dp})-1.35 \mathrm{e}-2 \_\mathrm{dp} *$ $\operatorname{Real}(\mathrm{r} 8, \mathrm{dp})$ $\&$

70

71

72

73

73

74

75

76

77

78

78
79

79

80

81

82

83
84

85

86

87

$\&+1.53 \mathrm{e}-2 \_\mathrm{dp} * \operatorname{Real}(\mathrm{r} 7 * \operatorname{Conjg}(\mathrm{r} 10(1))+$ $\mathrm{r} 7 \mathrm{p} * \operatorname{Conjg}(\mathrm{r} 10 \mathrm{p}(1)), \mathrm{dp}) \quad \&$

$\&+6.73 \mathrm{e}-2 \_\mathrm{dp} * \operatorname{Real}(\mathrm{r} 7 * \operatorname{Conjg}(\mathrm{r} 8)+\mathrm{r} 7 \mathrm{p} *$ Conjg (r8p), dp ) \&

$\&-0.86916$ dp $*$ Real $(\mathrm{r} 7 * \operatorname{Conjg}(\mathrm{r} 9(1))+$ $\mathrm{r} 7 \mathrm{p} *$ Conjg $(\mathrm{r} 9 \mathrm{p}(1)), \mathrm{dp}) \quad \&$

$\&+1.85 \mathrm{e}-3 \_\mathrm{dp} * \operatorname{Real}(\mathrm{r} 8 * \operatorname{Conjg}(\mathrm{r} 10(1))+$ $\mathrm{r} 8 \mathrm{p} * \operatorname{Conjg}(\mathrm{r} 10 \mathrm{p}(1)), \mathrm{dp}) \quad \&$

$\&-9.921 \mathrm{e}-2 \_\mathrm{dp} * \operatorname{Real}(\mathrm{r} 8 * \operatorname{Conjg}(\mathrm{r} 9(1))+$ $\mathrm{r} 8 \mathrm{p} * \operatorname{Conjg}(\mathrm{r} 9 \mathrm{p}(1)), \mathrm{dp}) \quad \&$

$\&+2.833 \_d p * \operatorname{Real}(\mathrm{r} 9(1), \mathrm{dp})+0.2804 \_\mathrm{dp} *$ $(\operatorname{Abs}(\mathrm{r} 7) * * 2+\operatorname{Abs}(\mathrm{r} 7 \mathrm{p}) * * 2) \&$

$\&-0.10698 \_d p * \operatorname{Real}($ r9(1) $*$ Conjg $(\mathrm{r} 10(1))$

\&

$+\mathrm{r} 9 \mathrm{p}(1) *$ Conjg $(\mathrm{r} 10 \mathrm{p}(1)), \mathrm{dp})$ $\&$

$\&+11.0348 \_\mathrm{dp} *(\operatorname{Abs}(\mathrm{r} 10(1)) * * 2+\operatorname{Abs}(\mathrm{r} 10 \mathrm{p}(1)) * * 2)$ \&

$\&+1.527 \_\mathrm{dp} *(\operatorname{Abs}(\mathrm{r} 9(1)) * * 2+\operatorname{Abs}(\mathrm{r} 9 \mathrm{p}(1)) * * 2)$ \&

$\left.\&+3.763 \mathrm{e}-3 \_\mathrm{dp} *(\operatorname{Abs}(\mathrm{r} 8) * * 2+\operatorname{Abs}(\mathrm{r} 8 \mathrm{p}) * * 2)\right)$

! ratio $\mathrm{BR}(\mathrm{B} \rightarrow \mathrm{Xs}$ mu+ $\mathrm{mu}-) / \mathrm{BR}(\mathrm{B} \rightarrow \mathrm{Xs}$ e+ e-) $\mathrm{SM}$

ratioBtoSee $=$ BrBtoSEE/16.5529_dp

! branching ratio $\mathrm{B} \rightarrow \mathrm{Xs}$ e+ e-

BrBtoSEE $=$ BrBtoSEE $*$ 1.e-7_dp 


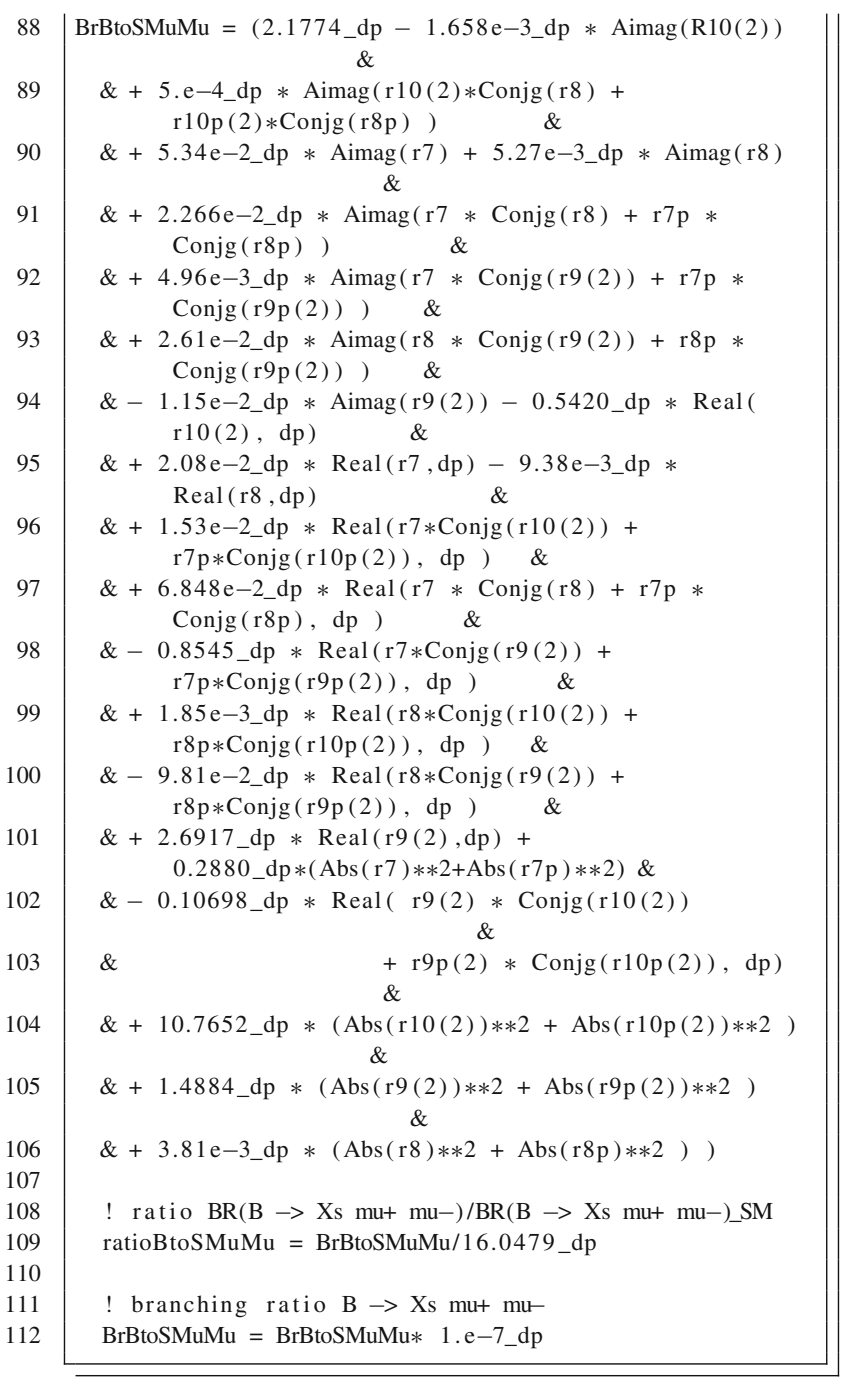

\section{C.6 $\mathrm{B}^{+} \rightarrow \mathrm{K}^{+} \ell^{+} \ell^{-}$}

Our results for $B^{+} \rightarrow K^{+} \ell^{+} \ell^{-}$are based on the expressions given in [102]. The branching ratio for $B^{+} \rightarrow K^{+} \mu^{+} \mu^{-}$ in the high- $q^{2}$ region, $q^{2}$ being the dilepton invariant mass squared, can be written as

$$
\begin{aligned}
& \mathrm{BR}\left(B^{+} \rightarrow K^{+} \mu^{+} \mu^{-}\right)_{q^{2} \in[14.18,22] \mathrm{GeV}^{2}} \simeq 1.11 \\
& +0.22\left(C_{7}^{\mathrm{NP}}+C_{7}^{\prime}\right)+0.27\left(C_{9}^{\mathrm{NP}}+C_{9}^{\prime}\right) \\
& -0.27\left(C_{10}^{\mathrm{NP}}+C_{10}^{\prime}\right) .
\end{aligned}
$$

The coefficients in Eq. (C.61) can be related to the ones in our generic Lagrangian as

$$
C_{7}^{\mathrm{NP}}=n_{C Q}\left(Q_{1}^{R}-Q_{1}^{R, \mathrm{SM}}\right)
$$

$C_{7}^{\prime}=n_{C Q} Q_{1}^{L}$

$$
\begin{aligned}
& C_{9}^{\mathrm{NP}}=n_{C Q}\left[\left(E_{L L}^{V}+E_{L R}^{V}\right)-\left(E_{L L}^{V, \mathrm{SM}}+E_{L R}^{V, \mathrm{SM}}\right)\right] \\
& C_{9}^{\prime}=n_{C Q}\left(E_{R R}^{V}+E_{R L}^{V}\right) \\
& C_{10}^{\mathrm{NP}}=n_{C Q}\left[\left(E_{L L}^{V}-E_{L R}^{V}\right)-\left(E_{L L}^{V, \mathrm{SM}}-E_{L R}^{V, \mathrm{SM}}\right)\right] \\
& C_{10}^{\prime}=n_{C Q}\left(E_{R R}^{V}-E_{R L}^{V}\right)
\end{aligned}
$$

where the normalization factor $n_{C Q}$ was already defined after Eq. (C.56).

\section{Listing 36 BtoKLL.m}

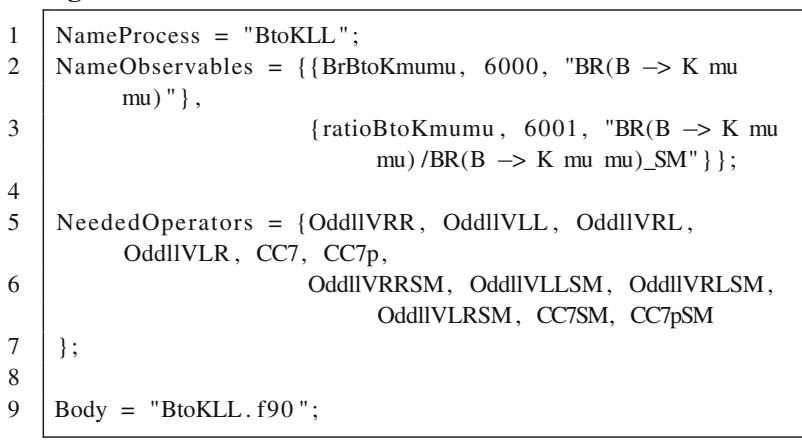

\section{Listing 37 BtoKLL.f90}

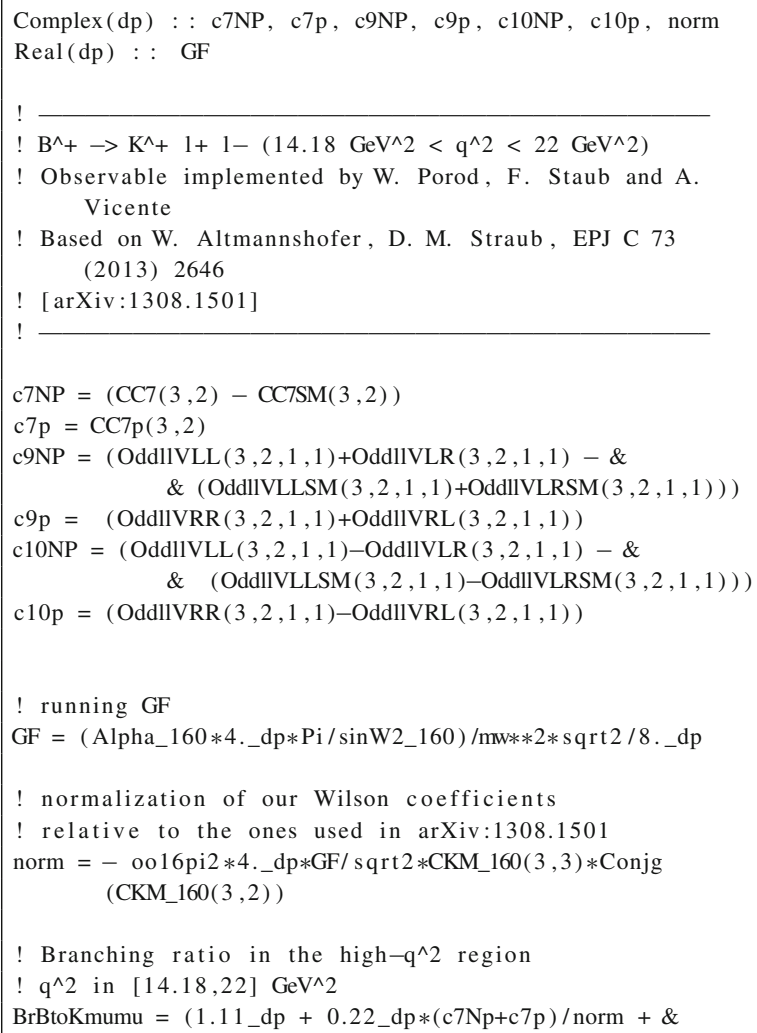




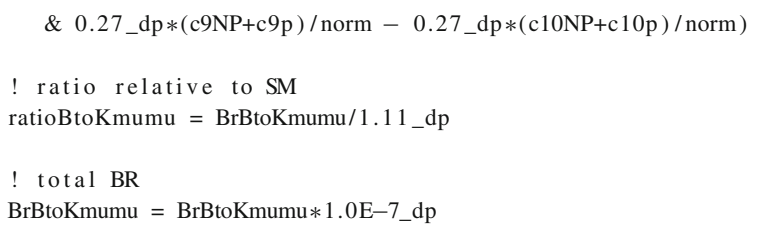

\section{C.7 $\bar{B} \rightarrow X_{d, s} v \bar{v}$}

The branching ratio for $\bar{B} \rightarrow X_{q} v \bar{v}$, with $q=d$, s, is given by [105]

$$
\begin{aligned}
\operatorname{BR}\left(\bar{B} \rightarrow X_{q} \nu \bar{v}\right) \\
=\frac{\alpha^{2}}{4 \pi^{2} \sin ^{4} \theta_{W}} \frac{\left|V_{t b} V_{t q}^{*}\right|^{2}}{\left|V_{c b}\right|^{2}} \frac{\operatorname{BR}\left(\bar{B} \rightarrow X_{c} e \bar{v}_{e}\right) \kappa(0)}{f\left(\hat{m}_{c}\right) \kappa\left(\hat{m}_{c}\right)} \\
\quad \times \sum_{f}\left[\left(\left|c_{L}\right|^{2}+\left|c_{R}\right|^{2}\right) f\left(\hat{m}_{q}\right)-4 \operatorname{Re}\left(c_{L} c_{R}^{*}\right) \hat{m}_{q} \tilde{f}\left(\hat{m}_{q}\right)\right] .
\end{aligned}
$$

The sum runs over the three neutrinos and $\hat{m}_{i} \equiv m_{i} / m_{b}$. The functions $f\left(\hat{m}_{c}\right)$ and $\kappa\left(\hat{m}_{c}\right)$ represent the phase-space and the 1-loop QCD corrections, respectively. In case of $\kappa\left(\hat{m}_{c}\right)$, one needs the numerical values $\kappa(0)=0.83$ and $\kappa\left(\hat{m}_{c}\right)=0.88$. The functions $f(x)$ and $\tilde{f}(x)$ take the form

$f(x)=1-8 x^{2}+8 x^{6}-x^{8}-24 x^{4} \log x$

$\tilde{f}(x)=1+9 x^{2}-9 x^{4}-x^{6}+12 x^{2}\left(1+x^{2}\right) \log x$.

Finally, BR $\left(\bar{B} \rightarrow X_{c} e \bar{v}_{e}\right)_{\exp }=0.101$ [128] and the coefficients $c_{L}$ and $c_{R}$ are given by

$c_{L}=n_{B X v v}^{q} F_{L L}^{V}$

$c_{R}=n_{B X v v}^{q} F_{R L}^{V}$,

where $\left(n_{B X v v}^{q}\right)^{-1}=\frac{4 G_{F}}{\sqrt{2}} \frac{\alpha}{2 \pi \sin ^{2} \theta_{W}} V_{t b}^{*} V_{t q}$ is the relative factor between our Wilson coefficients and the ones in [105].

\section{Listing 38 BtoQnunu.m}

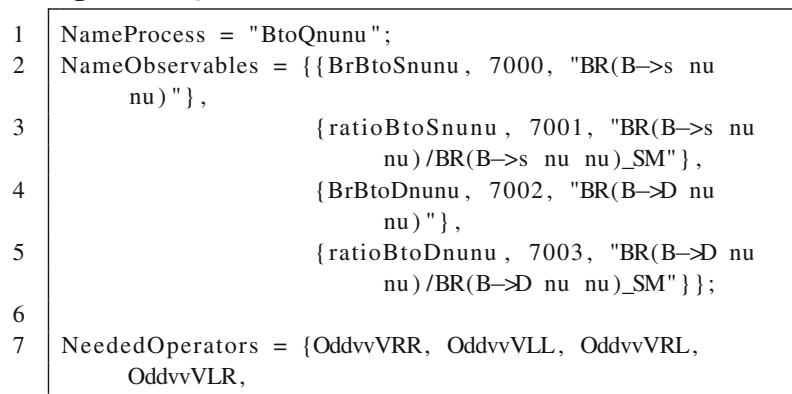

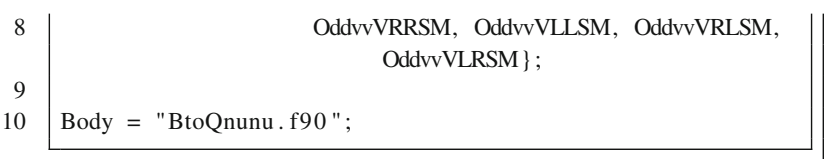

\section{Listing 39 BtoQnunu.f90}

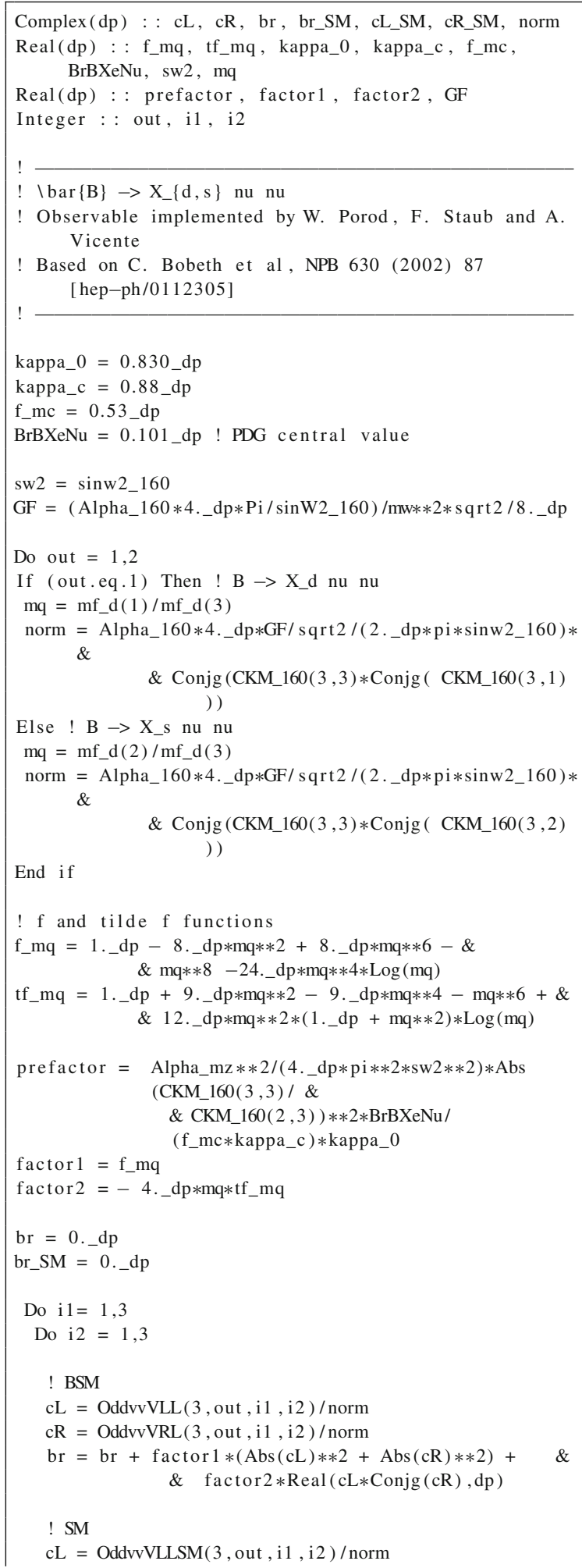


$\mathrm{cR}=\operatorname{OddvvVRLSM}(3$, out $, \mathrm{i} 1, \mathrm{i} 2) /$ norm

br_SM $=$ br_SM + factor $1 *(\operatorname{Abs}(\mathrm{cL}) * * 2+\operatorname{Abs}(\mathrm{cR}) * * 2)+$ $\&$

$$
\& \text { factor } 2 * \operatorname{Real}(\mathrm{cL} * \operatorname{Conjg}(\mathrm{cR}), \mathrm{dp})
$$

End Do

End do

If (out.eq.1) Then ! B $\rightarrow$ X d nu nu

BrBtoDnunu $=$ prefactor $*$ br $* \operatorname{Abs}\left(C K M \_160(3,1)\right) * * 2$

ratioBtoDnunu $=$ br/br SM

Else ! B $\rightarrow$ X_s nu nu

BrBtoSnunu $=$ prefactor $*$ br $*$ Abs $($ CKM_160 $(3,2)) * * 2$

ratioBtoSnunu $=$ br/br_SM

End if

End Do

\section{$\mathrm{C} .8 K \rightarrow \pi v \bar{v}$}

Following [105], the branching ratios for rare Kaon decays involving neutrinos in the final state can be written as

$$
\begin{aligned}
& \operatorname{BR}\left(K^{+} \rightarrow \pi^{+} \nu \bar{v}\right)=2 r_{1} r_{2} r_{K^{+}} \sum_{f}\left[\left(\operatorname{Im} \lambda_{t} X_{f}\right)^{2}\right. \\
& \left.\quad+\left(\operatorname{Re} \lambda_{c} X_{N L}+\operatorname{Re} \lambda_{t} X_{f}\right)^{2}\right]
\end{aligned}
$$

$\operatorname{BR}\left(K_{L} \rightarrow \pi^{0} v \bar{v}\right)=2 r_{1} r_{K_{L}} \sum_{f}\left(\operatorname{Im} \lambda_{t} X_{f}\right)^{2}$

where the sums are over the three neutrino species, $X_{N L}=$ 9.78 $\cdot 10^{-4}$ is the SM NLO charm correction [48,129], $\lambda_{t}=$ $V_{t s}^{*} V_{t d}$ and $\lambda_{c}=V_{c s}^{*} V_{c d}$, the coefficients $r_{1}, r_{2}, r_{K^{+}}$and $r_{K_{L}}$ take the numerical values

$r_{1}=1.17 \cdot 10^{-4}$

$r_{2}=0.24$

$r_{K^{+}}=0.901$

$r_{K_{L}}=0.944$

and $X_{f}$ contains the Wilson coefficients contributing to the processes, $F_{L L}^{V}$ and $F_{R L}^{V}$, as

$X_{f}=n_{K \pi \nu v}\left(F_{L L}^{V}+F_{R L}^{V}\right)$.

Here $n_{K \pi \nu v}^{-1}=\frac{4 G_{F}}{\sqrt{2}} \frac{\alpha}{2 \pi \sin ^{2} \theta_{W}} V_{t s}^{*} V_{t d}$

\section{Listing 40 KtoPInunu.m}

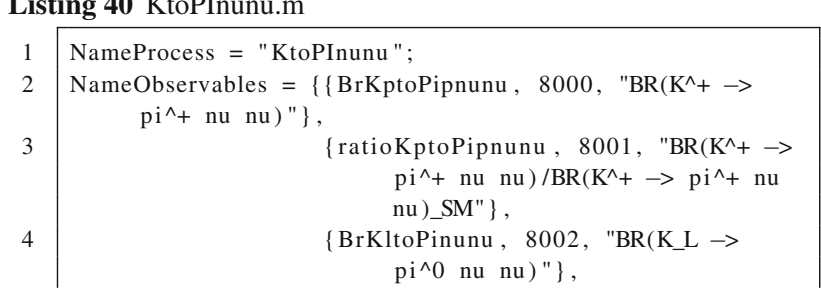

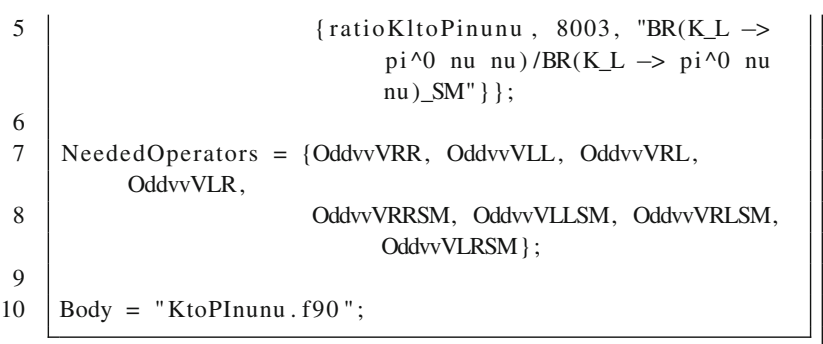

\section{Listing 41 KtoPInunu.f90}

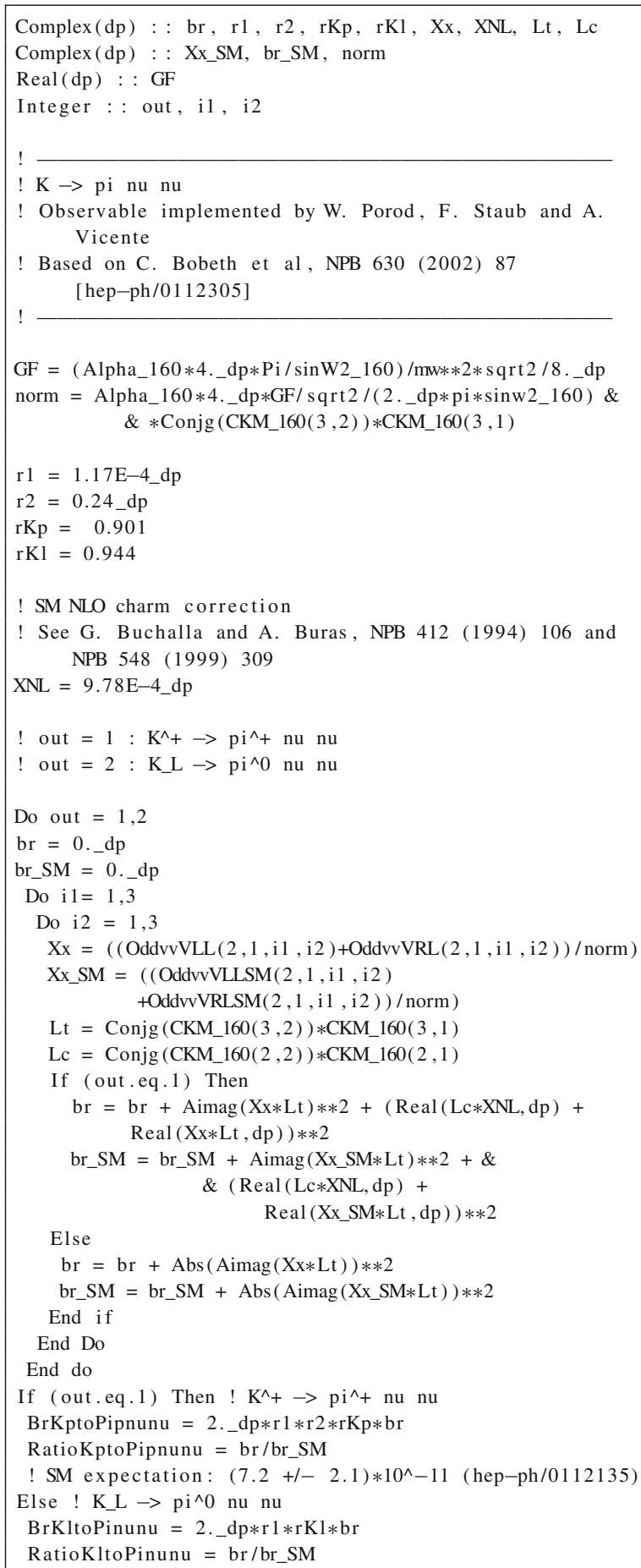




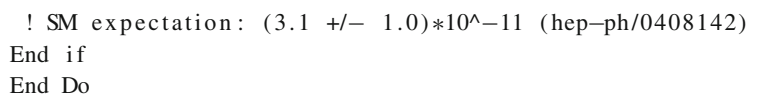

\section{C.9 $\Delta \boldsymbol{M}_{\boldsymbol{B}_{s, d}}$}

The $B_{q}^{0}-\bar{B}_{q}^{0}$ mass difference can be written as $[108,130]$

$\Delta M_{B_{q}}=\frac{G_{F}^{2} m_{W}^{2}}{6 \pi^{2}} m_{B_{q}} \eta_{B} f_{B_{q}}^{2} \hat{B}_{B_{q}}\left|V_{t q}^{\text {eff }}\right|^{2}\left|F_{t t}^{q}\right|$,

where $q=s, d, m_{B_{q}}$ and $f_{B_{q}}$ are the $B_{q}^{0}$ mass and decay constant, respectively, $\eta_{B}=0.55$ is a QCD factor [47,131], $\hat{B}_{B_{q}}$ is a non-perturbative parameter (with values $\hat{B}_{B_{d}}=1.26$ and $\hat{B}_{B_{s}}=1.33$, obtained from recent lattice computations [132]) and $\left|V_{t q}^{\text {eff }}\right|^{2}=\left(V_{t b}^{*} V_{t q}\right)^{2} . F_{t t}^{q}$ is given by

$$
\begin{aligned}
F_{t t}^{q} & =S_{0}\left(x_{t}\right)+\frac{1}{4 r} C_{\text {new }}^{V L L} \\
& +\frac{1}{4 r} C_{1}^{V R R}+\bar{P}_{1}^{L R} C_{1}^{L R}+\bar{P}_{2}^{L R} C_{2}^{L R} \\
& +\bar{P}_{1}^{S L L}\left(C_{1}^{S L L}+C_{1}^{S R R}\right)+\bar{P}_{2}^{S L L}\left(C_{2}^{S L L}+C_{2}^{S R R}\right)
\end{aligned}
$$

where $r=0.985$ [47], $x_{t}=\frac{m_{t}^{2}}{m_{W}^{2}}$, with $m_{t}$ the top quark mass, the $\bar{P}$ coefficients take the numerical values

$$
\begin{aligned}
\bar{P}_{1}^{L R} & =-0.71 \\
\bar{P}_{2}^{L R} & =0.90 \\
\bar{P}_{1}^{S L L} & =-0.37 \\
\bar{P}_{2}^{S L L} & =-0.72
\end{aligned}
$$

and the function

$S_{0}\left(x_{t}\right)=\frac{4 x_{t}-11 x_{t}^{2}+x_{t}^{3}}{4\left(1-x_{t}\right)^{2}}-\frac{3 x_{t}^{3} \log x_{t}}{2\left(1-x_{t}\right)^{3}}$

was introduced by Inami and Lim in [133] and given, for example, in [134]. Finally, the coefficients in Eq. (C.78) are related to the $D_{X Y}^{I}$ coefficients in Eq. (A.13) as

$$
\begin{aligned}
& C_{\text {new }}^{V L L}=n_{\Delta}^{q}\left(D_{L L}^{V}-D_{L L}^{V, \mathrm{SM}}\right) \\
& C_{1}^{V R R}=n_{\Delta}^{q} D_{R R}^{V} \\
& C_{1}^{L R}=n_{\Delta}^{q}\left(D_{L R}^{V}+D_{R L}^{V}\right) \\
& C_{2}^{L R}=n_{\Delta}^{q}\left(D_{L R}^{S}+D_{R L}^{S}+\delta_{2}^{L R}\right)
\end{aligned}
$$

$C_{1}^{S L L}=n_{\Delta}^{q}\left(D_{L L}^{S}+\delta_{1}^{S L L}\right)$

$C_{1}^{S R R}=n_{\Delta}^{q}\left(D_{R R}^{S}+\delta_{1}^{S R R}\right)$

$C_{2}^{S L L}=n_{\Delta}^{q} D_{L L}^{T}$

$C_{2}^{S R R}=n_{\Delta}^{q} D_{R R}^{T}$

where the factor $\left(n_{\Delta}^{q}\right)^{-1}=\frac{G_{F}^{2} m_{W}^{2}}{16 \pi^{2}}\left|V_{t q}^{\text {eff }}\right|^{2}$ normalizes our Wilson coefficients to the ones in $[108,130]$. The corrections $\delta_{2}^{L R}, \delta_{1}^{S L L}$ and $\delta_{1}^{S R R}$ are induced by double penguin diagrams mediated by scalar and pseudoscalar states $[108,130]$. These 2-loop contributions may have a sizable impact in some models, and their inclusion is necessary in order to achieve a precise result for $\Delta M_{B_{q}}$. They can be written as

$\delta_{2}^{L R}=-\frac{H_{L}^{S, P}\left(H_{R}^{S, P}\right)^{*}}{m_{S, P}^{2}}$

\begin{tabular}{|c|}
\hline 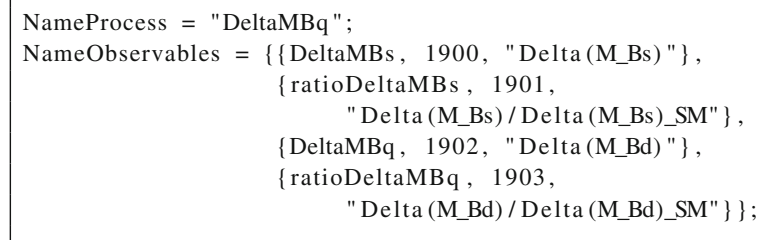 \\
\hline $\begin{aligned} \text { Externalstates }= & \{\mathrm{Fd}\} ; \\
\text { NeededOperators }= & \text { OO4dSLL, O4dSRR, O4dSRL, O4dSLR, } \\
\text { O4dVRR, O4dVLL, } & \text { O4dVLLSM, O4dVRL, O4dVLR, O4dTLL, } \\
& \text { O4dTLR, O4dTRL, O4dTRR }\} ;\end{aligned}$ \\
\hline $\begin{array}{l}\text { IncludeSMprediction ["DeltaMBq"] = False; } \\
\text { Body = "DeltaMBq.f90"; }\end{array}$ \\
\hline
\end{tabular}

$\delta_{1}^{S L L}=-\frac{\left(H_{L}^{S, P}\right)^{2}}{2 m_{S, P}^{2}}$

$\delta_{1}^{S R R}=-\frac{\left(H_{L}^{S, P}\right)^{2}}{2 m_{S, P}^{2}}$

where $H_{L}^{S, P}$ and $H_{R}^{S, P}$ are defined in Eq. (A.17). The double penguin corrections in Eqs. (C.89)-(C.91) are obtained by summing up over all scalar and pseudoscalar states in the model.

Listing 42 DeltaMBq.m

\section{Listing 43 DeltaMBq.f90}

\begin{tabular}{c|c||}
\cline { 2 - 3 } 1 & Complex (dp) : : MBq, etaB, FBq2, BBq, Ftt, Veff2, r, \& \\
2 & $\&$ P1bLR, P2bLR, P1bSLL, P2bSLL, norm, \&
\end{tabular} 

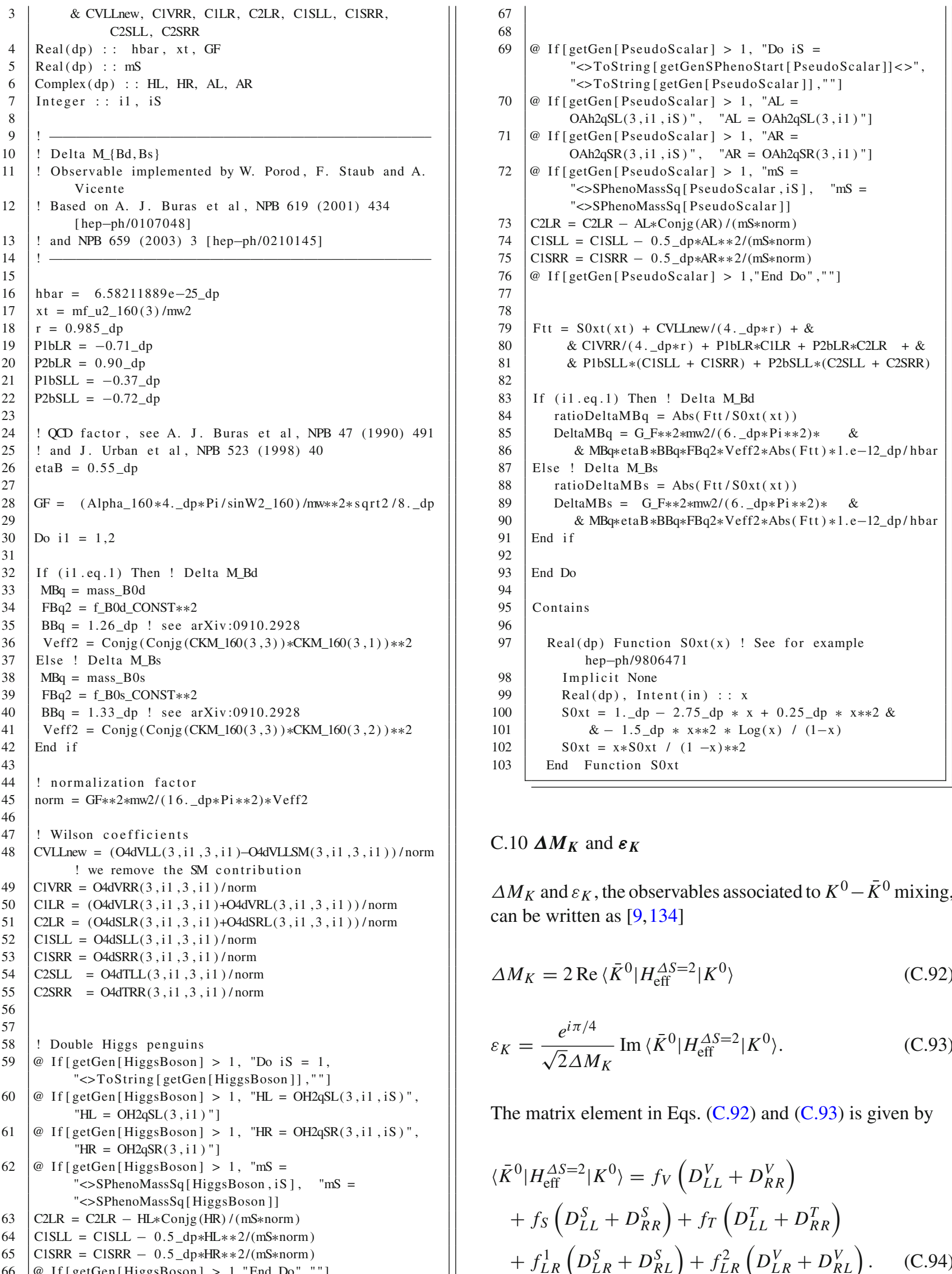

\section{C.10 $\Delta M_{K}$ and $\varepsilon_{K}$}

$\Delta M_{K}$ and $\varepsilon_{K}$, the observables associated to $K^{0}-\bar{K}^{0}$ mixing, can be written as $[9,134]$

$$
\begin{aligned}
& \Delta M_{K}=2 \operatorname{Re}\left\langle\bar{K}^{0}\left|H_{\mathrm{eff}}^{\Delta S=2}\right| K^{0}\right\rangle \\
& \varepsilon_{K}=\frac{e^{i \pi / 4}}{\sqrt{2} \Delta M_{K}} \operatorname{Im}\left\langle\bar{K}^{0}\left|H_{\mathrm{eff}}^{\Delta S=2}\right| K^{0}\right\rangle .
\end{aligned}
$$

The matrix element in Eqs. (C.92) and (C.93) is given by

$$
\begin{aligned}
& \left\langle\bar{K}^{0}\left|H_{\mathrm{eff}}^{\Delta S=2}\right| K^{0}\right\rangle=f_{V}\left(D_{L L}^{V}+D_{R R}^{V}\right) \\
& \quad+f_{S}\left(D_{L L}^{S}+D_{R R}^{S}\right)+f_{T}\left(D_{L L}^{T}+D_{R R}^{T}\right) \\
& \quad+f_{L R}^{1}\left(D_{L R}^{S}+D_{R L}^{S}\right)+f_{L R}^{2}\left(D_{L R}^{V}+D_{R L}^{V}\right)
\end{aligned}
$$


The $f$ coefficients are

$$
\begin{aligned}
& f_{V}=\frac{1}{3} m_{K} f_{K}^{2} B_{1}^{V L L}(\mu) \\
& f_{S}=-\frac{5}{24}\left(\frac{m_{K}}{m_{s}(\mu)+m_{d}(\mu)}\right)^{2} m_{K} f_{K}^{2} B_{1}^{S L L}(\mu) \\
& f_{T}=-\frac{1}{2}\left(\frac{m_{K}}{m_{s}(\mu)+m_{d}(\mu)}\right)^{2} m_{K} f_{K}^{2} B_{2}^{S L L}(\mu) \\
& f_{L R}^{1}=-\frac{1}{6}\left(\frac{m_{K}}{m_{S}(\mu)+m_{d}(\mu)}\right)^{2} m_{K} f_{K}^{2} B_{1}^{L R}(\mu) \\
& f_{L R}^{2}=\frac{1}{4}\left(\frac{m_{K}}{m_{S}(\mu)+m_{d}(\mu)}\right)^{2} m_{K} f_{K}^{2} B_{2}^{L R}(\mu)
\end{aligned}
$$

where $\mu=2 \mathrm{GeV}$ is the energy scale at which the matrix element is computed and $f_{K}$ the Kaon decay constant. The values of the quark masses at $\mu=2 \mathrm{GeV}$ are given by $m_{d}(\mu)=7 \mathrm{MeV}$ and $m_{s}(\mu)=125 \mathrm{MeV}$ (see table 1 in [98]), whereas the $B_{i}^{X}$ coefficients have the following values at $\mu=2 \mathrm{GeV}$ [135]: $B_{1}^{V L L}(\mu)=0.61, B_{1}^{S L L}(\mu)=0.76$, $B_{2}^{S L L}(\mu)=0.51, B_{1}^{L R}(\mu)=0.96$ and $B_{2}^{L R}(\mu)=1.3$.

As in [9], we treat the SM contribution separately. We define $D_{L L}^{V}=D_{L L}^{V, S M}+D_{L L}^{V, B S M}$. For $D_{L L}^{V, B S M}$ one just subtracts the SM contributions to $D_{L L}^{V}$, whereas for $D_{L L}^{V, S M}$ one can use the results in [136-138], where the relevant QCD corrections are included,

$$
\begin{aligned}
D_{L L}^{V, S M}= & \frac{G_{F}^{2} m_{W}^{2}}{4 \pi^{2}}\left[\lambda_{c}^{* 2} \eta_{1} S_{0}\left(x_{c}\right)+\lambda_{t}^{* 2} \eta_{2} S_{0}\left(x_{t}\right)\right. \\
& \left.+2 \lambda_{c}^{*} \lambda_{t}^{*} \eta_{3} S_{0}\left(x_{c}, x_{t}\right)\right] .
\end{aligned}
$$

Here $x_{i}=m_{i}^{2} / m_{w}^{2}, \lambda_{i}=V_{i s}^{*} V_{i d}$ and $S_{0}(x)$ and $S_{0}(x, y)$ are the Inami-Lim functions [133]. $S_{0}(x)$ was already defined in Eq. (C.80), whereas $S_{0}\left(x_{c}, x_{t}\right)$ is given by [134]

$$
S_{0}\left(x_{c}, x_{t}\right)=x_{c}\left[\log \frac{x_{t}}{x_{c}}-\frac{3 x_{t}}{4\left(1-x_{t}\right)}-\frac{3 x_{t}^{2} \log x_{t}}{4\left(1-x_{t}\right)^{2}}\right] \text {. }
$$

In the last expression we have kept only terms linear in $x_{c} \ll 1$. Finally, the $\eta_{i}$ coefficients comprise short distance QCD corrections. Their numerical values are $\eta_{1,2,3}=$ $(1.44,0.57,0.47)[138] .{ }^{13}$

\footnotetext{
$\overline{13}$ Note that we have chosen a value for $\eta_{1}$ which results from our numerical values for $\alpha_{s}\left(m_{Z}\right)$ and $m_{c}\left(m_{c}\right)$, see table 5 in [138].
}

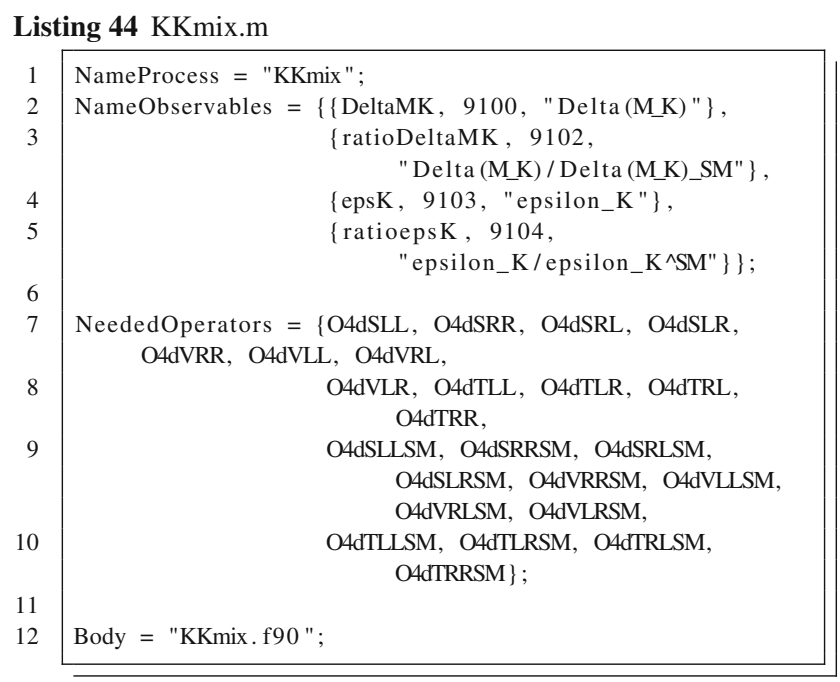

\section{Listing 45 KKmix.f90}

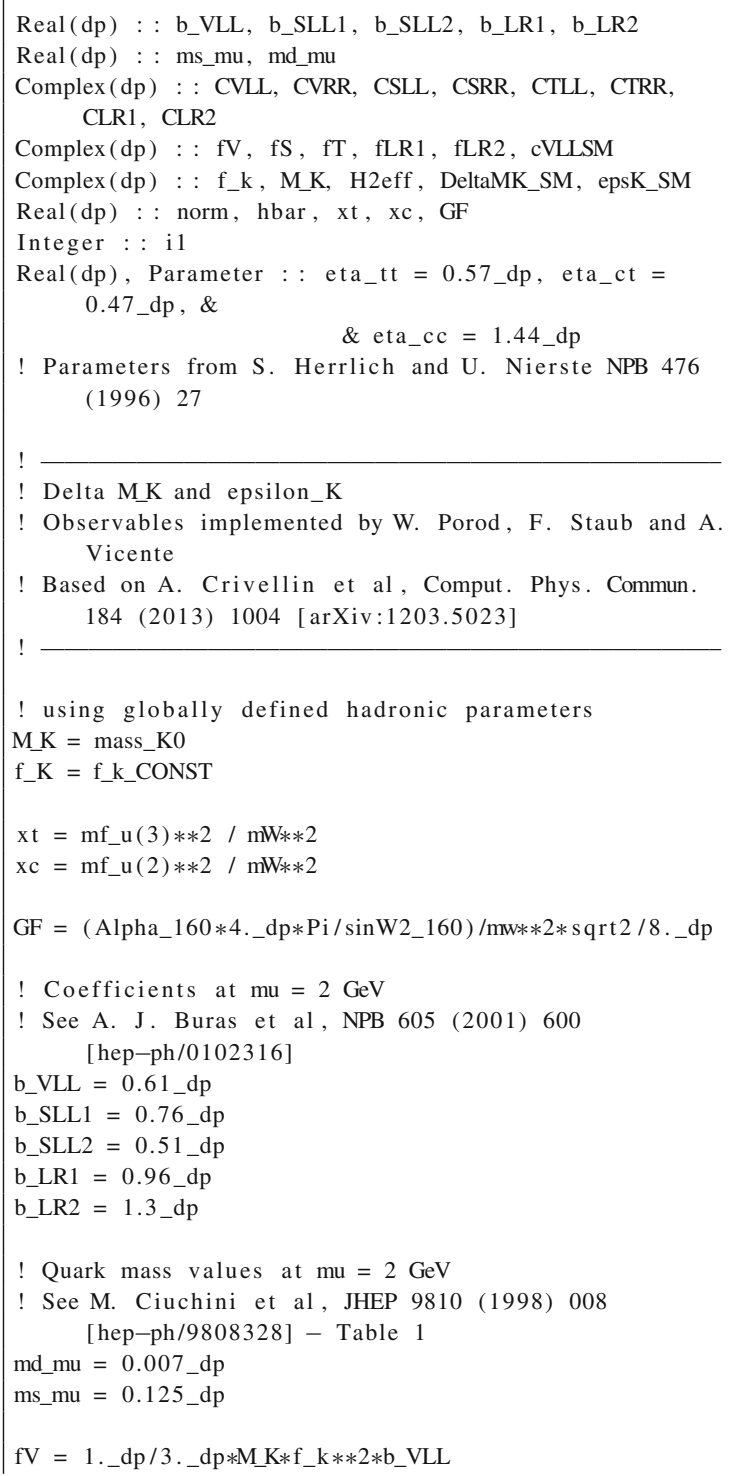




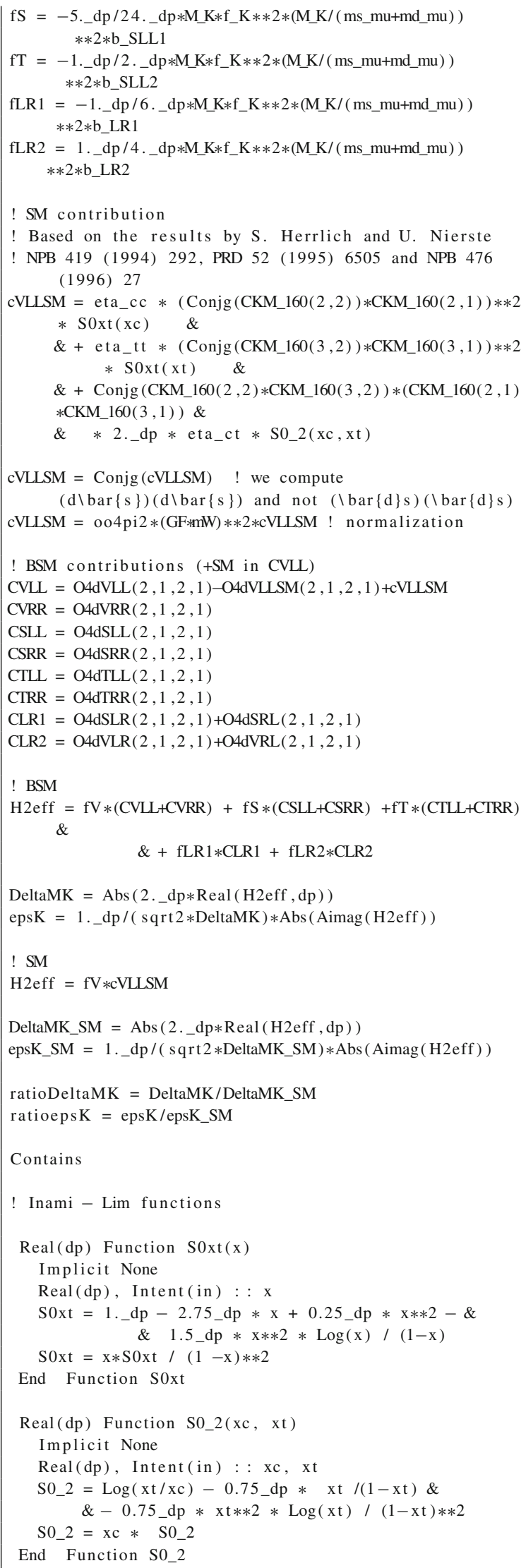

\section{C.11P $\rightarrow \ell v$}

Although $P \rightarrow \ell v$, where $P=q q^{\prime}$ is a pseudoscalar meson, does not violate quark flavor, we have included it in the list of observables for practical reasons, as it can be computed with the same ingredients as the QFV observables. The decay width for the process $P \rightarrow \ell_{\alpha} \nu$ is given by [139]

$$
\begin{aligned}
\Gamma\left(P \rightarrow \ell_{\alpha} \nu\right)= & \frac{\left|G_{F} f_{P}\left(m_{P}^{2}-m_{\ell_{\alpha}}^{2}\right)\right|^{2}}{8 \pi m_{P}^{3}} \\
& \times \sum_{v} \mid V_{q q^{\prime} m_{\ell_{\alpha}}}+\frac{m_{\ell_{\alpha}}}{2 \sqrt{2}}\left(G_{L L}^{V}-G_{R L}^{V}\right) \\
& +\left.\frac{m_{P}^{2}}{2 \sqrt{2}\left(m_{q}+m_{q^{\prime}}\right)}\left(G_{R R}^{S}-G_{L R}^{S}\right)\right|^{2} .
\end{aligned}
$$

Here $f_{P}$ is the meson decay constant, $m_{q}$ and $m_{q^{\prime}}$ are the masses of the quarks in the meson and the Wilson coefficients $G_{X Y}^{I}$ are defined in Eq. (A.16). The sum in Eq. (C.102) is over the three neutrinos (whose masses are neglected).

Each $P \rightarrow \ell_{\alpha} \nu$ decay width is plagued by hadronic uncertainties. However, by taking the ratios

$R_{P}=\frac{\Gamma(P \rightarrow e \nu)}{\Gamma(P \rightarrow \mu \nu)}$

the hadronic uncertainties cancel out to a good approximation, allowing for a precise theoretical determination. In case of $R_{K}$, the SM prediction includes small electromagnetic corrections that account for internal bremsstrahlung and structure-dependent effects [140]. This leads to an impressive theoretical uncertainty of $\delta R_{K} / R_{K} \sim 0.1 \%$, making $R_{P}$ the perfect observable to search for lepton flavor universality violation [141].

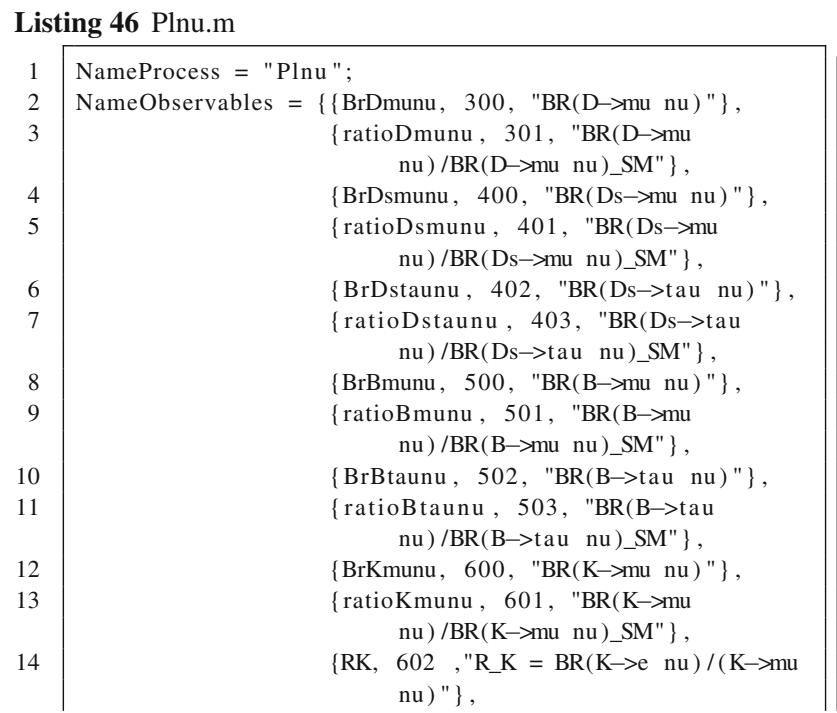




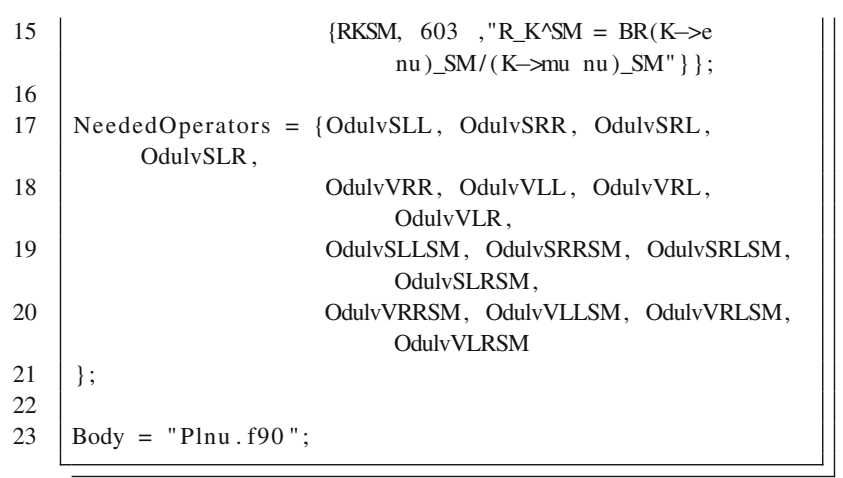

\section{Listing 47 Plnu.f90}

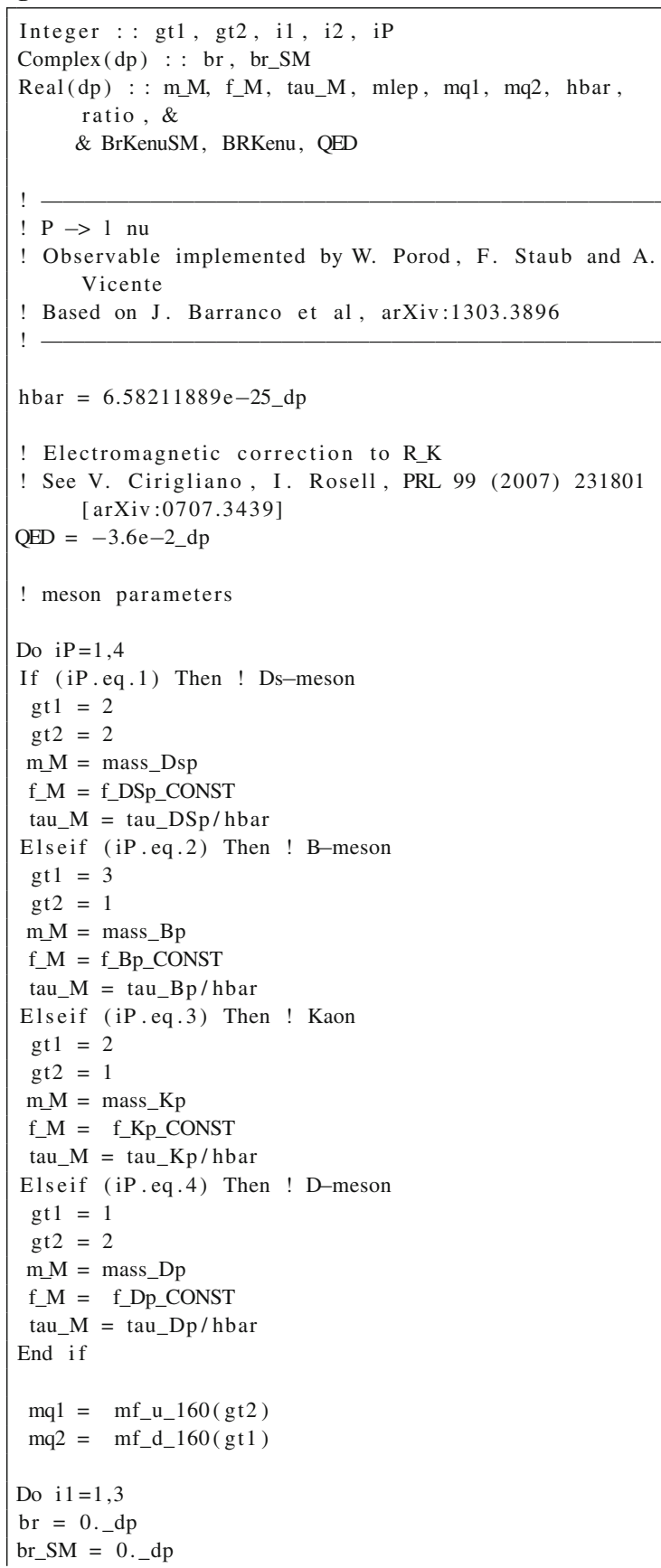

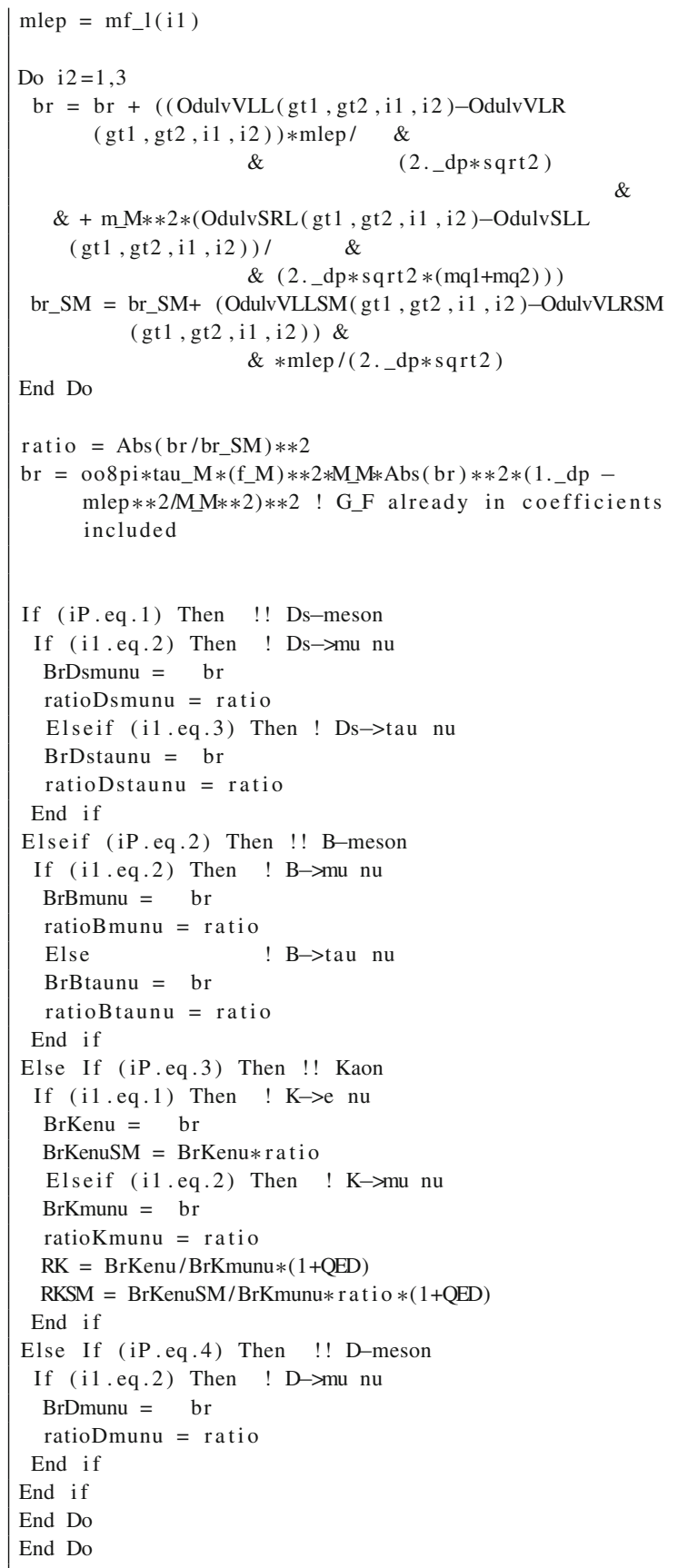

\section{Appendix D: Models}

The following models are included in the public version of SARAH and can now be used together with the Flavorkit to get predictions for the different observables.

\section{D.1 Supersymmetric models}

- Minimal supersymmetric standard model (see Ref. [142] and references therein) 
- With general flavor and CP structure (MSSM)

- Without flavor violation (MSSM/NoFV)

- With explicit CP violation in the Higgs sector (MSSM/CPV)

- In SCKM basis (MSSM/CKM)

- Singlet extensions:

- Next-to-minimal supersymmetric standard model (NMSSM, NMSSM/NoFV, NMSSM/CPV, NMSSM/ $\mathrm{CKM}$ ) (see Refs. [143,144] and references therein)

- near-to-minimal supersymmetric standard model (near-MSSM) [145]

- General singlet extended, supersymmetric standard model (SMSSM) [145, 146]

- DiracNMSSM (DiracNMSSM) [147, 148]

- Triplet extensions

- Triplet extended MSSM (TMSSM) [149]

- Triplet extended NMSSM (TNMSSM) [150]

- Models with $R$-parity violation [151-158]

- bilinear RpV (MSSM-RpV/Bi)

- Lepton number violation (MSSM-RpV/LnV)

- Only trilinear lepton number violation (MSSMRpV/TriLnV)

- Baryon number violation (MSSM-RpV/BnV)

- $\mu \nu \mathrm{SSM}$ (munuSSM) [159,160]

- Additional $U(1)^{\prime} s$

- U(1)-extended MSSM (UMSSM) [145]

- secluded MSSM (secluded-MSSM) [161]

- minimal $B-L$ model (B-L-SSM) [162-165]

- minimal singlet-extended $B-L$ model (N-B-LSSM)

- SUSY-scale seesaw extensions

- inverse seesaw (inverse-Seesaw) [166,167]

- linear seesaw (LinSeesaw) [166,168]

- singlet extended inverse seesaw (inverseSeesaw-NMSSM) [169]

- inverse seesaw with $B-L$ gauge group (B-L-SSMIS) [170]

- minimal $U(1)_{R} \times U(1)_{B-L}$ model with inverse seesaw (BLRinvSeesaw) [74,171]

- Models with Dirac Gauginos

- MSSM/NMSSM with Dirac Gauginos (DiracGauginos) [172-174]

- minimal $R$-Symmetric SSM (MRSSM) [175]

- Minimal Dirac Gaugino supersymmetric standard model (MDGSSM) [86]

- High-scale extensions
- Seesaw 1 -3 (SU(5) version), (Seesaw1,Seesaw2, Seesaw3) [63,65,68,176,177]

- Left/right model ( $\Omega$ LR) (Omega) [178, 179]

- Quiver model (QEW12, QEWm1 d2L3) [180]

D.2 Non-supersymmetric models

- Standard Model (SM) (SM), Standard model in CKM basis (SM/CKM) (see for instance Ref. [181] and references therein)

- inert Higgs doublet model (Inert) [182]

- B-L extended SM (B-L-SM) [183-185]

- B-L extended SM with inverse seesaw (B-L-SM-IS) [186]

- SM extended by a scalar color octet (SM-8C) [187]

- Two Higgs doublet model (THDM) (see for instance Ref. [188] and references therein)

- Singlet extended SM (SSM) [189]

- Singlet Scalar DM (SSDM) [190]

\section{References}

1. ATLAS Collaboration, G. Aad et al., Phys. Lett. B 716 (2012), 1-29. arXiv:1207.7214

2. CMS Collaboration, S. Chatrchyan et al., Phys. Lett. B 716, 30-61 (2012). arXiv: 1207.7235

3. ATLAS Collaboration, CDF Collaboration, CMS Collaboration, D0 Collaboration (2014). arXiv: 1403.4427

4. D. Buttazzo, G. Degrassi, P.P. Giardino, G.F. Giudice, F. Sala et al., JHEP 1312, 089 (2013). arXiv:1307.3536

5. F. Mahmoudi, Comput. Phys. Commun. 178, 745-754 (2008). arXiv:0710.2067

6. F. Mahmoudi, Comput. Phys. Commun. 180, 1579-1613 (2009). arXiv:0808.3144

7. F. Mahmoudi, Comput. Phys. Commun. 180, 1718-1719 (2009)

8. J. Rosiek, P. Chankowski, A. Dedes, S. Jager, P. Tanedo, Comput. Phys. Commun. 181, 2180-2205 (2010). arXiv: 1003.4260

9. A. Crivellin, J. Rosiek, P. Chankowski, A. Dedes, S. Jaeger et al., Comput. Phys. Commun. 184, 1004-1032 (2013). arXiv: 1203.5023

10. U. Ellwanger, C. Hugonie, Comput. Phys. Commun. 177, 399407 (2007). hep-ph/0612134

11. G. Belanger, F. Boudjema, A. Pukhov, A. Semenov, Comput. Phys. Commun. 149, 103-120 (2002). hep-ph/0112278

12. G. Belanger, F. Boudjema, A. Pukhov, A. Semenov, Comput. Phys. Commun. 174, 577-604 (2006). hep-ph/0405253

13. G. Belanger, F. Boudjema, A. Pukhov, A. Semenov, Comput. Phys. Commun. 176, 367-382 (2007). hep-ph/0607059

14. G. Belanger, F. Boudjema, A. Pukhov, A. Semenov, Comput. Phys. Commun. 180, 747-767 (2009). arXiv:0803.2360

15. G. Belanger, F. Boudjema, A. Pukhov, A. Semenov, Comput. Phys. Commun. 185, 960-985 (2014). arXiv:1305.0237

16. G. Degrassi, P. Gambino, P. Slavich, Comput. Phys. Commun. 179, 759-771 (2008). arXiv:0712.3265

17. B. Murakami, Comput. Phys. Commun. 185, 622-637 (2014). arXiv: 1302.4469

18. D. Chowdhury, R. Garani, S.K. Vempati, Comput. Phys. Commun. 184, 899-918 (2013). arXiv:1109.3551 
19. F.E. Paige, S.D. Protopopescu, H. Baer, X. Tata (2003) hep-ph/0312045

20. H. Baer, C. Balazs, A. Belyaev, R. Dermisek, A. Mafi et al., Nucl. Instrum. Methods A A502, 560-563 (2003)

21. H. Baer, F.E. Paige, S.D. Protopopescu, X. Tata (1999). hep-ph/0001086

22. F.E. Paige, S.D. Protopescu, H. Baer, X. Tata (1998). hep-ph/9810440

23. F.E. Paige, S.D. Protopopescu, H. Baer, X. Tata (1998). hep-ph/9804321

24. H. Baer, F.E. Paige, S.D. Protopopescu, X. Tata (1993). hep-ph/9305342

25. W. Porod, Comput. Phys. Commun. 153, 275-315 (2003). hep-ph/0301101

26. W. Porod, F. Staub, Comput. Phys. Commun. 183, 2458-2469 (2012). arXiv:1104.1573

27. F. Staub (2008). arXiv:0806.0538

28. F. Staub, Comput. Phys. Commun. 181, 1077-1086 (2010). arXiv:0909.2863

29. F. Staub, Comput. Phys. Commun. 182, 808-833 (2011). arXiv: 1002.0840

30. F. Staub, Comput. Phys. Commun. 184, 1792-1809 (2013). arXiv: 1207.0906

31. F. Staub, Comput. Phys. Commun. 185, 1773-1790 (2014). arXiv: 1309.7223

32. H. Dreiner, K. Nickel, F. Staub, A. Vicente, Phys. Rev. D 86, 015003 (2012). arXiv: 1204.5925

33. H. Dreiner, K. Nickel, W. Porod, F. Staub, Comput. Phys. Commun. 184, 2604-2617 (2013). arXiv:1212.5074

34. H. Dreiner, K. Nickel, F. Staub, Phys. Rev. D 88, 115001 (2013). arXiv: 1309.1735

35. T. Hahn, M. Perez-Victoria, Comput. Phys. Commun. 118, 153165 (1999). hep-ph/9807565

36. T. Hahn, Comput. Phys. Commun. 140, 418-431 (2001). hep-ph/0012260

37. T. Hahn, Nucl. Phys. Proc. Suppl. 89, 231-236 (2000). hep-ph/0005029

38. T. Hahn, Nucl. Phys. Proc. Suppl. 135, 333-337 (2004). hep-ph/0406288

39. T. Hahn, eConf C 050318, 0604 (2005). hep-ph/0506201

40. B.C. Nejad, T. Hahn, J.N. Lang, E. Mirabella, J. Phys. Conf. Ser. 523, 012050 (2014). doi:10.1088/1742-6596/523/1/012050. arXiv: 1310.0274

41. E. Ma, A. Pramudita, Phys. Rev. D 24, 1410 (1981)

42. J. Hisano, T. Moroi, K. Tobe, M. Yamaguchi, Phys. Rev. D 53, 2442-2459 (1996). hep-ph/9510309

43. A. Bednyakov, S. Tanyildizi (2013). arXiv: 1311.5546

44. T. Ohl, Comput. Phys. Commun. 90, 340-354 (1995). hep-ph/9505351

45. F. Staub, T. Ohl, W. Porod, C. Speckner, Comput. Phys. Commun. 183, 2165-2206 (2012). arXiv: 1109.5147

46. A. Crivellin, L. Hofer, J. Rosiek, JHEP 1107, 017 (2011). arXiv: 1103.4272

47. A.J. Buras, M. Jamin, P.H. Weisz, Nucl. Phys. B 347, 491-536 (1990)

48. G. Buchalla, A.J. Buras, Nucl. Phys. B 412, 106-142 (1994). hep-ph/9308272

49. M. Ciuchini, E. Franco, V. Gimenez, Phys. Lett. B 388, 167-172 (1996). hep-ph/9608204

50. A.J. Buras, P. Gambino, U.A. Haisch, Nucl. Phys. B 570, 117-154 (2000). hep-ph/9911250

51. M. Misiak, M. Steinhauser, Nucl. Phys. B 764, 62-82 (2007). hep-ph/0609241

52. A.J. Buras, A. Czarnecki, M. Misiak, J. Urban, Nucl. Phys. B 631, 219-238 (2002). hep-ph/0203135
53. R. Boughezal, M. Czakon, T. Schutzmeier, JHEP 0709, 072 (2007). arXiv:0707.3090

54. A.J. Buras, J. Girrbach, JHEP 1203, 052 (2012). arXiv:1201.1302

55. F. Mahmoudi, S. Heinemeyer, A. Arbey, A. Bharucha, T. Goto et al., Comput. Phys. Commun. 183, 285-298 (2012). arXiv: 1008.0762

56. A. Ilakovac, A. Pilaftsis, Nucl. Phys. B 437, 491 (1995). hep-ph/9403398

57. A. Ilakovac, A. Pilaftsis, L. Popov, Phys. Rev. D 87(5), 053014 (2013). arXiv:1212.5939

58. E. Arganda, M.J. Herrero, Phys. Rev. D 73, 055003 (2006). hep-ph/0510405

59. F. Deppisch, H. Pas, A. Redelbach, R. Ruckl, Y. Shimizu, Eur Phys. J. C 28, 365-374 (2003). hep-ph/0206122

60. S. Petcov, T. Shindou, Y. Takanishi, Nucl. Phys. B 738, 219-242 (2006). hep-ph/0508243

61. S. Antusch, E. Arganda, M. Herrero, A. Teixeira, JHEP 0611, 090 (2006). hep-ph/0607263

62. P. Paradisi, JHEP 0608, 047 (2006). hep-ph/0601100

63. M. Hirsch, J. Valle, W. Porod, J. Romao, A. Villanova del Moral, Phys. Rev. D 78, 013006 (2008). arXiv:0804.4072

64. M. Hirsch, S. Kaneko, W. Porod, Phys. Rev. D 78, 093004 (2008). arXiv:0806.3361

65. F. Borzumati, T. Yamashita, Prog. Theor. Phys. 124, 761-868 (2010). arXiv:0903.2793

66. E. Gross, D. Grossman, Y. Nir, O. Vitells, Phys. Rev. D 81, 055013 (2010). arXiv:1001.2883

67. C. Biggio, L. Calibbi, JHEP 1010, 037 (2010). arXiv:1007.3750

68. J. Esteves, J. Romao, M. Hirsch, F. Staub, W. Porod, Phys. Rev. D 83, 013003 (2011). arXiv: 1010.6000

69. A. Abada, A. Figueiredo, J. Romao, A. Teixeira, JHEP 1010, 104 (2010). arXiv:1007.4833

70. A. Abada, A. Figueiredo, J. Romao, A. Teixeira, JHEP 1108, 099 (2011). arXiv:1104.3962

71. C.G. Cely, A. Ibarra, E. Molinaro, S. Petcov, Phys. Lett. B 718 957-964 (2013). arXiv:1208.3654

72. M. Hirsch, W. Porod, C. Weiss, F. Staub, Phys. Rev. D 87, 013010 (2013). arXiv:1211.0289

73. S. Davidson, P. Verdier, Phys. Rev. D 86, 111701 (2012). arXiv: 1211.1248

74. M. Hirsch, W. Porod, L. Reichert, F. Staub, Phys. Rev. D 86 , 093018 (2012). arXiv:1206.3516

75. D. Dinh, A. Ibarra, E. Molinaro, S. Petcov, JHEP 1208, 125 (2012). arXiv:1205.4671

76. M. Cannoni, J. Ellis, M.E. Gomez, S. Lola, Phys. Rev. D 88 , 075005 (2013). arXiv:1301.6002

77. M.E. Krauss, W. Porod, F. Staub, A. Abada, A. Vicente et al., Phys. Rev. D 90, 013008 (2014). doi:10.1103/PhysRevD.90.013008. arXiv: 1312.5318

78. M. Arana-Catania, E. Arganda, M. Herrero, JHEP 1309, 160 (2013). arXiv:1304.3371

79. W. Altmannshofer, M. Bauer, M. Carena, JHEP 1401, 060 (2014). arXiv: 1308.1987

80. A. Crivellin, S. Najjari, J. Rosiek, JHEP 1404, 167 (2014) arXiv: 1312.0634

81. A. Celis, V. Cirigliano, E. Passemar, Phys. Rev. D 89, 013008 (2014). arXiv:1309.3564

82. T. Moroi, M. Nagai, T.T. Yanagida, Phys. Lett. B 728, 342-346 (2014). arXiv:1305.7357

83. D. Dinh, S. Petcov, JHEP 1309, 086 (2013). arXiv:1308.4311

84. A. Falkowski, D.M. Straub, A. Vicente, JHEP 1405, 092 (2014). doi:10.1007/JHEP05(2014)092. arXiv:1312.5329

85. T. Toma, A. Vicente, JHEP 1401, 160 (2014). arXiv:1312.2840

86. K. Benakli, M. Goodsell, F. Staub, W. Porod (2014). arXiv: 1403.5122 
87. A. Teixeira, A. Abada, A. Figueiredo, J. Romao (2014). arXiv: 1402.1426

88. A. Celis, V. Cirigliano, E. Passemar, Phys. Rev. D 89, 095014 (2014). doi:10.1103/PhysRevD.89.095014. arXiv:1403.5781

89. A. Crivellin, M. Hoferichter, M. Procura, Phys. Rev. D 89, 093024 (2014). doi:10.1103/PhysRevD.89.093024. arXiv:1404. 7134

90. Y. Kuno, Y. Okada, Rev. Mod. Phys. 73, 151-202 (2001). hep-ph/9909265

91. E. Arganda, M. Herrero, A. Teixeira, JHEP 0710, 104 (2007). arXiv:0707.2955

92. H. Chiang, E. Oset, T. Kosmas, A. Faessler, J. Vergados, Nucl. Phys. A 559, 526-542 (1993)

93. T. Kosmas, S. Kovalenko, I. Schmidt, Phys. Lett. B 511, 203 (2001). hep-ph/0102101

94. E. Arganda, M. Herrero, J. Portoles, JHEP 0806, 079 (2008). arXiv:0803.2039

95. P. Paradisi, JHEP 0602, 050 (2006). hep-ph/0508054

96. E. Arganda, A.M. Curiel, M.J. Herrero, D. Temes, Phys. Rev. D 71, 035011 (2005). hep-ph/0407302

97. X.-J. Bi, Y.-B. Dai, X.-Y. Qi, Phys. Rev. D 63, 096008 (2001). hep-ph/0010270

98. M. Ciuchini, V. Lubicz, L. Conti, A. Vladikas, A. Donini et al., JHEP 9810, 008 (1998). hep-ph/9808328

99. G. Buchalla, A.J. Buras, Nucl. Phys. B 400, 225-239 (1993)

100. M. Misiak, J. Urban, Phys. Lett. B 451, 161-169 (1999). hep-ph/9901278

101. A. Dedes, H.K. Dreiner, U. Nierste, Phys. Rev. Lett. 87, 251804 (2001). hep-ph/0108037

102. W. Altmannshofer, D.M. Straub, Eur. Phys. J. C 73, 2646 (2013). arXiv: 1308.1501

103. A. Dedes, J. Rosiek, P. Tanedo, Phys. Rev. D 79, 055006 (2009). arXiv:0812.4320

104. E. Lunghi, J. Matias, JHEP 0704, 058 (2007). hep-ph/0612166

105. C. Bobeth, A.J. Buras, F. Kruger, J. Urban, Nucl. Phys. B 630, 87-131 (2002). hep-ph/0112305

106. T. Huber, E. Lunghi, M. Misiak, D. Wyler, Nucl. Phys. B 740, 105-137 (2006). hep-ph/0512066

107. H.E. Logan, U. Nierste, Nucl. Phys. B 586, 39-55 (2000). hep-ph/0004139

108. A.J. Buras, P.H. Chankowski, J. Rosiek, L. Slawianowska, Nucl. Phys. B 659, 3 (2003). hep-ph/0210145

109. W.-S. Hou, Phys. Rev. D 48, 2342-2344 (1993)

110. T. Ibrahim, P. Nath, Phys. Rev. D 61, 095008 (2000). hep-ph/9907555

111. R. Barbieri, D. Buttazzo, F. Sala, D.M. Straub, A. Tesi, JHEP 1305, 069 (2013), arXiv:1211.5085

112. W. Altmannshofer, D.M. Straub, JHEP 1208, 121 (2012). arXiv: 1206.0273

113. D. Becirevic, N. Kosnik, F. Mescia, E. Schneider, Phys. Rev. D 86, 034034 (2012). arXiv:1205.5811

114. D. Becirevic, N. Kosnik, A. Tayduganov, Phys. Lett. B 716, 208 213 (2012). arXiv: 1206.4977

115. A.J. Buras, J. Girrbach (2013). arXiv: 1306.3775

116. I. Dorsner, S. Fajfer, N. Kosnik, I. Nisandzic, JHEP 1311, 084 (2013). arXiv:1306.6493

117. S. Descotes-Genon, J. Matias, J. Virto, Phys. Rev. D 88, 074002 (2013). arXiv: 1307.5683

118. A.J. Buras, F. De Fazio, J. Girrbach, JHEP 1402, 112 (2014). arXiv: 1311.6729

119. R. Barbieri, D. Buttazzo, F. Sala, D.M. Straub, JHEP 1405, 105 (2014). doi:10.1007/JHEP05(2014)105. arXiv:1402.6677

120. A.J. Buras, F. De Fazio, J. Girrbach (2014). arXiv:1404.3824

121. M. König, M. Neubert, D.M. Straub (2014). arXiv:1403.2756

122. A. Greljo, J.F. Kamenik, J. Kopp, JHEP 1407, 046 (2014). doi:10. 1007/JHEP07(2014)046. arxiv:1404.1278
123. C. Bobeth, T. Ewerth, F. Kruger, J. Urban, Phys. Rev. D 66, 074021 (2002). hep-ph/0204225

124. G. Isidori, A. Retico, JHEP 0209, 063 (2002). hep-ph/0208159

125. A.L. Kagan, M. Neubert, Eur. Phys. J. C 7, 5-27 (1999). hep-ph/9805303

126. M. Misiak, H. Asatrian, K. Bieri, M. Czakon, A. Czarnecki et al., Phys. Rev. Lett. 98, 022002 (2007). hep-ph/0609232

127. E. Lunghi, Private communication

128. Particle Data Group, J. Beringer et al., Phys. Rev. D 86, 010001 (2012)

129. G. Buchalla, A.J. Buras, Nucl. Phys. B 548, 309-327 (1999). hep-ph/9901288

130. A.J. Buras, P.H. Chankowski, J. Rosiek, L. Slawianowska, Nucl. Phys. B 619, 434-466 (2001). hep-ph/0107048

131. J. Urban, F. Krauss, U. Jentschura, G. Soff, Nucl. Phys. B 523, 40-58 (1998). hep-ph/9710245

132. J. Laiho, E. Lunghi, R.S. Van de Water, Phys. Rev. D 81, 034503 (2010). arxiv:0910.2928

133. T. Inami, C. Lim, Prog. Theor. Phys. 65, 297 (1981)

134. A.J. Buras, 281-539 (1998). hep-ph/9806471

135. A.J. Buras, S. Jager, J. Urban, Nucl. Phys. B 605, 600-624 (2001). hep-ph/0102316

136. S. Herrlich, U. Nierste, Nucl. Phys. B 419, 292-322 (1994). hep-ph/9310311

137. S. Herrlich, U. Nierste, Phys. Rev. D 52, 6505-6518 (1995). hep-ph/9507262

138. S. Herrlich, U. Nierste, Nucl. Phys. B 476, $27-88$ (1996). hep-ph/9604330

139. J. Barranco, D. Delepine, V. Gonzalez Macias, L. Lopez-Lozano, Phys. Lett. B 731, 36-42 (2014). arxiv:1303.3896

140. V. Cirigliano, I. Rosell, Phys. Rev. Lett. 99, 231801 (2007). arxiv:0707.3439

141. A. Abada, D. Das, A. Teixeira, A. Vicente, C. Weiland, JHEP 1302, 048 (2013). arxiv: 1211.3052

142. S.P. Martin (1997). hep-ph/9709356

143. M. Maniatis, Int. J. Mod. Phys. A 25, 3505-3602 (2010). arxiv:0906.0777

144. U. Ellwanger, C. Hugonie, A.M. Teixeira, Phys. Rep. 496, 1-77 (2010). arxiv:0910.1785

145. V. Barger, P. Langacker, H.-S. Lee, G. Shaughnessy, Phys. Rev. D 73, 115010 (2006). hep-ph/0603247

146. G.G. Ross, K. Schmidt-Hoberg, F. Staub, JHEP 1208, 074 (2012). arxiv: 1205.1509

147. X. Lu, H. Murayama, J.T. Ruderman, K. Tobioka, Phys. Rev. Lett. 112, 191803 (2014). doi:10.1103/PhysRevLett.112.191803. arxiv: 1308.0792

148. A. Kaminska, G.G. Ross, K. Schmidt-Hoberg, F. Staub (2014). arxiv: 1401.1816

149. S. Di Chiara, K. Hsieh, Phys. Rev. D 78, 055016 (2008), arxiv:0805.2623

150. K. Agashe, A. Azatov, A. Katz, D. Kim, Phys. Rev. D 84, 115024 (2011). arxiv:1109.2842

151. L.J. Hall, M. Suzuki, Nucl. Phys. B 231, 419 (1984)

152. H.K. Dreiner (1997). hep-ph/9707435

153. B. Allanach, A. Dedes, H. Dreiner, Phys. Rev. D 69, 115002 (2004). hep-ph/0309196

154. G. Bhattacharyya (1997). hep-ph/9709395

155. V.D. Barger, G. Giudice, T. Han, Phys. Rev. D 40, 2987 (1989)

156. B. Allanach, A. Dedes, H.K. Dreiner, Phys. Rev. D 60, 075014 (1999). hep-ph/9906209

157. M. Hirsch, M. Diaz, W. Porod, J. Romao, J. Valle, Phys. Rev. D 62, 113008 (2000). hep-ph/0004115

158. R. Barbier, C. Berat, M. Besancon, M. Chemtob, A. Deandrea et al., Phys. Rept. 420, 1-202 (2005). hep-ph/0406039

159. D. Lopez-Fogliani, C. Munoz, Phys. Rev. Lett. 97, 041801 (2006). hep-ph/0508297 
160. A. Bartl, M. Hirsch, A. Vicente, S. Liebler, W. Porod, JHEP 0905 , 120 (2009). arxiv:0903.3596

161. C.-W. Chiang, E. Senaha, JHEP 1006, 030 (2010). arxiv:0912.5069

162. S. Khalil, A. Masiero, Phys. Lett. B 665, 374-377 (2008). arxiv:0710.3525

163. P. Fileviez Perez, S. Spinner, Phys. Rev. D 83, 035004 (2011). arxiv: 1005.4930

164. B. O'Leary, W. Porod, F. Staub, JHEP 1205, 042 (2012). arxiv: 1112.4600

165. L. Basso, B. O'Leary, W. Porod, F. Staub, JHEP 1209, 054 (2012). arxiv:1207.0507

166. M. Malinsky, J. Romao, J. Valle, Phys. Rev. Lett. 95, 161801 (2005). hep-ph/0506296

167. P. Bhupal Dev, S. Mondal, B. Mukhopadhyaya, S. Roy, JHEP 1209, 110 (2012). arxiv: 1207.6542

168. V. De Romeri, M. Hirsch, JHEP 1212, 106 (2012). arxiv: 1209.3891

169. I. Gogoladze, B. He, Q. Shafi, Phys. Lett. B 718, 1008-1013 (2013). arxiv:1209.5984

170. L. Basso, A. Belyaev, D. Chowdhury, M. Hirsch, S. Khalil et al., Comput. Phys. Commun. 184, 698-719 (2013). arxiv:1206.4563

171. M. Hirsch, M. Malinsky, W. Porod, L. Reichert, F. Staub, JHEP 1202, 084 (2012). arxiv: 1110.3037

172. G. Belanger, K. Benakli, M. Goodsell, C. Moura, A. Pukhov, JCAP 0908, 027 (2009). arxiv:0905.1043

173. K. Benakli, M. Goodsell, Nucl. Phys. B 840, 1-28 (2010). arxiv: 1003.4957

174. K. Benakli, M.D. Goodsell, F. Staub, JHEP 1306, 073 (2013). arxiv: 1211.0552
175. G.D. Kribs, E. Poppitz, N. Weiner, Phys. Rev. D 78, 055010 (2008). arxiv:0712.2039

176. A. Rossi, Phys. Rev. D 66, 075003 (2002). hep-ph/0207006

177. J. Esteves, J. Romao, A. Villanova del Moral, M. Hirsch, J. Valle et al., JHEP 0905, 003 (2009). arxiv:0903.1408

178. J. Esteves, J. Romao, M. Hirsch, A. Vicente, W. Porod et al., JHEP 1012, 077 (2010). arxiv: 1011.0348

179. J. Esteves, J. Romao, M. Hirsch, W. Porod, F. Staub et al., JHEP 1201, 095 (2012). arxiv: 1109.6478

180. A. Bharucha, A. Goudelis, M. McGarrie, Eur. Phys. J. C 74, 2858 (2014). doi:10.1140/epjc/s10052-014-2858-7. arxiv: 1310.4500

181. W. Hollik (2010). arxiv: 1012.3883

182. L. Lopez Honorez, E. Nezri, J.F. Oliver, M.H. Tytgat, JCAP 0702, 028 (2007). hep-ph/0612275

183. W. Emam, S. Khalil, Eur. Phys. J. C 52, 625-633 (2007). arxiv:0704.1395

184. L. Basso, A. Belyaev, S. Moretti, C.H. Shepherd-Themistocleous, Phys. Rev. D 80, 055030 (2009). arxiv:0812.4313

185. L. Basso, A. Belyaev, S. Moretti, G.M. Pruna, JHEP 0910, 006 (2009). arxiv:0903.4777

186. S. Khalil, Phys. Rev. D 82, 077702 (2010). arxiv:1004.0013

187. H.H. Patel, M.J. Ramsey-Musolf, M.B. Wise, Phys. Rev. D 88(1), 015003 (2013). arxiv:1303.1140

188. G. Branco, P. Ferreira, L. Lavoura, M. Rebelo, M. Sher et al., Phys. Rep. 516, 1-102 (2012). arxiv: 1106.0034

189. D. O’Connell, M.J. Ramsey-Musolf, M.B. Wise, Phys. Rev. D 75, 037701 (2007). hep-ph/0611014

190. A. Goudelis, Y. Mambrini, C. Yaguna, JCAP 0912, 008 (2009). arxiv:0909.2799 